\title{
Valores vazios e incerteza no modelo de dados relacional
}

\section{Maristella Ribas Biazzi}

\author{
DISSERTAÇÃO APRESENTADA \\ $\mathrm{AO}$ \\ INSTITUTO DE MATEMÁTICA E ESTATÍSTICA \\ DA \\ UNIVERSIDADE DE SÃO PAULO \\ PARA OBTENÇÃO DO GRAU DE MESTRE \\ $\mathrm{EM}$ \\ MATEMÁTICA APLICADA
}

Área de Concentração: Ciência da Computação

Orientador: Prof. Dr. Valdemar W. Setzer

-São Paulo, Novembro de 1993- 


\section{Valores vazios e incerteza no modelo de dados relacional}

Este exemplar corresponde à redação final da dissertação devidamente corrigida e defendida por Maristella Ribas Biazzi e aprovada pela comissão julgadora.

São Paulo, 25 de novembro de 1993.

Banca examinadora:

- Prof. Dr. Valdemar W. Setzer (orientador) - IME-USP

- Prof. Dr. Flávio Soares Corrêa da Silva - IME-USP

- Prof. Dr. Marco Antonio Casanova - IBM-RJ 
aos meus pais

Hélio e Fernanda

e ao meu querido

Mário 


\section{Agradecimentos}

Ao Prof. Dr. Valdemar W. Setzer pela orientação, acompanhamento, incentivo e dedicação, mesmo quando não poderia e não deveria se preocupar com o trabalho. Não há palavras ou gestos que possam expressar a intensidade de minha gratidão.

Ao amigo Flavio, pelo incentivo e pelas interessantes discussões sobre o assunto.

À minha família, em particular minhas irmãs Mônica e Maria Fernanda, minha tia Márcia, minha sobrinha Natália, meus avós Iracema e Natalio, por toda uma vida de amizade, amor, e incentivo em todos os meus projetos pessoais.

Aos meus sogros Mário e Mariza, pelo acompanhamento e incentivo durante a elaboração do trabalho.

Aos amigos da TECHNE, pelo apoio e incentivo que possibilitaram a elaboração deste trabalho.

Aos funcionários e professores do Instituto, pela compreensão e apoio em todos os momentos necessários.

Aos amigos que direta e indiretamente ajudaram e incentivaram na elaboração deste trabalho. 


\section{Resumo}

Este texto constitui, basicamente, uma resenha sobre o tratamento de valores vazios e informações incompletas no modelo de dados relacional. Apresentamos também uma proposta de implementação de um sistema gerenciador de bancos de dados que permite um tratamento correto de valores vazios.

A resenha abrange diversos aspectos do uso de valores vazios no modelo relacional, tais como formas de representação, consultas e atualizações, e interpretação de fatos negativos. Apresentamos também um histórico das pesquisas na área, que são relativamente recentes.

\section{Abstract}

This dissertation contitutes basically a survey on how to deal with null values and incomplete information in the relational data model. We also propose an implementation of a database management system that allows the correct use of null values.

The survey covers many aspects of the use of null values in the relational data model, such as representation, query and update processing, and the interpretation of negative facts. We also present a historical account of the research results in the field, which are relatively recent. 


\section{Conteúdo}

1 Introdução $\quad 5$

2 Definições básicas $\quad 9$

2.1 Modelo Relacional . . . . . . . . . . . . . . . . . . 9

2.2 Álgebra Relacional . . . . . . . . . . . . . . . . . 10

2.3 Dependências Funcionais e Restrições de Integridade . . . . . . . . . 11

$2.4 \mathrm{O}$ modelo relacional e a lógica . . . . . . . . . . . . . . . . 13

2.5 Hipóteses sobre Bancos de Dados: CWA e OWA . . . . . . . . . . . . 14

3 Valores vazios 16

3.1 Tipos de valores vazios . . . . . . . . . . . . . . . . 16

3.1 .1 O valor vazio aplicável . . . . . . . . . . . . . . 17

3.1 .2 O valor vazio inaplicável . . . . . . . . . . . . . 18

3.1.3 O valor vazio desconhecido . . . . . . . . . . . . . 19

3.2 Lógica de 3 valores . . . . . . . . . . . . . . . . . . . . . 21

3.3 Lógica de 4 valores . . . . . . . . . . . . . . . . . . . . . 23

3.4 Lógica de 7 valores e simplificações . . . . . . . . . . . . . . . . . 26

4 Representações algébricas $\quad 31$

4.1 Relações com valores vazios . . . . . . . . . . . . . . . . . . . . 31

4.2 Relação Universal . . . . . . . . . . . . . . . . . . . . . . . . . . . . 40

4.2 .1 Sistemas de informação . . . . . . . . . . . . . . 40 
4.2 .2 Instâncias universais legais . . . . . . . . . . . . . 44

4.3 Fundamentos de Representações Formais . . . . . . . . . . . . . 46

$4.4 \mathrm{~V}$-tabelas e C-tabelas . . . . . . . . . . . . . . 52

4.5 Relações com valores lógicos armazenados . . . . . . . . . . . 54

4.6 Informação Indefinida . . . . . . . . . . . . 55

$5 \quad$ Representações lógicas $\quad \mathbf{5 9}$

5.1 Linguagens Relacionais . . . . . . . . . . . . . . . . 60

5.2 Semântica de linguagens relacionais . . . . . . . . . . . . . 61

5.3 Teoria de prova . . . . . . . . . . . . . . . 65

5.4 Semântica de partição . . . . . . . . . . . . . . 69

6 Consultas $\quad 71$

6.1 Seleção e Junção . . . . . . . . . . . . . . . . . . . . . . 71

6.2 Propriedades de extensões da álgebra relacional . . . . . . . . . 72

6.3 Semântica de consultas com valores vazios . . . . . . . . . . . . . . . 74

6.4 Otimização de consultas com valores vazios . . . . . . . . . . 76

6.5 Consultas em representação lógica . . . . . . . . . . . . . . . . 77

6.6 Algoritmos de consulta em representação lógica . . . . . . . . . . . 79

6.7 Algoritmo I . . . . . . . . . . . . . . . . . . . . 79

6.8 Algoritmo II . . . . . . . . . . . . . . . . 86

7 Atualização $\quad 89$

7.1 Restrições de Integridade . . . . . . . . . . . . . . . . . 89

7.2 Atualização . . . . . . . . . . . . . . . . . . . . 92

7.2 .1 Inserção . . . . . . . . . . . . . . . . . . . 94

7.2 .2 Remoção . . . . . . . . . . . . . . . . . . . . 94

7.2 .3 Integração . . . . . . . . . . . . . . . . 95

7.2 .4 Sujeição . . . . . . . . . . . . . . . . . 95 
7.2 .5 Sujeição negativa . . . . . . . . . . . . . . . . . 9 96

7.2 .6 Adição . . . . . . . . . . . . . . . . . . . . . 97

7.2 .7 Modificação . . . . . . . . . . . . . . . . . . 97

7.3 Atualizações em representação lógica . . . . . . . . . . . . . . . 99

8 Negação 102

8.1 Hipótese do mundo fechado estendida . . . . . . . . . . . . . . 104

8.2 Valores universais e valores padrão . . . . . . . . . . . . . 106

8.3 Valores abertos . . . . . . . . . . . . . . . . . . 108

9 Tópicos especiais 111

9.1 O modelo relacional não normalizado $\ldots \ldots \ldots \ldots \ldots$

9.2 Bancos de dados orientados a objetos . . . . . . . . . . . 116

9.2 .1 Conceitos básicos . . . . . . . . . . . . . . . . 116

9.2 .2 Esquemas incompletos . . . . . . . . . . . . . . . 117

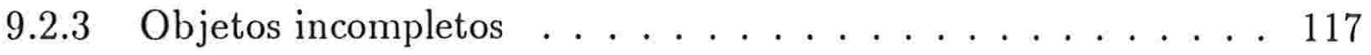

10 Proposta de implementação 119

10.1 Considerações Teóricas . . . . . . . . . . . . . . . . . . 120

10.1.1 Expressões de Comparação . . . . . . . . . . . . . . . . . . 120

10.1.2 Álgebra Relacional . . . . . . . . . . . . . . . . . 122

10.1 .3 Atualização . . . . . . . . . . . . . . . . . . . . . 124

10.2 Forma de implementação . . . . . . . . . . . . . . . . . . . . . . . 124

10.3 Indexação . . . . . . . . . . . . . . . . . . . . . 126

10.4 Linguagem de Acesso . . . . . . . . . . . . . . . . . . 127

10.5 Exemplos . . . . . . . . . . . . . . . . . . . . 129

10.5.1 Administração de Recursos Humanos . . . . . . . . . . . . 129

10.5 .2 Organograma . . . . . . . . . . . . . . . . . . . 129

10.5.3 Controle Acadêmico _. . . . . . . . . . . . . 130 
10.5.4 Requisições de Exames . . . . . . . . . . . . . . . . . 130

11 Conclusões

12 Bibliografia Comentada 


\section{Capítulo 1}

\section{Introdução}

Um banco de dados pretende ser uma ferramenta para representar fatos do mundo real de forma organizada e fiel à realidade. Frequentemente, porém, os dados sobre esta realidade são incompletos.

O objetivo deste texto é discorrer sobre alguns tipos comuns de informação incompleta em bancos de dados e a forma de os representar e tratar. Quando a informação é incompleta, temos dados que são parcial ou totalmente desconhecidos. Nesse caso, o local onde seria armazenada essa informação contém um valor vazio ou valor nulo.

O tratamento de informações incompletas em bancos de dados é um problema de ordem essencialmente prática. Um bom exemplo disso é o preenchimento de um formulário de dados pessoais. Existem inúmeros campos que, devido a falta de conhecimento dos dados no momento do preenchimento, não são preenchidos, ou que de fato não existem. Imagine o campo "CPF do cônjuge". Para uma pessoa casada, esta é uma informação que ela talvez não saiba. Por outro lado, para uma pessoa solteira, este dado simplesmente não existe.

Ainda no exemplo do formulário, suponha que exista um banco de dados com o cadastro de dados pessoais, e que o funcionário do setor alimente este banco através dos formulários preenchidos. Provavelmente este funcionário utilizará o seu próprio método de interpretar estes campos não preenchidos com frases do tipo "não há", ou simplesmente colocando espaços em branco ou zeros. Se, futuramente, for necessária uma lista dos cônjuges de funcionários contribuintes, por número do $\mathrm{CPF}$, este registro não aparecerá nesta lista.

Um outro exemplo onde aparecem informações incompletas é a criação de um novo campo no formulário, como "cartão de crédito internacional". Para os funcionários já cadastrados no banco, esse campo deve ser criado com valor "vazio", para posterior 
alteração quando a informação estiver disponível. No entanto, o fato desse campo estar vazio não significa que o funcionário não possua cartão de crédito internacional.

O gerenciador de banco de dados deve oferecer subsídios para a representação de informações incompletas. Tem-se pesquisado bastante para encontrar formas de representar essas informações. A maioria das pesquisas na área é feita utilizando o modelo relacional de bancos de dados, proposto por Codd em [Codd70] e que se tornou a base para quase todos os gerenciadores de bancos de dados comercializados hoje. O capítulo 2 apresenta as noções básicas desse modelo de representação de dados.

Entre os valores vazios, o relatório da ANSI/X3/SPARC [ANS75] relaciona 13 tipos diferentes:

1. Não válido para esta tupla. Exemplo: nota de exame de qualificação para aluno de graduação.

2. Válido, mas ainda não existe. Exemplo: nota de exame de qualificação para aluno que ainda não cumpriu os créditos mínimos.

3. Existe, mas a gravação não é autorizada. Exemplo: religião de um aluno.

4. Pode existir, mas é desconhecido. Exemplo: carta de habilitação para dirigir de um aluno cuja idade também é desconhecida.

5. Existe, mas ainda não foi gravado. Exemplo: nota de exame de qualificação para aluno cujo exame já foi corrigido, porém a nota não foi cadastrada.

6. Gravado e depois apagado.

7. Gravado mas ainda não disponível.

8. Disponível, mas em fase de mudança. Exemplo: saldo bancário no momento da compensação de cheques.

9. Disponível, mas não confiável. Exemplo: previsão da taxa de inflação mensal.

10. Disponível, mas inválido. Exemplo: saldo bancário em conta bloqueada ou suspensa.

11. Bloqueado para esta relação. Exemplo: salário de funcionários numa visão que não permite o acesso ao mesmo.

12. Bloqueado para esta tupla. Exemplo: salário de um funcionaário hierarquicamente superior. 
13. Bloqueado para este instante. Exemplo: saldo bancário com cheques que ainda serão compensados.

O próprio Codd chamou atenção para o problema de valores vazios em [Codd79] e [Codd87]. Codd usou a notação $\omega$ para o valor vazio, e introduziu a lógica de 3 valores (Falso, Verdadeiro, Desconhecido), para o tratamento de expressões que envolvem o valor vazio $\omega$. Segundo [Codd79], uma expressão pode ser falsa ou verdadeira, se as tuplas envolvidas na mesma não têm nenhuma ocorrência do valor vazio $\omega$, ou desconhecida caso contrário.

Diversos autores se propuseram a construir extensões do modelo relacional que representassem mais fielmente a realidade (p.ex. [Bis83], [Ges91], [Gra79], [LL86], [Yue91], [Zan84]). Nesse sentido, muitos criaram extensões da álgebra relacional para tabelas com valores vazios. [IL84] ressalta que o funcionamento dos operadores relacionais estendidos deve ser considerado em conjunto, isto é, não é suficiente a análise isolada do funcionamento dos mesmos pois pode-se encontrar problemas na composição de operadores diferentes.

O problema da avaliação correta de consultas a bancos de dados com informação incompleta também tem sido objeto de estudo de muitos autores (p.ex. [LP76], [Lip79], [Rei86], [Sik81], [YC88]). Alguns deles, como [Rei86] utilizam-se da definição de bancos de dados relacionais através da lógica [LS88], [Rei78], [Rei84] para obter seus resultados.

Outros tipos de representação de informação incompleta, como "informações possíveis" são analisadas em [LS90, LS91]. Discute-se também o problema de valores vazios em bancos de dados não normalizados [RKS89] e em bancos de dados orientados a objetos [Zic90].

Neste texto apresentamos as diversas linhas de pesquisa na área de valores vazios em bancos de dados e comentamos os resultados obtidos. Existem ainda várias questões em aberto que certamente serão objeto de trabalho futuro. Entretanto, o texto se propõe a ser uma referência para se conhecer o assunto e problemas correlatos, na situação atual de pesquisas.

O texto está organizado da seguinte forma:

- No capítulo 2 apresentamos uma breve descrição do modelo relacional e de outros conceitos básicos que serão necessários para a leitura do restante do texto. Alguns desses conceitos serão objeto de estudo especial nos capítulos subsequentes.

- No capítulo 3 apresentamos os tipos de valores vazios mais utilizados e as lógicas multivaloradas desenvolvidas para lidar com os mesmos. 
- No capítulo 4 apresentamos as diversas extensões propostas para o modelo relacional para incorporar o tratamento de valores vazios. Neste capítulo apresentamos também algumas extensões da álgebra relacional, que fornecerão subsídios para o estudo do problema de avaliação de consultas a bancos de dados com valores vazios. Este talvez seja o assunto mais extensivamente estudado na área, devido à sua importância e complexidade. Basicamente, é preciso rever toda a álgebra relacional e as linguagens de acesso para se encontrar um resultado satisfatório.

- No capítulo 5 mostramos uma forma de representação do modelo relacional através da lógica de primeira ordem e como é feito o tratamento de valores vazios nessa representação.

- No capítulo 6 comentamos o problema de avaliação de consultas a bancos de dados com valores vazios e apresentamos algoritmos de consulta em representação lógica.

- No capítulo 7 apresentamos os problemas de atualização em bancos de dados com valores vazios.

- No capítulo 8 estudamos em detalhe um dos conceitos apresentados no capítulo 2 para o tratamento da negação em bancos de dados, para representar fatos do tipo "Juca não estuda medicina" ou "Juca não está matriculado em álgebra nem em geometria".

- No capítulo 9 descrevemos do tratamento de valores vazios em outros modelos de dados.

- No capítulo 10 apresentamos uma proposta de implementação de um SGBD com valores vazios.

- Finalmente, no capítulo 11 apresentamos algumas conclusões do texto, com os comentários finais.

A motivação para a realização desse texto foi uma palestra ministrada pelo professor Valdemar W. Setzer no Instituto de Matemática e Estatística da Universidade de São Paulo em 1993, denominada "O Modelo Relacional com Incerteza e sua Implementação Não-normalizada". A palestra não só motivou o preparo do texto como também foi fonte de valiosas referências bibliográficas. 


\section{Capítulo 2}

\section{Definições básicas}

O objetivo deste capítulo é apresentar os conceitos básicos utilizados ao longo do texto, além de uniformizar a notação adotada. Supomos que estes conceitos já são familiares ao leitor, de modo que esta apresentação será bastante sucinta, composta basicamente de definições e descrições de notação.

\subsection{Modelo Relacional}

O modelo relacional, como já foi mencionado, foi introduzido por Codd no início da década de 1970. Uma de suas grandes vantagens é representar os dados de maneira abstrata, menos influenciada pela forma de implementação. Isso o torna mais próximo do raciocínio lógico humano e menos da máquina. Os conceitos e exemplos apresentados aqui estão em [KS86], [Mai83].

Um esquema de relação $R$ é um conjunto finito de atributos $R=\left\{A_{1}, \ldots, A_{n}\right\}$. A cada atributo $A_{i}$ corresponde um conjunto $D_{i}$, que é o domínio, ou conjunto de valores possíveis para $A_{i}$, denotado por $\operatorname{Dom}\left(A_{i}\right)$. Uma relação $r$ num esquema $R$, denotado por $r(R)$, é um subconjunto finito $\left\{t_{1}, \ldots, t_{m}\right\}$ do produto cartesiano dos domínios, isto é, para $1 \leq j \leq m, t_{j} \in D_{1} \times D_{2} \times \ldots \times D_{n}$. Para denotar o valor do atributo $A$ ou do conjunto de atributos $X$ na tupla $t$, usamos $t[A]$ e $t[X]$.

Uma chave de uma relação $r(R)$ é um subconjunto $K=\left\{B_{1}, \ldots, B_{n}\right\}$ de $R$ tal que para duas tuplas quaisquer $t_{1}$ e $t_{2}$ distintas, $t_{1}[K] \neq t_{2}[K]$. Em outras palavras, isto significa que não existem duas tuplas diferentes que possuam o mesmo valor em todos os atributos em $K$. Além disso, o subconjunto $K$ é minimal, isto é, não existe $K^{\prime} \subseteq K$ com a mesma propriedade. 
Note que pode-se especificar mais de uma chave para a mesma relação. Nesse caso escolhemos uma das chaves para destacá-la como chave primária, o que produz uma organização interna do arquivo onde está armazenada a relação.

\section{2 Álgebra Relacional}

Existem 5 operações básicas na álgebra relacional: seleção, projeção, produto cartesiano, união e diferença. Todas elas produzem uma nova relação como resultado. Além dessas 5 básicas, existem ainda outras operações úteis para se formular consultas com mais facilidade e que serão definidas a partir das operações básicas : intersecção, tetajunção, junção natural e divisão. As operações de seleção e de projeção são unárias, isto é, atuam sobre uma única relação. As demais são binárias, ou seja, atuam sobre pares de relações.

A definição dos 5 operadores básicos é a seguinte:

- seleção: $\sigma_{\Theta}(r)=\{t \mid t \in r$ e $\Theta(t)\}$, onde $\Theta$ é uma expressão booleana envolvendo constantes e atributos no esquema de $r$.

- projeção: $\Pi_{X}(r)=\{t[X] \mid t \in r$ e $X \subseteq R\}$.

- produto cartesiano: $r_{1} \times r_{2}=\left\{t_{1} \& t_{2} \mid t_{1} \in r_{1}, t_{2} \in r_{2}\right\}$, onde "\&" representa a concatenação das duas tuplas.

- união: $r_{1} \cup r_{2}=\left\{t \mid t \in r_{1}\right.$ ou $\left.\mathrm{t} \in r_{2}\right\}$

- diferença: $r_{1}-r_{2}=\left\{t \mid t \in r_{1}\right.$ e t $\left.\notin r_{2}\right\}$

A partir dos operadores vistos, podemos definir formalmente uma expressão na álgebra relacional. Uma expressão básica é :

- uma relação no banco de dados, ou

- uma relação constante, isto é, um conjunto de tuplas em um esquema qualquer, que não será considerada como um elemento do banco de dados.

Como os operadores da álgebra relacional produzem sempre uma relação, uma expressão envolvendo estes operadores também produz uma relação. Dessa forma, podemos definir o esquema de uma expressão como o esquema da relação produzida pela expressão. 
A definição indutiva de expressões é feita da seguinte forma: sejam $E_{1}, E_{2}$ expressões da álgebra relacional. Então as seguintes construções também são expressões:

- $E_{1} \cup E_{2}$

- $E_{1}-E_{2}$

- $E_{1} \times E_{2}$

- $\sigma_{P}\left(E_{1}\right)$, onde $P$ é uma expressão booleana envolvendo atributos do esquema de $E_{1}$ e constantes.

- $\Pi_{S}\left(E_{1}\right)$, onde $S$ é um subconjunto de atributos do esquema de $E_{1}$.

Em alguns casos, ao se formular consultas, a expressão correspondente na álgebra relacional é muito comprida, tornando difícil sua compreensão. Para facilitar a manipulação dessas expressões, temos outros operadores relacionais que na verdade são "abreviações" de expressões mais complicadas. forma:

Os operadores adicionais são escritos em funcão dos operadores básicos da seguinte

- interseção: $r \cap s=r-(r-s)$

- teta-junção: $r \bowtie_{\Theta} s=\sigma_{\Theta}(r \times s)$

- junção natural: $r \bowtie s=\Pi_{R \cup S}\left(r \bowtie_{\Theta} s\right)$, onde $\Theta=\left(r\left[A_{1}\right]=s\left[A_{1}\right] \wedge \ldots \wedge r\left[A_{n}\right]=\right.$ $\left.s\left[A_{n}\right]\right)$, e $R \cap S=\left\{A_{1}, \ldots, A_{n}\right\}$

- divisão: $r \div s=\Pi_{R-S}(r)-\Pi_{R-S}\left(\left(\Pi_{R-S}(r) \times s\right)-r\right)$

\subsection{Dependências Funcionais e Restrições de In- tegridade}

Dizemos que a relação $r(R)$ satisfaz a dependência funcional $X \rightarrow Y$ (onde $X, Y \subseteq$ $R)$, se e somente se, para quaisquer tuplas $t_{1}$ e $t_{2}, t_{1}[X]=t_{2}[X]$ implica $t_{1}[Y]=t_{2}[Y]$

A relação $r(R)$ satisfaz a dependência multivalorada $X \rightarrow Y$ se e somente se, para duas tuplas distintas $t_{1}$ e $t_{2}$ em $r, t_{1}[\mathrm{X}]=t_{2}[X]$, implica que existe uma tupla $t_{3}$ em $r$ tal que : 
- $t_{3}[X]=t_{1}[X]$

- $t_{3}[Y]=t_{1}[Y]$

- $t_{3}[Z]=t_{2}[Z]$

onde $Z=R-(X \cup Y)$.

A simetria na igualdade de $t_{1}$ e $t_{2}$ implica que também deve existir uma tupla $t_{4}$ em $r$ tal que

- $t_{4}[X]=t_{2}[X]$

- $t_{4}[Y]=t_{2}[Y]$

- $t_{4}[Z]=t_{1}[Z]$

onde $Z=R-(X \cup Y)$.

As dependências multivaloradas são importantes porque, quando existe uma dependência $\mathrm{X} \rightarrow \mathrm{Y}$ numa relação $r(R)$, pode-se decompor $r$ sem perdas em duas relações de esquema $X Y$ e $Y Z$, onde $Z=R-(X \cup Y)$. Se $R=\{X, Y, Z\}$, uma decomposição de $r(R)$ sem perdas em $r_{1}(X Y)$ e $r_{2}(Y Z)$ é tal que $r=r_{1} \bowtie r_{2}$.

$\mathrm{O}$ último tipo de dependência a ser considerada é a dependência de junção. Seja $R=\left\{R_{1}, \ldots, R_{n}\right\}$ um conjunto de esquemas de relações tais que $R_{1} \cup \ldots \cup R_{n} \subseteq U$. Uma relação $r(U)$ satisfaz a dependência de junção (JD) $*\left[R_{1}, \ldots, R_{n}\right]$ se $r$ pode ser decomposta sem perdas em relações $\Pi_{R_{1}}(r), \ldots, \Pi_{R_{n}}(r)$, isto é, se $r=\Pi_{R_{1}}(r) \bowtie \ldots \bowtie$ $\Pi_{R_{n}}(r)$. A importância das dependências de junção pode ser explicada pelos mesmos motivos apresentados para as dependências multivaloradas. Por exemplo, a relação abaixo satisfaz a dependência de junção * $[A B, A C, B C]$ :

\begin{tabular}{|l|l|l|}
\hline $\mathrm{A}$ & $\mathrm{B}$ & $\mathrm{C}$ \\
\hline \hline$a_{1}$ & $b_{1}$ & $c_{1}$ \\
$a_{1}$ & $b_{2}$ & $c_{2}$ \\
$a_{3}$ & $b_{3}$ & $c_{3}$ \\
$a_{4}$ & $b_{3}$ & $c_{4}$ \\
$a_{5}$ & $b_{5}$ & $c_{5}$ \\
$a_{6}$ & $b_{6}$ & $c_{5}$ \\
\hline
\end{tabular}

Em geral, os conceitos de dependência (funcional, multivalorada ou de junção) permitem a identificação de redundância nas relações do banco de dados. A eliminação de redundâncias aumenta a confiabilidade dos dados, pois, se uma mesma 
informação ou dado aparece duplicado dentro de uma ou mais tabelas no banco, há a possibilidade de se alterar o valor do mesmo em uma tabela e não nas outras, levando a uma inconsistência nos dados.

A partir da definição de dependências, foram criadas as formas normais, cujo objetivo é eliminar redundâncias e outras anomalias no banco de dados. Para uma exposição completa das mesmas, veja [Mai83].

Para os propósitos desse texto, é suficiente conhecer o conceito de Primeira Forma Normal, que não é consequência das dependências, mas uma definição especial: Uma relação $r(R)$ está em Primeira Forma Normal quando todos os atributos $A \in R$ são atômicos. Um atributo não-atômico pode conter valores compostos ou um conjunto de valores compostos. Existe uma certa polêmica a respeito do uso dessa forma normal, mas está fora do escopo desse texto discuti-la.

Em [Codd70], Codd denominou as relações em Primeira Forma Normal simplesmente "relações normalizadas". Foi somente em [Codd72], ao introduzir os conceitos de Segunda e Terceira Formas Normais, que passou a utilizar essa terminologia. Uma relação com algum atributo não-atômico será denominada aqui de relação não normalizada (Primeira Forma Normal).

Além das dependências, existem outros tipo de restrições que podem aparecer em um banco de dados. Normalmente, não são restrições de uso geral como as formas normais. São usadas para exprimir restrições de ordem semântica no banco de dados e podem em geral ser escritas como sentenças na lógica de primeira ordem. Chamamos de restrições de integridade, ou simplesmente restrições, a estas sentenças lógicas que traduzem regras que o banco de dados deve satisfazer.

\subsection{O modelo relacional e a lógica}

Nos capítulos 5 e 8 apresentamos conceitos vistos nessa seção de forma mais completa. Como no entanto a terminologia definida aqui será utilizada em capítulos anteriores ao 5 , é necessário citar os conceitos e definições utilizados.

Observe o exemplo abaixo:

Funcionários
\begin{tabular}{|l|l|l|}
\hline Nome & Idade & Cargo \\
\hline \hline Fabio & 28 & analista \\
Marcos & 35 & gerente \\
Maria & 36 & secretária \\
\hline
\end{tabular}

\begin{tabular}{|l|l|}
\multicolumn{2}{c}{ Chefias } \\
\hline Chefe & Subordinado \\
\hline \hline Marcos & Fabio \\
Marcos & Maria \\
\hline
\end{tabular}


Poderíamos representar os mesmos fatos utilizando os predicados Funcionários e Chefias:

- Funcionários (Fabio, 28, analista)

- Funcionários (Marcos, 35, gerente)

- Funcionários (Maria, 36, secretária)

- Chefias (Marcos, Fabio)

- Chefias (Marcos, Maria)

Ou seja, é, verdade que (Fabio, 28, analista) pertence à relação Funcionários e assim por diante.

A idéia básica da representação de bancos de dados pela lógica é esta: a cada relação associa-se um predicado com tantas variáveis quantos forem os atributos da relação original. $\mathrm{O}$ banco de dados é o conjunto desses predicados, onde para cada tupla da relação temos o predicado correspondente com os dados da tupla.

\subsection{Hipóteses sobre Bancos de Dados: CWA e OWA}

Os conceitos de CWA (hipótese do mundo fechado, de Closed World Assumption) e de OWA (hipótese do mundo aberto, de Open World Assumption), introduzidos por Reiter [Rei78], são usados para definir o tratamento de fatos negativos. Suponha que se queira incluir no banco do exemplo os fatos:

- "Mario, analista de 28 anos, não é funcionário"

- "Fabio não é chefe de Maria"

Há duas formas de se tratar estes fatos:

- Não incluir nenhuma informação a seu respeito no banco de dados. Assume-se que todo fato que não está explicitamente representado é falso. Esta é a CWA. 
- Inclui-se no banco de dados tuplas "negativas", correspondentes aos predicados $\neg$ Funcionários (Mario, 25, analista) e $\neg$ Chefias (Fabio, Maria). Nenhum fato é assumido falso, a menos que sua negação esteja explicitamente representada. Esta é a OWA.

Nos bancos de dados convencionais, assume-se, em geral, a CWA. Porém, como veremos posteriormente, ao incorporar o tratamento de valores vazios, assumir a CWA traz alguns problemas. Isto será discutido no capítulo 8. 


\section{Capítulo 3}

\section{Valores vazios}

Em bancos de dados relacionais, a consequência de termos informações incompletas é a existência de atributos cujos valores não são especificados em algumas tuplas. Estes valores não especificados são denominados valores vazios.

É necessário definir como representar e como tratar os valores vazios. Sintaticamente, devem ser definidos símbolos especiais para os distinguir dos valores não vazios. Semanticamente, é importante detalhar o significado desses símbolos. Operacionalmente, deve ser definido como processar os mesmos.

\subsection{Tipos de valores vazios}

Apesar dos 13 tipos diferentes de valores vazios identificados na ANSI/X3/SPARC, na prática é necessário diferenciar apenas alguns; introduzimos a notação que os caracterizará neste trabalho:

- o valor vazio que representa um valor desconhecido mas aplicável $n_{A}$

- o valor vazio que representa um valor inaplicável $n_{I}$

- o valor vazio que representa um valor para o qual não há informação (não se sabe se é aplicável ou não) $n_{S}$

O conceito de "aplicabilidade" de um valor é fundamental para a distinção entre tais valores vazios. Basicamente, este conceito traduz a idéia de "existência" do valor. Um valor aplicável é um valor que existe, mas é desconhecido. Um valor inaplicável 
é um valor inexistente. Através do exemplo a seguir é mais fácil compreender estas idéias:

Funcionários

\begin{tabular}{|l|c|c|c|}
\hline Nome & Idade & Est.Civil & Nome do cônjuge \\
\hline \hline Silva, A.L. & 25 & solteiro & $n_{I}$ \\
Santos, J.F. & 30 & casado & $n_{A}$ \\
Marques, M. & 35 & $n_{A}$ & $n_{S}$ \\
\hline
\end{tabular}

Para a primeira tupla, o campo nome do cônjuge (legal) não existe, pois o funcionário é solteiro - este é o caso do valor vazio inaplicável. Para a segunda tupla, este valor existe, mas é desconhecido, pois o campo correspondente não foi preenchido este é o caso do valor vazio aplicável. Para a terceira tupla, como não se conhece o estado civil do funcionário, não se pode dizer se o valor é aplicável ou não - este é o caso do valor vazio sem informação.

As seções seguintes descrevem com mais detalhes estes tipos de valores vazios e apresentam os resultados dos trabalhos de pesquisa na área.

\subsubsection{O valor vazio aplicável}

O valor vazio aplicável é utilizado para se representar um valor que, apesar de ser desconhecido, sabe-se que existe e que portanto poderá futuramente ser alterado para um elemento qualquer do domínio do atributo.

Em seu primeiro trabalho para tratamento de valores vazios, Codd [Codd79] considerou apenas o valor vazio desconhecido mas aplicável, e usou a notação $\omega$. Maier [Mai83] também seguiu o mesmo caminho, mas usando a notação $\perp$. Ao longo deste texto, será utilizada a notação $n_{A}$.

Em 1986, Codd publicou um novo trabalho sobre o assunto [Codd86], detalhando mais o aspecto semântico dos valores vazios. Um ponto interessante deste trabalho é que ele descarta o uso de valores vazios para "deduzir" fatos no banco de dados. Segundo Codd, nos poucos casos onde isso é possível, o custo computacional de fazê-lo não justifica a quantidade de informação adquirida.

Entretanto, em [Mai83], Maier apresenta os valores vazios identificados como uma ferramenta para esse tipo de dedução. Os valores vazios identificados são tipos especiais do valor vazio $n_{A}$, onde é associado um número ou nome a cada ocorrência distinta do mesmo no banco de dados. Quando se sabe que dois valores vazios, apesar de não conhecidos, representam o mesmo elemento do domínio, associa-se a 
ambos um valor vazio com o mesmo identificador. A partir desse conhecimento, e de regras estruturais do banco (p.ex, dependências funcionais), pode-se deduzir alguma informação nova. Por exemplo, considere a seguinte relação:

\begin{tabular}{|l|l|l|l|l|}
\cline { 2 - 4 } \multicolumn{1}{c|}{} & $A$ & $B$ & $C$ & $D$ \\
\hline$t_{1}$ & $\mathrm{a}$ & $\mathrm{b}$ & $n_{A_{1}}$ & $n_{A_{2}}$ \\
$t_{2}$ & $\mathrm{a}$ & $n_{A_{3}}$ & $n_{A_{4}}$ & $\mathrm{~d}$ \\
\hline
\end{tabular}

Se considerarmos que esta relação deve satisfazer as dependências funcionais $\mathrm{A} \rightarrow \mathrm{C}, \mathrm{C} \rightarrow \mathrm{D}$, devemos igualar os valores vazios e constantes, de modo a satisfazer as dependências. Dessa forma, a relação fica:

\begin{tabular}{|l|l|l|l|l|}
\cline { 2 - 4 } \multicolumn{1}{c|}{} & $A$ & $B$ & $C$ & $D$ \\
\hline$t_{1}$ & $\mathrm{a}$ & $\mathrm{b}$ & $n_{A_{1}}$ & $\mathrm{~d}$ \\
$t_{2}$ & $\mathrm{a}$ & $n_{A_{3}}$ & $n_{A_{1}}$ & $\mathrm{~d}$ \\
\hline
\end{tabular}

Assim obtemos uma relação com menos valores vazios (e portanto mais informativa) que a relação original. Um argumento parecido com este é utilizados por Imielinski e Lipski [IL84] na definição das V-tabelas que serão apresentadas no capítulo 4.

\subsection{2 $\mathrm{O}$ valor vazio inaplicável}

O valor vazio inaplicável é utilizado para se representar um valor que sabidamente não existe. Este tipo de valor vazio não é alterado para nenhum elemento do domínio. Usaremos a notação $n_{I}$ para este valor vazio.

O valor vazio inaplicável $n_{I}$ recebe um tratamento diferenciado no trabalho de Lerat e Lipski [LL86]. Antes de se definir a forma de representação e o tratamento do mesmo, são colocadas diversas razões pelas quais tal tipo de valor vazio merece destaque. A principal delas é que, apesar de todos os tipos de valores vazios representarem certas anomalias dos dados, o valor vazio inaplicável é uma consequência da estrutura lógica dos dados. Argumenta-se que o uso de valores vazios inaplicáveis pode ser um reflexo de um mau projeto de banco de dados, o que não é verdade em todos os casos. São apresentadas 3 razões pelas quais deve-se aceitar o uso do valor vazio inaplicável:

1) O uso de valores vazios inaplicáveis é comum no dia a dia, como, por exemplo, no preenchimento de formulários. Por um lado, é quase impossível prever a estrutura lógica da informação antes de se projetar a estrutura lógica do banco de dados (no 
caso, o formulário). Mesmo que isso seja possível esta estrutura pode sofrer alterações ao longo do tempo. Por outro lado, às vezes é mais natural agrupar informações similares em um único formato, utilizando deliberadamente um "mau" projeto de banco de dados. Este agrupamento pode tornar o sistema mais agradável (por sua naturalidade) ao usuário final, e também reduzir redundâncias facilitando a manutenção da integridade dos dados.

2) O uso de valores vazios inaplicáveis pode ser um método para fornecer interfaces mais informativas com o usuário. Por exemplo, se temos um banco de dados com 2 relações, $r_{1}$ (Nome, Endereco) e $r_{2}$ (Nome, Nome de solteira) e uma consulta: Liste os nomes, endereços e nomes de solteira de todos os funcionários que não moram em São Paulo. Se em $r_{1}$ tivermos a tupla (Silva, Campinas) e não existir nenhum Silva na relação $r_{2}$, então na maioria dos casos o usuário gostaria de obter a resposta (Silva, Campinas, $n_{I}$ ) para não perder a informação contida em $r_{1}$.

3) No caso de se mudar o formato de uma relação, mais especificamente para se transformar uma relação não normalizada em uma relação normalizada (Primeira Forma Normal), a relação resultante deve poder conter vazios inaplicáveis, como no exemplo:

\begin{tabular}{|l|l|}
\hline Nome-Func & Nome-Depen \\
\hline \hline Silva & $\{$ Maria, Carlos $\}$ \\
Santos & $\Phi$ \\
\hline
\end{tabular}

$\Downarrow 1 \mathrm{a} . \mathrm{FN}$

\begin{tabular}{|l|l|}
\hline Nome-Func & Nome-Depen \\
\hline Silva & Maria \\
Silva & Carlos \\
Santos & $n_{I}$ \\
\hline
\end{tabular}

\subsubsection{O valor vazio desconhecido}

O valor vazio desconhecido é utilizado para se representar um valor que não se sabe se é aplicável ou não. $\mathrm{Na}$ verdade, os outros dois tipos de valores vazios são "desdobramentos" deste. Usamos a notação $n_{S}$ para este tipo de valor vazio.

Zaniolo [Zan84] propôs o emprego de apenas este tipo de valor vazio. Isso seria justificável porque : 
1. Este tipo de valor vazio representa exatamente o que acontece por exemplo na expansão de um esquema para incorporar mais um atributo. Se preenchermos este campo nas tuplas já existentes com o vazio aplicável ou com o vazio inaplicável, estaremos assumindo uma propriedade que ainda não se conhece (se é aplicável ou não). Nesse caso, o valor vazio $n_{S}$ é mais adequado.

2. O valor vazio sem informação $n_{S}$ incorpora os outros dois e a perda da informação sobre a aplicabilidade pode ser justificada pelo ganho em simplicidade de uso.

Por fim, Zaniolo conclui destacando que um banco de dados nunca representa a realidade com perfeição e que o nível de fidelidade é escolhido caso a caso. Assim, ao optar pelo uso do $n_{S}$, a aproximação com a realidade diminui, mas obtêm-se outros benefícios, principalmente simplicidade e eficiência.

Voltando ao trabalho de Codd [Codd86], destacam-se dois outros pontos de interesse: a classificação da falta de informação e o conceito de marcas para representar um valor vazio.

Quanto à classificação da falta de informação, Codd destaca duas questões: 1) A informação não representada é uma tupla inteira de uma relação ou apenas um subconjunto próprio de atributos em uma tupla? e 2) A informação não está representada porque não é conhecida ou porque é inaplicável? Estas questões são resumidas no quadro:

\begin{tabular}{|c|c|c|c|c|c|}
\hline \multicolumn{6}{|c|}{ Informação não representada } \\
\hline \multicolumn{3}{|c|}{ Tupla Completa } & \multicolumn{3}{c|}{ Atributos } \\
\hline $\begin{array}{c}\text { eventos } \\
\text { que não } \\
\text { ocorreram }\end{array}$ & desconhecidos & $\begin{array}{c}\text { eventos } \\
\text { não } \\
\text { aplicáveis }\end{array}$ & aplicáveis & não & outros \\
aplicáveis & \\
\hline
\end{tabular}

A idéia de usar marcas para representar valores vazios vem do fato que o significado de um valor vazio para um atributo não é o mesmo que o de um valor qualquer do domínio. Dessa forma, representar valores vazios usando valores do domínio não é adequado; as marcas substituem estes valores.

A utilização dos três tipos de valores vazios $n_{A}, n_{I}$ e $n_{S}$ simultaneamente é descrita em Roth et. al.[RKS89]. Segundo eles, esta é a aproximação mais completa e conceitualmente correta entre as que se conhece atualmente. A relação entre os valores vazios, em termos de quantidade de informação, pode ser resumida na figura a seguir: 


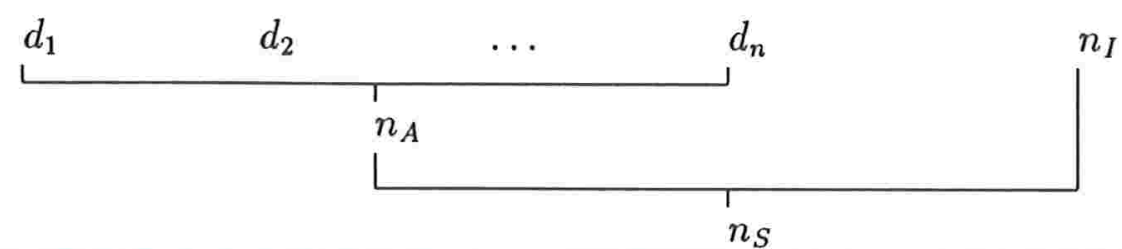

Isto é, o valor vazio $n_{S}$ é o que tem menos informação, $n_{A}$ e $n_{I}$ têm mais informação que $n_{S}$ e valores reais do domínio $d_{1}, \ldots, d_{n}$ têm mais informações que $n_{A}$.

Para calcular o valor de expressões envolvendo os valores vazios $n_{A}, n_{I}$ e $n_{S}$ e possibilitar assim consultas envolvendo essas expressões, muitos autores desenvolveram ferramentas lógicas diferentes. As próximas seções apresentam as principais delas.

\subsection{Lógica de 3 valores}

Codd [Codd79], ao estender o modelo relacional para incorporar o tratamento de valores vazios, considerou apenas o valor vazio aplicável $n_{A}$, que chamou de $\omega$. $\mathrm{Na}$ verdade, este era um tratamento preliminar que, segundo ele próprio, necessitava de pesquisas futuras.

Uma das primeiras questões analisadas foi a comparação de valores vazios. Por exemplo, qual o significado da expressão $x=y$ se $x$ ou $y$ for um valor vazio? Não se pode afirmar que a expressão é falsa ou verdadeira. Codd introduziu o valor lógico "pode ser", usando a mesma notação $\omega$. É necessário então uma lógica de 3 valores: Verdadeiro(V), Falso(F), ou Possível(P). No exemplo, a expressão $x=y$ assume o valor lógico $\mathbf{P}$. Os conectivos lógicos são definidos através das seguintes tabelas:

Conectivo e
\begin{tabular}{|c|c|c|c|}
\hline $\mathbf{e}$ & $\mathbf{F}$ & $\mathbf{P}$ & $\mathbf{V}$ \\
\hline $\mathbf{F}$ & $\mathbf{F}$ & $\mathbf{F}$ & $\mathbf{F}$ \\
$\mathbf{P}$ & $\mathbf{F}$ & $\mathbf{P}$ & $\mathbf{P}$ \\
$\mathbf{V}$ & $\mathbf{F}$ & $\mathbf{P}$ & $\mathbf{V}$ \\
\hline
\end{tabular}

Conectivo ou
\begin{tabular}{|c|c|c|c|}
\hline ou & F & P & V \\
\hline F & F & P & V \\
P & P & P & V \\
V & V & V & V \\
\hline
\end{tabular}

Conectivo não

\begin{tabular}{|c|c|}
\hline & não \\
\hline F & V \\
P & P \\
V & F \\
\hline
\end{tabular}


O valor lógico de uma expressão envolvendo o quantificador existencial é calculado como se a expressão fosse uma sequência de "ou"s, uma vez que o domínio de cada atributo é sempre um conjunto finito. Da mesma forma, o valor de uma expressão envolvendo o quantificador universal é calculado como se a expressão fosse uma sequência de "e".

As expressões envolvendo pertinência e inclusão em conjuntos são calculadas da seguinte forma: o valor de $n_{A} \in \mathrm{S}$ e de $\left\{n_{A}\right\} \subseteq \mathrm{S}$ é $\mathbf{P}$, mesmo que o valor vazio $n_{A}$ esteja no conjunto $\mathrm{S}$. Intuitivamente, atribui-se "Possível" a essas expressões porque não se conhece o valor do vazio $n_{A}$. Em outras palavras, $n_{A}$ pode ser um elemento qualquer do domínio, logo não se pode afirmar com certeza se $n_{A} \in \mathrm{S}$ ou $\left\{n_{A}\right\} \subseteq \mathrm{S}$.

Para definir a avaliação de uma expressão em que os termos contêm valores vazios, Codd introduziu o chamado "princípio da substituição de vazios", que atribui o valor lógico $\mathbf{P}$ se as seguintes condições são verdadeiras:

- Cada ocorrência do valor $n_{A}$ na expressão pode ser substituída por um valor não vazio (possivelmente um valor distinto para cada ocorrência), de forma que a expressão resultante seja Verdadeira (V).

- Cada ocorrência do valor $n_{A}$ na expressão pode ser substituída por um valor não vazio (possivelmente um valor distinto para cada ocorrência), de forma que a expressão resultante seja Falsa $(\mathbf{F})$.

Intuitivamente, o valor vazio $n_{A}$ é considerado como um "espaço a ser preenchido" por um elemento do domínio. Assim, se $n_{A}$ pode ser preenchido de forma a se obter uma expressão falsa e uma verdadeira, então nada pode ser afirmado a respeito da expressão, e por este motivo é atribuído a ela o valor Possível $(\mathbf{P})$.

O exemplo abaixo ilustra a utilização do princípio da substituição dos vazios para avaliação de expressões:
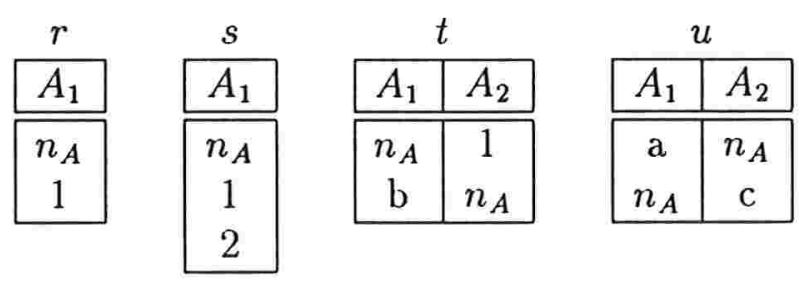

\begin{tabular}{|c|c|}
\multicolumn{2}{c}{$v$} \\
\hline$A_{1}$ & $A_{2}$ \\
\hline \hline $\mathrm{a}$ & $n_{A}$ \\
$\mathrm{~b}$ & 3 \\
$\mathrm{c}$ & 1 \\
\hline
\end{tabular}

- Expressões falsas: $n_{A} \in \Phi, t \subseteq s, v \subseteq u, u \subseteq r$.

- Expressões cujo valor lógico é P: $r \subseteq s, s \subseteq r, t \subseteq u, u \subseteq t, t \subseteq v, u \subseteq v$. 
Expressões envolvendo desigualdades $(\leq, \geq)$ entre dois elementos, onde pelo menos um deles é o valor vazio também resultam no valor lógico $\mathbf{P}$. Dessa forma, em uma ordenação o valor vazio $n_{A}$ não tem lugar pré-fixado.

Grant [Gra77] sugere o uso de uma lógica de 3 valores que não é lógico-funcional mas avalia corretamente tautologias da forma "Idade $\geq 50$ ou Idade $<50$ ", que segundo o autor devem ser avaliadas como $\mathbf{V}$ mesmo quando o valor de Idade é $n_{A}$. Nesse caso o valor da expressão não é determinado apenas pelo conectivo e pelos valores dos atributos. A sugestão é substituir cada ocorrência de um valor vazio por um valor do domínio: se o valor da expressão for $\mathbf{V}$ para todas as substituições possíveis, então atribui-se o valor $\mathbf{V}$ para a expressão; se o valor da expressão for $\mathbf{F}$ para todas as substituições, então atribui-se $\mathbf{F}$; nos outros casos atribui-se o valor $\mathbf{P}$.

\subsection{Lógica de 4 valores}

Na lógica de 3 valores é considerado apenas um tipo de valor vazio, o desconhecido mas aplicável $n_{A}$. Se quisermos incorporar além deste o valor vazio inaplicável $n_{I}$, devemos considerar uma lógica que faça a distinção entre estes valores. Por esse motivo, Gessert [Ges90] introduziu uma lógica de 4 valores : Inaplicável (I), aplicável e verdadeiro, que vamos chamar apenas de Verdadeiro (V), Possível (P), aplicável e falso, que chamaremos apenas de Falso (F).

A definição das tabelas-verdade para a lógica de 4 valores deve observar os diferentes tipos de negação que se pode definir O objetivo é definir essas tabelas coerentemente com a semântica desejada para os valores vazios. A interpretação utilizada é esquematizada da seguinte forma:

\begin{tabular}{|l|l|c|}
\hline Valor-verdade & \multicolumn{2}{|c|}{ Interpretação } \\
\hline Inaplicável (I) & & 0 \\
\hline Aplicável & Falso (F) & 1 \\
\cline { 2 - 3 } & Possível (P) & 2 \\
\cline { 2 - 3 } & Verdadeiro (V) & 3 \\
\hline
\end{tabular}

Segundo esse esquema, "mais verdadeiro" significa ter interpretação numericamente maior.

Existem vários tipos de negação em lógica multivalorada, porque "o inverso" de $\mathrm{V}$ não é simplesmente $\mathrm{F}$. Na lógica de 2 valores, por exemplo, se um fato não é verdadeiro, então é falso. Já na lógica de 4 valores, se um fato não é $\mathrm{V}$, não podemos precisar o seu valor-verdade, pois pode ser qualquer um dos outros três. 
Dentre as diversas possibilidades para definição de negação, temos por exemplo as tabelas inv e não:

\begin{tabular}{|l|c|}
\hline S & inv(S) \\
\hline $\mathbf{I}$ & V \\
F & P \\
$\mathbf{P}$ & $\mathbf{F}$ \\
V & I \\
\hline
\end{tabular}

\begin{tabular}{|c|c|}
\hline S & não(S) \\
\hline $\mathbf{I}$ & $\mathbf{I}$ \\
$\mathbf{F}$ & $\mathbf{V}$ \\
$\mathbf{P}$ & $\mathbf{P}$ \\
$\mathbf{V}$ & $\mathbf{F}$ \\
\hline
\end{tabular}

O primeiro conectivo (inv) corresponde a inverter completamente os valores lógicos $0,1,2,3$ e o segundo (não) corresponde a inverter somente os valores lógicos aplicáveis.

As tabelas-verdade dos conectivos e e ou são definidas com base nos seguintes conceitos: o conectivo e é o mínimo numérico dos valores-verdade, e o conectivo ou é o máximo. Para o cálculo de mínimo e máximo usamos os valores $(0,1,2,3)$ correspondendo, respectivamente a $(\mathbf{I}, \mathbf{F}, \mathbf{P}, \mathbf{V})$. Nesse caso, o valor inaplicável é considerado o "menos verdadeiro".

\begin{tabular}{|l|llll|}
\hline $\mathbf{e}$ & $\mathbf{I}$ & $\mathbf{F}$ & $\mathbf{P}$ & $\mathbf{V}$ \\
\hline $\mathbf{I}$ & $\mathbf{I}$ & $\mathbf{I}$ & $\mathbf{I}$ & $\mathbf{I}$ \\
$\mathbf{F}$ & $\mathbf{I}$ & $\mathbf{F}$ & $\mathbf{F}$ & $\mathbf{F}$ \\
$\mathbf{P}$ & $\mathbf{I}$ & $\mathbf{F}$ & $\mathbf{P}$ & $\mathbf{P}$ \\
$\mathbf{V}$ & $\mathbf{I}$ & $\mathbf{F}$ & $\mathbf{P}$ & $\mathbf{V}$ \\
\hline
\end{tabular}

\begin{tabular}{|l|llll|}
\hline ou & I & F & P & V \\
\hline I & I & F & P & V \\
F & F & F & P & V \\
P & P & P & P & V \\
V & V & V & V & V \\
\hline
\end{tabular}

ou, de maneira equivalente:

\begin{tabular}{|l|llll|}
\hline $\mathrm{e}$ & 0 & 1 & 2 & 3 \\
\hline 0 & 0 & 0 & 0 & 0 \\
1 & 0 & 1 & 1 & 1 \\
2 & 0 & 1 & 2 & 2 \\
3 & 0 & 1 & 2 & 3 \\
\hline
\end{tabular}

\begin{tabular}{|l|llll|}
\hline ou & 0 & 1 & 2 & 3 \\
\hline 0 & 0 & 1 & 2 & 3 \\
1 & 1 & 1 & 2 & 3 \\
2 & 2 & 2 & 2 & 3 \\
3 & 3 & 3 & 3 & 3 \\
\hline
\end{tabular}

Portanto, nessa lógica, se $|\mathrm{p}|$ representa o valor-verdade $(0,1,2,3)$ associado à sentença p, temos:

- $\mid p$ e $q \mid=\min (|p|,|q|)$

- $\mid p$ ou $q \mid=\max (|p|,|q|)$

- $|\operatorname{inv}(\mathrm{p})|=3-|\mathrm{p}|$ 
Note que, se considerarmos a lógica sem o valor inaplicável I, as tabelas correspondentes aos operadores e, ou e não são equivalentes às da lógica de 3 valores.

A interdefinibilidade de e eu

$$
\text { p ou } q=\text { não }(\text { não(p) e não(q)) }
$$

é válida na lógica de 4 valores somente se considerarmos inv como a negação. Temos então ( $\mathrm{p}$ ou $\mathrm{q}=\operatorname{inv}(\operatorname{inv}(\mathrm{p})$ e $\operatorname{inv}(\mathrm{q}))$ ). Essa regra não se aplica se for utilizado o não como negação.

Para ilustrar como são feitas consultas a um banco de dados utilizando expressões em lógica de 4 valores, Gessert [Ges90] apresenta alguns exemplos de consultas em linguagem SQL "estendida":

$$
\begin{aligned}
& \text { select Nome } \\
& \text { from Funcionários } \\
& \text { where } \mathrm{P}(\text { Salário } \geq 10000)
\end{aligned}
$$

Esta consulta lista os nomes de funcionários onde o campo Salário é aplicável, mas desconhecido.

$$
\begin{aligned}
& \text { select Nome } \\
& \text { from Funcionários } \\
& \text { where I(Salário = Salário) }
\end{aligned}
$$

Esta consulta lista os nomes de funcionários onde o campo Salário é inaplicável.

A consulta em SQL "normal"

select Nome

from Funcionários

where $($ Salário $=10000)$

é equivalente à consulta

select Nome

from Funcionários

where V(Salário = 10000) 
Dessa forma, as consultas em SQL "normal" são traduzidas para o SQL "estendido" utilizando o valor lógico $\mathrm{V}$.

Deve ser feito um tratamento especial para a negação, já que temos duas negações alternativas, não e inv. De modo geral, o conectivo não é aplicado quando, na sintaxe da linguagem, a palavra "not" aparece logo após a cláusula "where" ou nos casos especiais de consultas envolvendo apenas a negação e o valor lógico $\mathbf{V}$ (por exemplo, em consultas SQL "normais"). Os conectivos "and" e "or" na sintaxe do SQL são interpretados normalmente através dos operadores e e ou da lógica de 4 valores. Por exemplo, as consultas

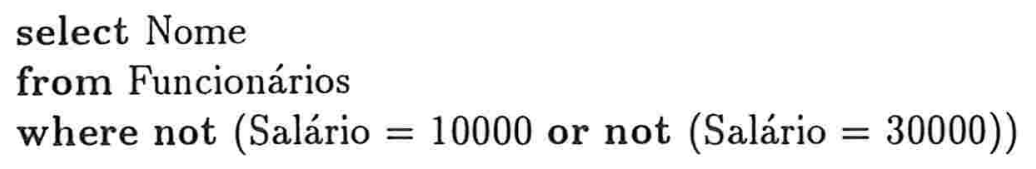

$\mathrm{e}$

select Nome

from Funcionários

where (Salário $\geq 10000$ ) and not (Salário $\geq 30000$ )

são interpretadas da forma:

select Nome

from Funcionários

where não (Salário $=10000$ ou inv $($ Salário $=30000)$

$\mathrm{e}$

select Nome

from Funcionários

where V (Salário $\geq 10000$ ) e não V(Salário $\geq 30000$ )

\subsection{Lógica de 7 valores e simplificações}

Para incorporar também o valor vazio "sem informação", $n_{S}$, é necessário tornar mais complexa a lógica. Para tanto, Yue [Yue91] definiu uma lógica multivalorada com os seguintes valores lógicos: 
- Verdadeiro (V)

- Falso (F)

- Inaplicável (I)

- Possível (P) - pode ser V ou F

- Sem Informação (S) - pode ser V ou F ou I

Aparentemente estes valores são suficientes para os 3 tipos de valores vazios. Entretanto, a situação é mais complicada, como se pode ver através do seguinte exemplo:

Considere uma relação Funcionários(Nome, Depto, Sal, Niv) onde Depto pode conter os 3 tipos de valores vazios, e a expressão

$$
\begin{gathered}
(\text { Depto }=\text { Contabilidade e } S a l \geq 20000) \text { ou } \\
(\text { Depto } \neq \text { Contabilidade }) \text { e }(N i v=7)
\end{gathered}
$$

Na tupla (Maria, $n_{S}, 30000,7$ ) o resultado da expressão pode ser $\mathbf{I}$ ou $\mathbf{V}$, mas nunca F. Se o campo Depto for inaplicável, o valor deve ser I. Por outro lado, se Depto for aplicável, então uma das sub-expressões (Depto $=$ Contabilidade) ou (Depto $\neq$ Contabilidade) será verdadeira. Como as sub-expressões $(\mathrm{Sal} \geq 20000)$ e $(\mathrm{Niv}=7)$ são ambas verdadeiras para a tupla, a expressão completa será verdadeira. Portanto, devemos ter mais um valor lógico, representando "Verdadeiro ou Inaplicável".

Da mesma forma, o valor da mesma expressão na tupla (Regina, $\left.n_{S}, 10000,4\right)$ pode ser $\mathbf{I}$ ou $\mathbf{F}$, mas nunca $\mathbf{V}$, pois o valor das sub-expressões $(S a l \geq 100000)$ e $(N i v=7)$ é F. É necessário, portanto, mais um valor lógico, "Falso ou Inaplicável".

Assim, para o tratamento dos 3 tipos de valores vazios $n_{A}, n_{I}$ e $n_{S}$ são necessários os 5 valores lógicos citados anteriormente mais os valores:

- Inaplicável ou Falso (IF)

- Inaplicável ou Verdadeiro (IV)

Pode-se entender a necessidade de 7 valores lógicos se considerarmos os valores básicos I, V e $\mathbf{F}$, e construirmos os valores lógicos a partir de todos os subconjuntos não vazios dos valores básicos: $\{\mathbf{I}\},\{\mathbf{F}\},\{\mathbf{V}\},\{\mathbf{I}, \mathbf{F}\},\{\mathbf{I}, \mathbf{V}\},\{\mathbf{F}, \mathbf{V}\}$ e $\{\mathbf{I}, \mathbf{F}, \mathbf{V}\}$. 
Uma lógica de 7 valores é difícil de ser utilizada. Por exemplo, a tabela-verdade dos conectivos e e ou tem 49 linhas. Além disso, é difícil (quase impossível) definir uma interpretação intuitiva dos conectivos nessa lógica. Por exemplo, qual o significado intuitivo de (IF ou IV)?

A simplificação da lógica de 7 valores é importante, pois como foi visto, é difícil de se trabalhar com a mesma. Uma simplificação óbvia, ainda segundo [Yue91], é usar apenas 2 valores básicos, V e F. Dessa forma obtém-se uma lógica de 3 valores $\{\mathbf{V}\}$, $\{\mathbf{F}\}$ e $\{\mathbf{V}, \mathbf{F}\}=\mathbf{P}$. Para obter apenas 2 valores básicos, iguala-se o valor $\mathbf{I}$ ao valor F. Assim, $\mathbf{V}$ representa o valor lógico Verdadeiro, e $\mathbf{F}$ representa os valores Falso e Inaplicável. Os outros valores lógicos são reduzidos da seguinte forma:

- $\mathbf{P}=\{\mathbf{F}, \mathbf{V}\}$

- $\mathbf{S}=\{\mathbf{I}, \mathbf{F}, \mathbf{V}\}=\{\mathbf{F}, \mathbf{F}, \mathbf{V}\}=\{\mathbf{F}, \mathbf{V}\}=\mathbf{P}$

- $\mathbf{I V}=\{\mathbf{I}, \mathbf{V}\}=\{\mathbf{F}, \mathbf{V}\}=\mathbf{P}$

- $\mathbf{I F}=\{\mathbf{I}, \mathbf{F}\}=\{\mathbf{F}, \mathbf{F}\}=\{\mathbf{F}\}=\mathbf{F}$

O exemplo a seguir ilustra a simplificação proposta:

A expressão (Depto=Contabilidade) pode ser interpretada como a consulta "quem trabalha no departamento de contabilidade?". Se o campo Depto de um funcionário contém o valor vazio $n_{I}$, então este funcionário não trabalha em departamento nenhum, e portanto, é natural que o valor da expressão seja $\mathbf{F}$, e este funcionário não apareça no resultado da consulta.

Pode-se definir então a tabela do operador igualdade, como descrito a seguir. Note que há uma diferença em relação ao tratamento do valor vazio $n_{I}$, que Codd tratou da mesma forma que $n_{S}$ e, portanto, indica incerteza. No entanto, não há nenhuma incerteza em relação a $n_{I}$, pois sabemos que o valor é inaplicável.

\begin{tabular}{l|c} 
Expressão & Valor \\
\hline$n_{S}=n_{S}$ & $\mathbf{P}$ \\
$n_{S}=n_{I}$ & $\mathbf{P}$ \\
$n_{S}=n_{A}$ & $\mathbf{P}$ \\
$n_{S}=$ "constante" & $\mathbf{P}$ \\
$n_{I}=n_{I}$ & $\mathbf{V}$ \\
$n_{I}=n_{A}$ & $\mathbf{F}$ \\
$n_{I}=$ "constante" & $\mathbf{F}$ \\
$n_{A}=n_{A}$ & $\mathbf{P}$ \\
$n_{A}=$ "constante" & $\mathbf{P}$
\end{tabular}


Analogamente, define-se a tabela para os operadores de desigualdade. Nesse caso, porém, o tratamento para o valor vazio $n_{I}$ é um pouco diferente, pois não há sentido na expressão " $n_{I}>n_{I}$ ", uma vez que a marca $n_{I}$ não pode ser ordenada. A interpretação dessa expressão seria "inaplicável”. Seguindo a regra de se igualar o valor I ao valor $\mathbf{F}$, o resultado dessa expressão será $\mathbf{F}$.

\begin{tabular}{l|c} 
Expressão & Valor \\
\hline$n_{S}>n_{S}$ & $\mathrm{P}$ \\
$n_{S}>n_{I}$ & $\mathrm{~F}$ \\
$n_{S}>n_{A}$ & $\mathrm{P}$ \\
$n_{S}>$ "constante" & $\mathrm{P}$ \\
$n_{I}>n_{I}$ & $\mathrm{~F}$ \\
$n_{I}>n_{A}$ & $\mathrm{~F}$ \\
$n_{I}>$ "constante" & $\mathrm{F}$ \\
$n_{A}>n_{A}$ & $\mathbf{P}$ \\
$n_{A}>$ "constante" & $\mathbf{P}$
\end{tabular}

As tabelas-verdade para os conectivos e e ou também devem ser definidas:

\begin{tabular}{|l|lll|}
\hline $\mathbf{e}$ & $\mathbf{F}$ & $\mathbf{P}$ & $\mathbf{V}$ \\
\hline $\mathbf{F}$ & $\mathbf{F}$ & $\mathbf{F}$ & $\mathbf{F}$ \\
$\mathbf{P}$ & $\mathbf{F}$ & $\mathbf{P}$ & $\mathbf{P}$ \\
$\mathbf{V}$ & $\mathbf{F}$ & $\mathbf{P}$ & $\mathbf{V}$ \\
\hline
\end{tabular}

\begin{tabular}{|l|lll|}
\hline ou & F & P & V \\
\hline F & F & P & V \\
P & P & P & V \\
V & V & V & V \\
\hline
\end{tabular}

\begin{tabular}{|c|c|}
\hline & não \\
\hline $\mathbf{F}$ & $\mathrm{V}$ \\
$\mathrm{P}$ & $\mathrm{P}$ \\
$\mathrm{V}$ & $\mathrm{F}$ \\
\hline
\end{tabular}

Após a simplificação, a lógica de 3 valores resultante não dispõe de ferramentas para identificar se uma expressão é aplicável ou não, pois o valor lógico I foi substituído por F. Caso seja necessária a distinção entre expressões aplicáveis e inaplicáveis, é preciso fornecer meios para que este teste possa ser feito.

Uma forma de se fazer esse teste é através de funções lógicas:

- Não-vazio(X) : retorna $\mathbf{V}$ se o atributo X é um valor não-vazio, e $\mathbf{F}$ caso contrário.

- Possível(X) : retorna $\mathrm{V}$ se o valor do atributo $\mathrm{X}$ é $n_{A}$, e $\mathbf{F}$ caso contrário.

- Inaplicável $(\mathrm{X})$ : retorna $\mathrm{V}$ se todos os atributos em $\mathrm{X}$ são inaplicáveis, $\mathbf{F}$ se todos aplicáveis e $\mathbf{P}$ nos outros casos.

O cálculo da função lógica Inaplicável é um pouco mais complexo que os outros e merece ser detalhado: 
1. Se X é um atributo ou uma constante, então:

(a) se $\mathrm{X}$ é $n_{I}$, então Inaplicável $(\mathrm{X})=\mathrm{V}$

(b) se $\mathrm{X}$ é $n_{S}$, então Inaplicável $(\mathrm{X})=\mathrm{P}$

(c) se $\mathrm{X}$ é $n_{A}$, então Inaplicável $(\mathrm{X})=\mathbf{F}$

(d) se $X$ é um valor não vazio, então Inaplicável $(X)=F$

2. Se $X$ é uma expressão $Y$ op-c $Z$ onde $Y$ e $Z$ são atributos ou constantes e op-c é um operador de comparação, então Inaplicável $(X)=$ Inaplicável(Y) ou Inaplicável(Z)

3. Se X é uma expressão $Y$ op-l Z onde Y e Z são expressões lógicas e op-l é um operador lógico, então Inaplicável $(X)=\operatorname{Inaplicável(Y)~ou~Inaplicável(Z)~}$

A partir dessas funções lógicas, podem-se definir outras para facilitar consultas, como Aplicável(X), Não-Verdadeiro(X), Não-Falso(X). Além disso, podem-se construir expressões que traduzem o significado dos 7 valores lógicos iniciais. Por exemplo, para calcular o valor $\mathbf{P}$ original, pode-se usar a expressão $X=(\operatorname{Possível}(X)$ e não Inaplicável(X))). A tabela abaixo mostra como calcular cada um dos 7 valores:

\begin{tabular}{l|l} 
Valor & Expressão \\
\hline V & X \\
F & $($ não X) e (não Inaplicável $(X))$ \\
I & Inaplicável(X) \\
P & (Possível(X) e (não Inaplicável(X))) \\
S & (Possível(X) e Possível (Inaplicável(X)) \\
IV & X ou Inaplicável(X) \\
IF & não X
\end{tabular}

Note que as funções lógicas Aplicável e Inaplicável também não são lógico-funcionais. Por exemplo, o valor da expressão (Aplicável(X) e Inaplicável $(X)$ ) é $\mathbf{P}$, se X contém o valor vazio $n_{S}$. No entanto, o valor dessa expressão obviamente deveria ser $\mathbf{F}$.

O problema de representação dos diferentes tipos de valores vazios, como se pode ver, é bastante complexo. Existem muitas dificuldades para o tratamento correto de expressões envolvendo valores vazios. A complexidade das expressões cresce de acordo com o número de tipos de valores vazios distintos utilizado. Por outro lado, o poder de representação dos fatos também é maior quando se utiliza mais tipos de valores vazios. A melhor solução deve ser definida de acordo com o tipo de aplicação. 


\section{Capítulo 4}

\section{Representações algébricas}

O significado e o comportamento dos valores vazios em expressões de comparação já foram descritos no capítulo anterior. Entretanto, ainda não foi analisada a forma de incorporar estes valores vazios no modelo relacional e quais os problemas decorrentes da utilização desses valores. Neste capítulo apresentamos as principais extensões propostas para o modelo relacional a fim de incorporar o uso dos valores vazios. Os modelos aqui apresentados são descritos utilizando um formalismo algébrico e uma notação tabular para relações. No próximo capítulo veremos como este problema pode ser analisado usando um formalismo lógico.

Ainda neste capítulo apresentamos modelos de dados que permitem a representação de informação disjuntiva, como por exemplo, fatos do tipo "Mario é médico ou enfermeiro". Esses modelos de dados também utilizam o formalismo algébrico para sua descrição.

Ao longo desse capítulo utilizaremos a seguinte notação:

- $r, s$ para relações

- $A, B, C$ para atributos; $X, Y, Z$ para conjuntos de atributos

- $t$ para tuplas

\subsection{Relações com valores vazios}

O modo mais simples de se estender o modelo relacional é permitir que cada atributo de uma relação possa assumir, além de valores no domínio do atributo, um dentre 
os valores vazios $n_{A}, n_{I}$ e $n_{S}$. Como já foi visto no capítulo anterior, quanto maior a variedade de tipos de valores vazios maior a complexidade para tratar expressões de comparação entre os mesmos. No caso de uso dos 3 tipos de valores vazios, sem nenhuma simplificação, utilizamos uma lógica de 7 valores.

Um outro problema a ser considerado é a redefinição dos operadores da álgebra relacional. Uma vez que o resultado da interpretação de expressões de comparação pode ser agora valores-verdade do tipo $\mathbf{V}, \mathbf{F}, \mathbf{P}, \mathbf{I}, \mathbf{S}, \mathbf{I V}, \mathbf{I F}$, a interpretação de expressões contendo operadores relacionais deve sofrer alterações.

Um dos primeiros trabalhos nesse sentido foi feito por Codd [Codd79, Codd86], que ao incorporar o valor vazio $n_{S}$ em suas relações definiu a lógica de 3 valores para expressões de comparação e também as alterações necessárias nos operadores relacionais.

Os operadores de união, intersecção e diferença não sofreram muita alteração. $O$ único ponto extra a ser considerado é que as tuplas são consideradas iguais se contêm os mesmos valores (vazios ou não vazios) nos atributos correspondentes. Isso pode parecer contraditório, se lembrarmos que na lógica de 3 valores o resultado da expressão $n_{A}=n_{A}$ é Possível, e não Verdadeiro. A justificativa é que a identificação de tuplas para remoção de duplicidade é uma necessidade básica do modelo relacional, pois, por definição, não podem existir duas tuplas idênticas em uma relação. Assim, duas tuplas idênticas, mesmo que possam ser semanticamente distintas, sintaticamente são iguais e portanto não se pode permitir a existência de ambas na mesma relação. Adotando essa regra, a definição desses operadores é a usual. Os exemplos abaixo ilustram seu comportamento (onde $r$ e $s$ são duas relações definidas no esquema $E(A, B))$ :

\begin{tabular}{|c|c|}
\multicolumn{2}{c}{$r$} \\
\hline$A$ & $B$ \\
\hline \hline$n_{A}$ & $n_{A}$ \\
$\mathrm{a}$ & $n_{A}$ \\
$\mathrm{a}$ & 1 \\
$n_{A}$ & 1 \\
\hline
\end{tabular}

\begin{tabular}{|c|c|}
\multicolumn{2}{c}{$s$} \\
\hline$A$ & $B$ \\
\hline \hline$n_{A}$ & $n_{A}$ \\
$\mathrm{a}$ & $n_{A}$ \\
$\mathrm{a}$ & 1 \\
\hline
\end{tabular}

\begin{tabular}{|c|c|}
\multicolumn{2}{c}{$r \cup s$} \\
\hline$A$ & $B$ \\
\hline \hline$n_{A}$ & $n_{A}$ \\
$\mathrm{a}$ & $n_{A}$ \\
$\mathrm{a}$ & 1 \\
$n_{A}$ & 1 \\
\hline
\end{tabular}

\begin{tabular}{|c|c|}
\multicolumn{1}{c}{$r \cap s$} \\
\hline$A$ & $B$ \\
\hline \hline$n_{A}$ & $n_{A}$ \\
$\mathrm{a}$ & $n_{A}$ \\
$\mathrm{a}$ & 1 \\
\hline
\end{tabular}

\begin{tabular}{|c|c|}
\multicolumn{2}{c}{$r-s$} \\
\hline$A$ & $B$ \\
\hline \hline$n_{A}$ & 1 \\
\hline
\end{tabular}

A definição do operador produto cartesiano não é afetada. O operador de projeção funciona da forma usual, mas a remoção de tuplas duplicadas segue a mesma regra usada para os operadores de união, intersecção e diferença. O exemplo seguinte ilustra a projeção: 


\begin{tabular}{|c|c|c|c|c|c|}
\hline \multicolumn{3}{|c|}{$r$} & \multicolumn{2}{|c|}{$\Pi_{B, C}(r)$} & $\Pi_{C}(r)$ \\
\hline$A$ & $B$ & $C$ & $B$ & $C$ & $C$ \\
\hline a & $n_{A}$ & $n_{A}$ & $n_{A}$ & $n_{A}$ & $n_{A}$ \\
\hline b & 1 & $n_{A}$ & 1 & $n_{A}$ & 1 \\
\hline$n_{A}$ & $n_{A}$ & 1 & $n_{A}$ & 1 & \\
\hline c & 1 & $n_{A}$ & & & \\
\hline d & $n_{A}$ & 1 & & & \\
\hline
\end{tabular}

$O$ operador teta-junção envolve uma expressão de comparação $\Theta$. O resultado dessa expressão pode ser agora $\mathbf{V}, \mathbf{F}$ ou $\mathbf{P}$. São criadas duas versões do operador: a teta-junção Verdadeira e a teta-junção Possível. A primeira retorna a relação onde a condição $\Theta$ é Verdadeira em todas as tuplas. A segunda retorna a relação onde a condição é Possível.

Assim como a teta-junção, a seleção também tem duas versões, a verdadeira e a possível. Vamos usar a notação $\mathbf{V}$ e $\mathbf{P}$ subscrita no operador para diferenciar as duas versões. Os exemplos abaixo ilustram o funcionamento desses operadores:

Relações iniciais:

\begin{tabular}{|c|c|}
\multicolumn{1}{c}{$r$} \\
\hline$A$ & $B$ \\
\hline \hline $\mathrm{a}$ & $n_{A}$ \\
$n_{A}$ & 2 \\
$n_{A}$ & 1 \\
\hline
\end{tabular}

\begin{tabular}{|c|}
$s$ \\
\hline$C$ \\
\hline$n_{A}$ \\
2 \\
\hline
\end{tabular}

Junção:

\begin{tabular}{|c|c|c|}
\multicolumn{1}{c}{$r_{{ }^{\star}} \mathrm{V}_{(B=C)}$} \\
\hline$A$ & $B$ & $C$ \\
\hline \hline$n_{A}$ & 2 & 2 \\
\hline
\end{tabular}

\begin{tabular}{|c|c|c|}
$r_{\bowtie} \mathrm{P}_{(B=C)}$ \\
\hline$A$ & $B$ & $C$ \\
\hline \hline $\mathrm{a}$ & $n_{A}$ & $n_{A}$ \\
$\mathrm{a}$ & $n_{A}$ & 2 \\
$n_{A}$ & 2 & $n_{A}$ \\
$n_{A}$ & 2 & 2 \\
$n_{A}$ & 1 & $n_{A}$ \\
\hline
\end{tabular}


Seleção:

\begin{tabular}{|c|c|}
$\sigma_{\mathrm{V}(B=2)}(r)$ \\
\hline$A$ & $B$ \\
\hline \hline$n_{A}$ & 2 \\
\hline
\end{tabular}

\begin{tabular}{|c|c|}
$\sigma_{\mathbf{P}(B=2)}(r)$ \\
\hline$A$ & $B$ \\
\hline \hline $\mathrm{a}$ & $n_{A}$ \\
$n_{A}$ & 2 \\
\hline
\end{tabular}

O operador de divisão é definido de forma idêntica. São criadas duas versões do operador, a Verdadeira, onde o teste de inclusão resulta $\mathrm{V}$, e a possível onde o teste resulta $\mathbf{P}$. O exemplo abaixo ilustra as duas versões:

Relações iniciais:

\begin{tabular}{|c|c|}
\multicolumn{1}{c}{$r$} \\
\hline$A$ & $B$ \\
\hline \hline $\mathrm{a}$ & 1 \\
$\mathrm{a}$ & 2 \\
$\mathrm{a}$ & 3 \\
$\mathrm{~b}$ & 2 \\
$\mathrm{~b}$ & $n_{A}$ \\
$\mathrm{c}$ & 3 \\
\hline
\end{tabular}
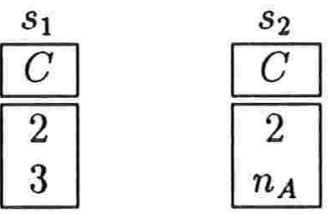

Divisão:

\begin{tabular}{|c|}
$r[\mathrm{~B} \div \mathrm{v} \mathrm{C}] s_{1}$ \\
\hline$A$ \\
\hline $\mathrm{a}$ \\
\hline
\end{tabular}

\begin{tabular}{|c|}
$r\left[\mathrm{~B} \div \mathrm{PC}_{\mathrm{P}} \mathrm{C}\right] s_{1}$ \\
\hline$A$ \\
\hline \hline $\mathrm{b}$ \\
\hline
\end{tabular}

\begin{tabular}{|c|}
$r[\mathrm{~B} \div \mathrm{V} C] s_{2}$ \\
\hline$A$ \\
\hline \hline$\Phi$ \\
\hline
\end{tabular}

\begin{tabular}{|c|}
$r\left[\mathrm{~B} \div \mathrm{PC}^{\mathrm{C}}\right] s_{2}$ \\
\hline$A$ \\
\hline $\mathrm{a}$ \\
$\mathrm{b}$ \\
\hline
\end{tabular}

Codd definiu ainda versões "externas" de alguns operadores. Nos operadores de união, interseç̧ão e diferença, a versão "externa" permite que esses operadores atuem sobre relações não totalmente compatíveis, compatibilizando-as através da inserção de valores vazios quando necessário. Mais formalmente, sejam dois esquemas $R(A, B)$ e $S(B, C)$ e $r(R), s(S)$ relações sobre os esquemas $R$ e $S$. Sejam também: 
- $r_{1}(A, B, C)=r \times\left(C: n_{A}\right)$

- $r_{2}(A, B, C)=\left(A: n_{A}\right) \times s$

onde $\times$ representa o produto cartesiano e $\left(C: n_{A}\right)$ indica uma relação $r(C)$ de uma única tupla onde todos os atributos em $C$ são o valor vazio $n_{A}$. A união externa de $r$ e $s$ é

$$
r \cup_{x} s=r_{1} \cup r_{2} \text {. }
$$

Analogamente definem-se interseç̧ão e diferença. O exemplo seguinte ilustra o operador de união externa:

\begin{tabular}{|c|c|c|}
\multicolumn{3}{c}{$r$} \\
\hline$A$ & $B$ & $C$ \\
\hline \hline $\mathrm{a}$ & 1 & 2 \\
$\mathrm{a}$ & 2 & 1 \\
$\mathrm{~b}$ & 1 & 2 \\
\hline
\end{tabular}

\begin{tabular}{|c|c|}
\multicolumn{1}{c}{$s$} \\
\hline$C$ & $D$ \\
\hline \hline 2 & $\mathrm{c}$ \\
3 & $\mathrm{~d}$ \\
\hline
\end{tabular}

\begin{tabular}{|c|c|c|c|}
\multicolumn{5}{c}{$r \cup_{x} s$} \\
\hline$A$ & $B$ & $C$ & $D$ \\
\hline \hline $\mathrm{a}$ & 1 & 2 & $n_{A}$ \\
$\mathrm{a}$ & 2 & 1 & $n_{A}$ \\
$\mathrm{~b}$ & 1 & 2 & $n_{A}$ \\
$n_{A}$ & 2 & $n_{A}$ & $\mathrm{c}$ \\
$n_{A}$ & 3 & $n_{A}$ & $\mathrm{~d}$ \\
\hline
\end{tabular}

Os operadores de junção externa utilizam valores vazios para que não haja perda de informação das tuplas das relações concatenadas. Formalmente, sejam $r\left(A, B_{1}\right)$ e $s\left(B_{2}, C\right)$ relações tais que $B_{1}$ e $B_{2}$ estejam definidos no mesmo domínio; $\Theta$ a expressão $\left(B_{1}=B_{2}\right)$. Sejam

- $r s=r \aleph_{\Theta} s$

- $r_{1}=r-\Pi_{A, B_{1}}(r s)$

- $s_{1}=s-\Pi_{B_{1}, C}(r s)$

A teta-junção externa de $r$ e $s$, representada por $\bowtie_{x_{\Theta}}$ é:

$$
r \bowtie_{x_{\Theta}} s=r s \cup\left(r_{1} \times\left(B_{2}: n_{A}, C: n_{A}\right)\right) \cup\left(\left(A: n_{A}, B_{1}: n_{A}\right) \times s_{1}\right)
$$

Exemplo: 


\begin{tabular}{|l|l|}
\multicolumn{2}{c}{$r$} \\
\hline$A$ & $C$ \\
\hline \hline$a_{1}$ & $c_{4}$ \\
$a_{2}$ & $c_{2}$ \\
$a_{4}$ & $c_{1}$ \\
$a_{6}$ & $c_{1}$ \\
$a_{7}$ & $c_{3}$ \\
\hline
\end{tabular}

\begin{tabular}{|l|l|}
\multicolumn{2}{c}{$s$} \\
\hline$C_{1}$ & $B$ \\
\hline \hline$c_{1}$ & $b_{1}$ \\
$c_{2}$ & $b_{2}$ \\
$c_{2}$ & $b_{2}$ \\
$c_{5}$ & $b_{4}$ \\
\hline
\end{tabular}

\begin{tabular}{|c|c|c|c|}
\multicolumn{5}{c}{$r \bowtie_{x_{C=C_{1}}} s$} \\
\hline$A$ & $C$ & $C_{1}$ & $B$ \\
\hline \hline$a_{1}$ & $c_{4}$ & $n_{A}$ & $n_{A}$ \\
$a_{2}$ & $c_{2}$ & $c_{2}$ & $b_{2}$ \\
$a_{2}$ & $c_{2}$ & $c_{2}$ & $b_{3}$ \\
$a_{4}$ & $c_{1}$ & $c_{1}$ & $b_{1}$ \\
$a_{6}$ & $c_{1}$ & $c_{1}$ & $b_{1}$ \\
$a_{7}$ & $c_{3}$ & $n_{A}$ & $n_{A}$ \\
$n_{A}$ & $n_{A}$ & $c_{5}$ & $b_{4}$ \\
\hline
\end{tabular}

A junção natural externa é definida analogamente, observando-se que, na remoção das colunas iguais, se houver dois campos, um vazio e outro não vazio, obviamente remove-se o valor vazio. No exemplo acima, se o esquema da relação $s$ fosse $(C, B)$ ao invés de $\left(C_{1}, B\right)$, a junção ficaria:

\begin{tabular}{|c|c|c|}
\multicolumn{4}{c}{$r \bowtie_{x} s$} \\
\hline$A$ & $C$ & $B$ \\
\hline \hline$a_{1}$ & $c_{4}$ & $n_{A}$ \\
$a_{2}$ & $c_{2}$ & $b_{2}$ \\
$a_{2}$ & $c_{2}$ & $b_{3}$ \\
$a_{4}$ & $c_{1}$ & $b_{1}$ \\
$a_{6}$ & $c_{1}$ & $b_{1}$ \\
$a_{7}$ & $c_{3}$ & $n_{A}$ \\
$n_{A}$ & $c_{5}$ & $b_{4}$ \\
\hline
\end{tabular}

Para os dois valores vazios $n_{A}$ e $n_{I}$ Codd [Codd86] propôs utilizar o mesmo tratamento, tanto na lógica de 3 valores quanto nos operadores relacionais.

O trabalho de Zaniolo introduz não só a noção de valor vazio sem informação $n_{S}$, mas também outros conceitos importantes para manipulação de relações com valores vazios. Um dos principais é a comparação da quantidade de informação em tuplas e relações: Uma tupla $t_{1}$ é mais informativa que $t_{2}$ (onde $t_{1} \in r(R), t_{2} \in s(S)$ ) se para todo atributo $A \in S$, se $t_{2}[A] \neq n_{S}$ então $A \in R$ e $t_{1}[A]=t_{2}[A]$. Notação: $t_{1} \geq t_{2}$

Dizemos que $t_{2}$ é menos informativa que $t_{1}$ se $t_{1} \geq t_{2}$. Se $t_{1} \geq t_{2}$ e $t_{2} \geq t_{1}$, dizemos que $t_{1}$ e $t_{2}$ são equivalentes. Notação: $t_{1} \cong t_{2}$.

É interessante notar que, na definição, $R \neq S$ permite a comparação de tuplas em esquemas diferentes, o que é muito útil no caso de esquemas onde se acrescentaram atributos. $\mathrm{O}$ exemplo abaixo ilustra as definições apresentadas: 


\begin{tabular}{|c|c|c|c|c|}
\multicolumn{5}{c}{ Funcionários1 } \\
\cline { 2 - 5 } \multicolumn{1}{c|}{} & No. & Nome & Sexo & Gerente \\
\hline$t_{1}$ & 1 & Silva & $n_{S}$ & 5 \\
$t_{2}$ & 1 & Silva & $\mathrm{F}$ & 5 \\
\hline
\end{tabular}

\begin{tabular}{|c|c|c|c|c|c|}
\multicolumn{7}{c}{ Funcionários2 } \\
\cline { 2 - 6 } \multicolumn{1}{c|}{} & No. & Nome & Sexo & Gerente & Fone \\
\hline$t_{3}$ & 1 & Silva & $\mathrm{F}$ & 5 & $n_{S}$ \\
$t_{4}$ & 1 & Silva & $\mathrm{F}$ & 7 & 654534 \\
\hline
\end{tabular}

Temos: $t_{2} \geq t_{1}, t_{2} \cong t_{3}, t_{3} \leq t_{4}$.

É adotada a seguinte convenção: se $t$ é uma tupla em $r(R)$, então $t[B]=n_{S}$, para todo atributo $B \notin R$. As tuplas que têm valores vazios em todos os seus atributos são chamadas de tuplas vazias. Uma tupla sem valores vazios é chamada total, e uma tupla $t \in r(R)$ sem valores vazios em $X \subseteq R$ é chamada $X$-total. Uma tupla $t$ é chamada encontro de duas tuplas $t_{1}$ e $t_{2}$, representado por $t \cong t_{1} \wedge t_{2}$, se para cada atributo:

- $t[A]=t_{1}[A]$ se $t_{1}[A]=t_{2}[A]$

- $t[A]=n_{S}$ se $t_{1}[A] \neq t_{2}[A]$

O encontro de duas tuplas é sempre mais informativo que qualquer tupla menos informativa que $t_{1}$ e $t_{2}$.

Dadas duas tuplas $t_{1}$ e $t_{2}$, é sempre possível obter a tupla correspondente ao encontro de ambas. A situação não é a mesma se considerarmos a junção (natural) de duas tuplas. Na verdade, a junção de duas tuplas é um caso particular do encontro destas tuplas. Duas tuplas $t_{1}$ e $t_{2}$ são passíveis de junção se, para cada atributo $A$, se $t_{1}[A] \neq t_{2}[A]$, então $t_{1}[A]=n_{S}$ ou $t_{2}[A]=n_{S}$. A junção de duas tuplas passíveis de junção $t_{1}$ e $t_{2}, \mathrm{t} \cong t_{1} \bowtie t_{2}$ é tal que:

- $t[A]=t_{1}[A]$, se $t_{1}[A] \geq t_{2}[A]$, ou

- $t[A]=t_{2}[A]$, se $t_{2}[A] \geq t_{1}[A]$

Desprezando as tuplas equivalentes, pode-se falar em junção ou encontro de duas tuplas. A junção de duas tuplas $t_{1}$ e $t_{2}$ é a tupla menos informativa entre as tuplas 
mais informativas que $t_{1}$ e $t_{2}$. O encontro de duas tuplas $t_{1}$ e $t_{2}$ é a tupla mais informativa entre as tuplas menos informativas que $t_{1}$ e $t_{2}$.

$\mathrm{O}$ conceito de tupla mais informativa pode ser estendido para relações. Uma relação $r_{1}$ é mais informativa que $r_{2}$ se, para toda tupla não vazia $t_{2} \in r_{2}$ existe uma tupla $t_{1} \in r_{1}$ tal que $t_{1} \geq t_{2}$. Notação: $r_{1} \succeq r_{2}$. Se $r_{1} \succeq r_{2}$ e $r_{2} \succeq r_{1}$, dizemos que $r_{1}$ e $r_{2}$ são equivalentes em quantidade de informação. Notação: $r_{1} \cong r_{2}$.

Intuitivamente, uma classe de equivalência de relações agrupa todas as relações que contêm a mesma quantidade de informação. Por exemplo, considere a relação $r(A, B, C)$ a seguir:

\begin{tabular}{|c|c|c|}
\hline$A$ & $B$ & $C$ \\
\hline \hline$a_{1}$ & $b_{1}$ & $c_{1}$ \\
$a_{2}$ & $b_{2}$ & $c_{2}$ \\
$a_{3}$ & $b_{3}$ & $n_{S}$ \\
\hline
\end{tabular}

Na mesma classe de equivalência de $r$ estão as relações:

\begin{tabular}{|c|c|c|c|}
\hline$A$ & $B$ & $C$ & $D$ \\
\hline \hline$a_{1}$ & $b_{1}$ & $c_{1}$ & $n_{S}$ \\
$a_{2}$ & $b_{2}$ & $c_{2}$ & $n_{S}$ \\
$a_{3}$ & $b_{3}$ & $n_{S}$ & $n_{S}$ \\
\hline
\end{tabular}

\begin{tabular}{|c|c|c|c|}
\hline$A$ & $B$ & $C$ & $E$ \\
\hline \hline$a_{1}$ & $b_{1}$ & $c_{1}$ & $n_{S}$ \\
$a_{2}$ & $b_{2}$ & $c_{2}$ & $n_{S}$ \\
$a_{3}$ & $b_{3}$ & $n_{S}$ & $n_{S}$ \\
\hline
\end{tabular}

A partir desses conceitos Zaniolo definiu as relações estendidas, que chamou de $\mathrm{x}$-relações. As x-relações são as classes de equivalência induzidas pelo operador $\cong$. Como usual quando se trabalha com classes de equivalência, toma-se um elemento da classe como seu representante. A classe de relações equivalentes a $r$, é denotada por $\hat{r}$ e $r$ é dito seu representante. Todos os conceitos da álgebra relacional são revistos para operar sobre x-relações. Esta é a extensão proposta para o modelo relacional com valores vazios. Veremos agora como foram revistos os operadores da álgebra relacional.

Uma tupla $t$ x-pertence a $\hat{r}$ (notação: $t \tilde{\epsilon} \hat{r}$ ) se, para algum $r^{\prime} \in \hat{r}, t \in r^{\prime}$.

Dado um conjunto de tuplas $\left\{t_{1}, \ldots, t_{n}\right\}$, pode-se remover tuplas menos informativas que outras e expandir tuplas com menor número de atributos colocando-se $n_{S}$ nas posições dos mesmos. O resultado é uma x-relação $\left\{t_{1}, \ldots, t_{n}\right\}$. Com essa notação, definimos então: 
- união: $\hat{r}_{1} \cup \hat{r}_{2}=\left\{t \mid t \in r_{1}\right.$ ou $t \in r_{2} \hat{\}}$

- intersecção: $\hat{r}_{1} \cap \hat{r}_{2}=\hat{\{} t \mid t \in r_{1}$ e $t \in r_{2} \hat{\}}$

- diferença: $\hat{r}_{1}-\hat{r}_{2}=\left\{t \mid t \in r_{1}\right.$ e $\left.t \notin r_{2}\right\}$

Essas definições são apropriadas no sentido em que preservam diversas propriedades que haviam sido perdidas na extensão proposta por Codd, tais como $\hat{r}_{1} \subseteq \hat{r}_{1} \cup \hat{r}_{2}$, $\hat{r}_{1} \cap \hat{r}_{2} \subseteq \hat{r}_{2}, \hat{r}_{1} \cong \hat{r}_{2}$.

A representação mínima de uma x-relação $\hat{r}$ é uma representação $r$ de $\hat{r}$ tal que não exista $r^{\prime} \subseteq r$ e $r^{\prime}$ seja também representação de $\hat{r}$. A representação mínima é única, e por este motivo é uma boa escolha para representante de $\hat{r}$.

O valor de expressões envolvendo valores vazios é calculado segundo o seguinte princípio: o valor vazio $n_{S}$ não satisfaz nenhuma expressão de comparação (isto é, envolvendo os operadores $=, \neq, \leq)$. Dessa forma evita-se o problema de detectar tautologias em consultas.

Os principais operadores relacionais são então definidos da seguinte forma:

- seleção: $\sigma_{\Theta}(r)=\hat{\{} t \mid t \in r$ e a expressão $\Theta$ é válida em $t$ e $t$ é total em todos os atributos envolvidos em $\Theta \hat{\}}$

- produto cartesiano: $\hat{r}_{1} \times \hat{r}_{2}=\hat{\{} t_{1} \vee t_{2} \mid t_{1} \in r_{1}$ e $t_{2} \in r_{2}$ e $t_{1}$ e $t_{2}$ não são vazios $\hat{\}}$ (onde o símbolo $\vee$ denota a concatenação de duas tuplas)

- projeção: $\Pi_{X}(r)=\hat{\{} t[X] \mid t \in r \hat{\}}$

- junção natural: $\hat{r}_{1} \bowtie \hat{r}_{2}=\left\{t_{1} \wedge t_{2} \mid t_{1} \in r_{1}, t_{2} \in r_{2}\right.$ e $t_{1}$ e $t_{2}$ são totais nos atributos comuns a $r_{1}$ e $r_{2} \hat{\}}$

Pode-se também definir a junção externa, como proposta por Codd:

- junção externa: $\hat{r}_{1} \bowtie_{x} \hat{r}_{2}=\left(\hat{r}_{1} \bowtie \hat{r}_{2}\right) \cup \hat{r}_{1} \cup \hat{r}_{2}$

A extensão da álgebra relacional está completa. Finalmente, note que estes operadores realmente estendem a álgebra relacional de Codd, no sentido de que, para as X-relações totais (isto é, sem valores vazios) a definição desses operadores coincide com a definição "clássica", ou seja:

- se $r_{1} \cup r_{2}=r_{3}$ então $\hat{r}_{1} \cup \hat{r}_{2}=\hat{r_{3}}$ 
- se $r_{1}-r_{2}=r_{3}$ então $\hat{r}_{1}-\hat{r}_{2}=\hat{r_{3}}$

- se $r_{1} \subseteq r_{2}$ então $\hat{r}_{1} \subseteq \hat{r}_{2}$

- se $r_{1} \times r_{2}=r_{3}$ então $\hat{r}_{1} \times \hat{r}_{2}=\hat{r_{3}}$

- se $\sigma_{\Theta}\left(r_{1}\right)=r_{2}$ então $\sigma_{\Theta}\left(\hat{r}_{1}\right)=\hat{r}_{2}$

- se $\Pi_{X}\left(r_{1}\right)=r_{2}$ então $\Pi_{X}\left(\hat{r}_{1}\right)=\hat{r}_{2}$

\subsection{Relação Universal}

O modelo relacional utilizando uma única relação, dita relação universal, tem sido objeto de pesquisa há algum tempo [MUV84]. Este modelo permite ao usuário visualizar todo o banco de dados como uma única relação, resultado da junção natural de todas as relações iniciais. O objetivo é facilitar a compreensão e visualização do banco de dados.

A utilização de valores vazios em relações universais foi formalizado no trabalho de Lipski [Lip79]. O trabalho de Lerat e Lispki [LL86] discorre sobre o uso de valores inaplicáveis, e também tem uma forte ligação com o conceito de relação universal. A seguir apresentamos em detalhes estes dois estudos.

\subsubsection{Sistemas de informação}

Uma maneira de representar informação incompleta foi proposta por Lipski [Lip79]. Ele apresenta um novo modelo de dados chamado sistema de informação.

Os sistemas de informação dão ao modelo de relação universal um tratamento mais formal, permitindo também o uso de estruturas mais complexas, como conjuntos, por exemplo. Para a descrição desse modelo, é necessário a definição de uma série de conceitos preliminares.

Um dos componentes básicos de um sistema de informação é um conjunto finito X de objetos. Os objetos são caracterizados através de um conjunto finito I de atributos ou itens. Associado a cada atributo $i \in \mathrm{I}$ existe um conjunto finito $D_{i}$, ou domínio do atributo $\mathrm{I}$, que representa os valores que um objeto $x \in \mathrm{X}$ pode assumir no atributo i. Atributos diferentes podem ter o mesmo domínio.

A caracterização completa de um objeto $x \in \mathrm{X}$ é feita associando-se exatamente um valor do domínio $D_{i}$ a cada atributo i. Entretanto, esse valor nem sempre é conhecido. Por isso, nos sistemas de informação é permitida a associação de vários valores 
do domínio a um atributo do objeto. Esses valores representam os possíveis valores do objeto no atributo. Dessa forma, o valor vazio em um atributo é representado em um sistema de informação associando-se todos os valores do domínio $D_{i}$ ao atributo $i$ do objeto. Note que, nesse caso, somente o valor vazio $n_{A}$ é representado.

A associação de valores aos atributos é feita através de uma função U. Essa função associa, para cada atributo $i \in \mathrm{I}$ e valor $d \in D_{i}$ um conjunto de objetos $\mathrm{U}(i, a) \subseteq \mathrm{X}$ para os quais o atributo $i$ pode assumir o valor $a$. Assim, $\mathrm{X}-\mathrm{U}(i, a)$ é o conjunto de objetos onde se sabe que o atributo $i$ não assume o valor $a$.

Cada objeto possui um valor (conhecido ou não) em um atributo. Este valor pode ser inclusive um conjunto de elementos em $D_{i}$. Ou seja, para todo $x \in \mathrm{X}$ existe pelo menos um elemento $a \in D_{i}$ tal que o valor de $x$ no atributo $i$ é $a$. Se este valor é desconhecido, temos vários $a_{1}, \ldots, a_{n}$ possíveis - no pior caso, todo o domínio $D_{i}$. Esta é uma forma de representar o valor vazio desconhecido $n_{A}$. De qualquer forma, pode-se dizer que, para cada objeto $x \in \mathrm{X}$ existe $a \in D_{i}$ tal que $x \in \mathrm{U}(i, a)$. Portanto, se considerarmos $U\left(\mathrm{U}(i, a) \mid a \in D_{i}\right)$, teremos todos os objetos. Assim, a função $\mathrm{U}$ satisfaz a propriedade:

$$
\mathrm{U}\left(\mathrm{U}(i, a) \mid a \in D_{i}\right)=\mathrm{X}
$$

para cada $i \in \mathrm{I}$.

A partir da função $\mathrm{U}$, pode-se determinar o conjunto $\mathrm{u}(i, a)$ dos objetos onde o atributo $i$ assume com certeza o valor $a$, isto é, aqueles $x \in \mathrm{X}$ que não pertencem a nenhum $\mathrm{U}(i, b)$, para outro atributo $b \neq a$.

$$
\mathrm{u}(i, a)=\mathrm{X}-\cup\left(\mathrm{U}(i, b) \mid b \in D_{i} \wedge a \neq b\right)
$$

Das equações acima concluem-se os seguintes fatos intuitivos:

- $\mathrm{u}(i, a) \subseteq \mathrm{U}(\mathrm{i}, \mathrm{a})$

- $\mathrm{u}(i, a) \cap \mathrm{U}(i, b)=\Phi$

O significado da primeira expressão é que, se sabemos com certeza que o valor de um objeto $x$ no atributo $i$ é $a$, então $x$ está entre os objetos que podem ter o valor $a$ no atributo $i$ (ou, seja, $a$ é um valor possível para $x$ no atributo $i$ ). A segunda expressão significa que, se sabemos com certeza o valor $a$ do objeto $x$ no atributo $i$ então $x$ não pode estar entre os objetos que podem assumir um valor $b$ no atributo $i, b \neq a$. 
Seja $\mathrm{D}$ o conjunto de pares $[i, a]$ onde $i$ é atributo e $a$ um valor do domínio de $i$, isto é, $\mathrm{D}=\left\{[i, a] \mid i \in \mathrm{I}\right.$ e $\left.a \in D_{i}\right\}$, e $\mathcal{P}(\mathrm{X})$ o conjunto de todos os subconjuntos de X (partes de X). Podemos apresentar então a definição formal de um Sistema de Informação:

Um sistema de informação é uma tripla ordenada $\mathcal{I}=\left(\mathrm{X},\left(D_{i}\right)_{i \in I}, \mathrm{U}\right)$ onde

1. X é um conjunto finito de objetos

2. I é um conjunto finito de atributos

3. $D_{i}$ é um conjunto não vazio chamado domínio do atributo $i$

4. U é uma função $\mathrm{U}: \mathrm{D} \rightarrow \mathcal{P}(\mathrm{x})$, tal que para todo $i \in \mathrm{I}, \mathrm{U}\left(\mathrm{U}(i, a) \mid a \in D_{i}\right)=\mathrm{X}$

Pode-se definir também o conjunto de todos os valores que um atributo pode assumir para um objeto $\mathrm{x}$, isto é,

$$
\beta_{i}(x)=\left\{a \in D_{i} \mid x \in \mathrm{U}(i, a)\right\}
$$

Ou seja,

$$
\forall i: x \in \mathrm{U}(i, a) \Leftrightarrow a \in \beta_{i}(x)
$$

Portanto, um sistema de informação pode ser representado por X, $\left(D_{i}\right)_{i \in I}$ e $\left(\beta_{i}\right)_{i \in I}$. Além disso, a definição da função u também pode ser re-escrita em termos de $\beta_{i}$ :

$$
\mathrm{u}(i, a)=\left\{x \in \mathrm{X} \mid \beta_{i}(\mathrm{x})=\{a\}\right\}
$$

Pelo fato de cada objeto possuir um valor no atributo $\mathrm{i}, \beta_{i}(x) \neq \Phi$, para todo $i \in$ $\mathrm{I}, x \in \mathrm{X}$.

Note que uma tupla $\left(\beta_{i}(x)\right)_{i \in I}$ tem uma interpretação muito parecida com as tuplas de relações no modelo relacional. Entretanto, no Sistema de Informação $\beta_{i}(x)$ é normalmente um conjunto de valores dentre os quais um é o valor real de $x$ no atributo $i$, mas não se consegue precisar exatamente qual. Como já comentado, o valor vazio $n_{A}$ pode ser representado tomando-se $\beta_{i}(x)=D_{i}$.

Para ilustrar a definição de Sistema de Informação, apresentamos a seguir um pequeno exemplo. Considere uma ficha médica com os dados: Idade, Peso, Altura. Estão cadastrados Pedro, Maria e Carlos. Então: 
- $\mathrm{X}=\{$ Pedro, Maria, Carlos $\}$

- $\mathrm{I}=\{$ Idade, Peso, Altura $\}$

- $D_{\text {Idade }}=[0,150]$

- $D_{\text {Peso }}=(0,300000)$ (em gramas)

- $D_{\text {Altura }}=(0,220)$ (em centímetros)

- Para Pedro: $\beta_{\text {Idade }}=25, \beta_{\text {Peso }}=\{75000,76000\}, \beta_{\text {Altura }}=170$

- Para Maria: $\beta_{\text {Idade }}=60, \beta_{\text {Peso }}=\{61000,62000\}, \beta_{\text {Altura }}=155$

- Para Carlos: $\beta_{\text {Idade }}=\{27,28\}, \beta_{\text {Peso }}=78000, \beta_{\text {Altura }}=175$

No exemplo, a notação $\beta_{P e s o}=\{61000,62000\}$ significa que o peso deve ser 61000 ou 62000 gramas. Analogamente, $\beta_{\text {Idade }}=\{27,28\}$ indica que a idade deve ser 27 ou 28 anos.

Lipski introduziu também o conceito de extensão de um Sistema de Informação, bastante similar (semanticamente) ao conceito de relação mais informativa de Zaniolo. Dizemos que um sistema de informação $\mathcal{I}_{2}$ é uma extensão de $\mathcal{I}_{1}$ (notação $\mathcal{I}_{1} \leq \mathcal{I}_{2}$ ) se $\mathcal{I}_{1}$ e $\mathcal{I}_{2}$ são definidos sobre os mesmos objetos e atributos e

$$
U_{2}(i, a) \subseteq U_{1}(i, a)
$$

ou, de forma equivalente,

$$
\beta_{i}^{2}(i, a) \subseteq \beta_{i}^{1}(i, a)
$$

A explicação intuitiva dessa definição formal é bem simples: no sistema $\mathcal{I}_{2}$, a informação sobre a caracterização dos objetos é mais completa, no sentido em que temos menos possibilidades e portanto o conjunto $\beta_{i}^{2}(i, a)$ possui menos elementos que $\beta_{i}^{1}(i, a)$. Como $\mathcal{I}_{2}$ se propõe a ser uma extensão de $\mathcal{I}_{1}$, é natural que em $\beta_{i}^{2}(i, a)$ tenhamos somente valores em $\mathcal{I}_{1}$ e portanto, que $\beta_{i}^{2}(i, a) \subseteq \beta_{i}^{1}(i, a)$.

No exemplo apresentado, uma extensão poderia ser:

- X, I, $D_{\text {Idade }}, D_{\text {Peso }}, D_{\text {Altura }}$ não se alteram

- Para Pedro: $\beta_{\text {Idade }}=25, \beta_{\text {Peso }}=75000, \beta_{\text {Altura }}=170$ 
- Para Maria: $\beta_{\text {Idade }}=60, \beta_{\text {Peso }}=61000, \beta_{\text {Altura }}=155$

- Para Carlos: $\beta_{\text {Idade }}=\{27,28\}, \beta_{\text {Peso }}=78000, \beta_{\text {Altura }}=175$

Por fim, Lipski chamou de Sistema de Informação completo o sistema onde para cada atributo $i$ de um objeto $x$ há apenas um valor do domínio, isto é,

- $\mathrm{u}=\mathrm{U}$

- $\mathrm{U}(i, a) \cap \mathrm{U}(i, b)=\Phi$, se $a \neq b$

- $\beta_{i}(x)$ tem apenas um elemento de $D_{i}$, para todo $i \in \mathrm{I}$ e todo $x \in \mathrm{X}$.

Pode-se notar que $\leq$ é uma ordem parcial no conjunto de sistemas de informação com mesmos objetos, atributos e domínios. Além disso, um Sistema de Informação completo é um elemento maximal desse conjunto pois, intuitivamente, nao há como precisar ainda mais a informação de um sistema completo.

\subsubsection{Instâncias universais legais}

O tratamento de valores vazios inaplicáveis foi destacado no trabalho de Lerat e Lipski [LL86]. Para desenvolver um método de avaliação de consultas em bancos de dados que permitem o uso de valores vazios inaplicáveis, foi definido o conceito de instância universal legal, que descrevemos a seguir. Este conceito tem forte relação com o modelo de relação universal, como veremos adiante. Inicialmente apresentamos alguns conceitos auxiliares.

Um esquema $\mathrm{S}$ de um banco de dados é um par $\mathrm{S}=(\mathrm{S} ; \leq)$ onde $\mathrm{S}=\{\mathrm{P}, \mathrm{Q}, \mathrm{R}, \ldots\}$ é um conjunto finito de esquemas de relações e $\leq$ é uma ordenação parcial satisfazendo:

$$
\forall \mathrm{P}, \mathrm{Q} \in \mathrm{S}, \mathrm{P} \leq \mathrm{Q} \Rightarrow \alpha(\mathrm{P}) \subseteq \alpha(\mathrm{Q})
$$

onde $\alpha(\mathrm{P})$ denota o conjunto de atributos em $\mathrm{P}$.

$\mathrm{O}$ esquema $\mathrm{S}$ é simples se não contém esquemas de relações idênticos, isto é, para todo $\mathrm{P}, \mathrm{Q}$ em $\mathrm{S}$, se $\alpha(\mathrm{P})=\alpha(\mathrm{Q})$ então $\mathrm{P}=\mathrm{Q}$.

Uma instância $r$ de um esquema $\mathbf{S}=(\mathrm{S}, \leq)$ é uma aplicação que satisfaz:

1. para cada esquema $P$ de $S, r(P)$ é uma relação onde não existem valores vazios

2. para todo $\mathrm{P}, \mathrm{Q} \in \mathrm{S}, \mathrm{P} \leq \mathrm{Q} \Rightarrow \Pi_{\alpha(P)} r(\mathrm{Q}) \subseteq r(\mathrm{P})$ 
Em uma relação $r_{l}$ (possivelmente com valores vazios) sobre $U$, o conjunto de todos os atributos é uma instância universal legal no esquema $S=(S, \leq)$ se, para qualquer tupla $t \in r_{l}$, existe $\mathrm{Q} \in \mathrm{S}$ tal que os valores de $t$ nos atributos de $\mathrm{Q}$ são todos não vazios. Intuitivamente, $r_{l}$ é uma instância universal sobre $\mathrm{S}$ se a projeção de $r_{l}$ sobre os esquemas é uma relação sem valores vazios.

O nome "instância universal legal" tem origem na definição da relação universal [MUV84]. A relação universal pode ser entendida como a junção natural das relações de um banco de dados. Em determinadas situações, no entanto, o resultado dessa junção é uma relação com valores vazios (o exemplo seguinte vai ilustrar um desses casos). Então, uma instância universal legal é uma dessas relações com valores vazios resultantes de uma junção de diversas relações, todas sem valores vazios. Daí a exigência de que a projeção nos esquemas apropriados não possua valores vazios.

O exemplo a seguir ilustra as definições apresentadas. Considere a relação $r_{l}($ Nome, Endereço, Nome de solteira):

\begin{tabular}{|l|l|l|}
\multicolumn{3}{c}{$r_{l}$} \\
\hline Nome & Endereço & Nome de solteira \\
\hline \hline Silva & São Paulo & $n_{I}$ \\
Bastos & Rio & Martins \\
\hline
\end{tabular}

Vamos representar a informação contida nessa relação através do esquema $\mathbf{S}=$ $\{\{\mathrm{P}, \mathrm{Q}, \mathrm{R}\}, \leq\}$, onde

- $\mathrm{P}=\{$ Nome, Endereço, Nome de solteira $\}$,

- $\mathrm{Q}=\{$ Nome, Endereço $\} \mathrm{e}$

- $\mathrm{R}=\{$ Nome, Nome de solteira $\}$,

e portanto $\mathrm{Q} \leq \mathrm{P}$.

Pode-se verificar que $r_{l}$ é uma instância universal legal do esquema $\mathbf{S}$, e como $\mathbf{S}$ é um esquema simples, sob a CWA (hipótese do mundo fechado), este problema (a representação de $r_{l}$ no esquema $\mathbf{S}$ ) tem uma única solução, a instância $r$ definida por:

$$
\begin{aligned}
& r(\mathrm{P})=\Pi_{\alpha(P)}\left(r_{l}\right) \\
& \text { - } r(\mathrm{Q})=\Pi_{\alpha(Q)}\left(r_{l}\right) \\
& \text { - } r(\mathrm{R})=\Pi_{\alpha(R)}\left(r_{l}\right)
\end{aligned}
$$




\begin{tabular}{|l|l|l|}
\multicolumn{3}{c}{$r(\mathrm{P})$} \\
\hline Nome & Endereço & Nome de solteira \\
\hline \hline Bastos & Rio & Martins \\
\hline
\end{tabular}

\begin{tabular}{|l|l|}
\multicolumn{1}{c}{$r(\mathrm{Q})$} \\
\hline Nome & Endereço \\
\hline \hline Bastos & Rio \\
Silva & São Paulo \\
\hline
\end{tabular}

\begin{tabular}{|l|l|}
\multicolumn{2}{c}{$r(\mathrm{R})$} \\
\hline Nome & Nome de solteira \\
\hline \hline Bastos & Martins \\
\hline
\end{tabular}

Note que foi utilizado o sequinte resultado: Se adotamos a hipótese do mundo fechado e o esquema é simples, a informação contida em uma instância universal legal

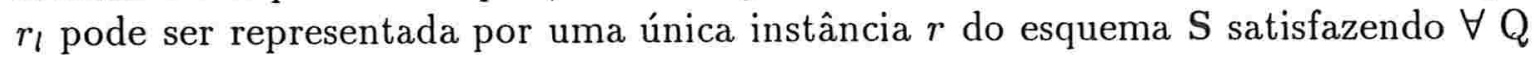
$\in \mathrm{S}, r(\mathrm{Q})=\Pi_{Q}\left(r_{l}\right)$.

Note também que a instância universal $r_{l}$ a seguir também é representada pela mesma instância $r$ :

\begin{tabular}{|l|l|l|}
\multicolumn{3}{c}{$r_{l^{\prime}}$} \\
\hline Nome & Endereço & Nome de solteira \\
\hline \hline Silva & São Paulo & $n_{I}$ \\
Bastos & Rio & Martins \\
Bastos & Rio & $n_{I}$ \\
\hline
\end{tabular}

De qualquer forma, essa decomposição de relações com valores vazios em relações sem valores vazios estabelece um método para avaliação de consultas: cria-se uma linguagem para consultas com valores vazios, traduz-se uma consulta nessa linguagem para uma consulta "normal", isto é, sem valores vazios, e aplica-se essa consulta "normal" às relações obtidas a partir da decomposição da relação original. Esta foi a proposta de Lerat e Lispki para o tratamento do valor vazio inaplicável.

\subsection{Fundamentos de Representações Formais}

As representações formais de relações com valores vazios têm alguns pontos em comum, que Biskup [Bis83] procurou resumir. Estas formalizações têm também uma forte ligação com a lógica de primeira ordem.

O conceito formal de representação da realidade descrito por Biskup se baseia em 3 hipóteses fundamentais: 
- Hipótese do esquema apropriado: Cada esquema de uma relação é apropriado para descrever qualquer estado (aspecto correspondente) da realidade. Cada domínio de atributo é suficientemente rico para descrever todos os eventos possíveis da realidade. Além do mais, para cada evento real possível existe uma tupla que o representa, e cada tupla de uma relação representa um evento real válido.

- Hipótese da informação incompleta: No banco de dados, não se representa diretamente a relação $r$, mas alguns fatos a respeito dessa relação. O significado desses fatos são da forma: "Existem $x_{i_{1}}, \ldots, x_{i_{k}}$ tais que a tupla $\left(b_{1}, \ldots, b_{n}\right)$ é um elemento de $r$ ", onde $b_{i}$ pode ser uma variável quantificada $x_{i}$ se $i \in$ $\left\{i_{1}, \ldots, i_{k}\right\}$ ou um elemento do domínio. Estes fatos podem ser representados e armazenados internamente por uma tupla da forma

$$
\left(\hat{b_{1}}, \ldots, \hat{b_{n}}\right), \text { onde } \hat{b}_{i}= \begin{cases}n_{A} & \text { se } i \in\left\{i_{1}, \ldots, i_{k}\right\} \text { ou } \\ b_{i} & \text { caso contrário }\end{cases}
$$

Nessa representação, o valor vazio $n_{A}$ é apenas uma abreviação para uma variável existencialmente quantificada.

- Hipótese do mundo fechado (uma versão): Se $t$ é uma tupla (sem valores vazios) que não pode ser obtida a partir de uma outra tupla qualquer através da substituição de valores vazios por elementos do domínio, enão $t$ não é um elemento da relação $r$, e portanto o evento real correspondente não ocorre.

Note que qualquer tupla $t$ pode ser obtida a partir da substituição de valores vazios por valores do domínio se armazenamos a tupla $\left(n_{A}, n_{A}, \ldots, n_{A}\right)$ na relação. Nesse caso, estamos assumindo, na verdade, a hipótese do mundo aberto.

A cada tupla é associada uma marca, manipulada através do atributo Status. O valor dessa marca é definido (d) ou possível (p), indicando assim o nível de conhecimento a respeito do evento real correspondente.

A partir desses conceitos apresentados informalmente, pode-se apresentar a definição formal de Representação descrito por Biskup.

Seja $\mathcal{U}$ um conjunto de atributos e $U, V, \ldots, Z$ subconjuntos finitos de $\mathcal{U}$. 0 atributo especial Status não faz parte do conjuto $\mathcal{U}$. Seja $D$ um conjunto infinito de constantes (onde não constam os valores vazios). Chamamos de valores regulares estas constantes não vazias. Os subconjuntos finitos $D_{i}$ de $D$ são chamados de domínios dos atributos. O valor vazio $n_{A}$ não é um elemento de $D$. Seja $D^{\prime}=D \cup\left\{n_{A}\right\}$ o conjunto de valores, $\mathrm{d}$ e $\mathrm{p}$ duas marcas. Usamos a seguinte notação: 
- $D^{*}=D^{\prime} \cup\{\mathbf{d}, \mathbf{p}\}$

- $X^{*}=\mathrm{X} \cup\{$ Status $\}$

Uma tupla interna, ou simplesmente tupla é uma sequência finita de valores (constantes ou vazios) com uma marca, onde as posições são identificadas pelos atributos. Pode-se ver uma tupla interna como uma função:

$$
t: X^{*} \rightarrow D^{*}
$$

isto é, a cada atributo em $X^{*}$ é associado um valor em $D^{*}$, sendo que, obrigatoriamente, $t($ Status $) \in\{\mathbf{d}, \mathbf{p}\}$. Chamamos de esquema o conjunto de atributos $\mathrm{X}$ da tupla $t$.

Uma relação interna é um conjunto finito de tuplas com o mesmo esquema. O esquema de uma relação $r$ é definido da forma:

- $\Phi$, se $r=\Phi$

- $X$, se $r \neq \Phi$ e todas as tuplas em $r$ têm esquema $X$

O exemplo a seguir ilustra os conceitos apresentados:

\begin{tabular}{|c|c|c|}
\hline$A$ & $B$ & Status \\
\hline \hline $\mathrm{a}$ & $n_{A}$ & $\mathrm{~d}$ \\
$\mathrm{~b}$ & $\mathrm{c}$ & $\mathrm{p}$ \\
\hline
\end{tabular}

Nesse caso, o esquema de $r$ é $\{A, B\}$, o domínio pode ser $D=\{\mathrm{a}, \mathrm{b}, \mathrm{c}, \mathrm{d}, \mathrm{e}\}$ para todos os atributos e as tuplas são:

- $t_{1}(A)=\mathrm{a}, t_{1}(B)=n_{A}, t_{1}($ Status $)=\mathrm{d}$

- $t_{2}(A)=\mathrm{b}, t_{1}(B)=\mathrm{c}, t_{2}($ Status $)=\mathrm{p}$

O domínio regular $D_{\text {reg }}(t)$ de uma tupla $t: X^{*} \rightarrow D^{*}$ é o conjunto de todos os valores regulares da tupla $t$. O domínio regular de uma relação $r$ é a união de todos os domínios regulares das tuplas em $r$.

Uma tupla-modelo (também chamada de tupla, quando não houver confusão com a tupla interna) é uma sequência finita de valores regulares (isto é, sem valores vazios nem marcas), onde as posições são novamente identificadas por atributos. Assim como as tuplas internas, as tuplas-modelo podem ser vistas como uma função: 


$$
t: X \rightarrow D
$$

Uma relação modelo é, analogamente, um conjunto finito de tuplas-modelo com o mesmo esquema $X$. Usamos a notação $r, s, t$ para relações internas e $r_{m}, s_{m}, t_{m}$ para relações modelo.

Um modelo $\left[D_{i}, r_{m}\right]$ é um par onde $D_{i}$ é um subconjunto do domínio $D$ e $r_{m}$ é uma relação modelo. A interpretação de modelo é a mesma da lógica formal. Por simplicidade de uso, assumimos que todos os atributos da relação $r_{m}$ possuem o mesmo domínio.

As relações internas são vistas como o conhecimento incompleto que temos a respeito de um modelo $\left[D_{i}, r_{m}\right]$, que reflete o estado atual do mundo real. A conexão entre relações internas e modelos é feito a partir das seguintes definições:

Seja $t: X^{*} \rightarrow D^{*}$ uma tupla interna. Chamamos de substituição de vazios a função sub $: t_{n_{A}} \rightarrow D$ que associa a cada valor vazio em $t$ um valor regular em $D$. Podemos obter então a tupla modelo $\operatorname{ext}(t, s u b)$ tal que:

$$
\operatorname{ext}(t, s u b)= \begin{cases}t(A) & \text { se } t(A) \in D \\ \operatorname{sub}(A) & \text { se } t(A)=n_{A}\end{cases}
$$

Cada tupla interna determina uma série de tuplas-modelo, uma para cada substituição de vazios possível. Seja П o conjunto (finito) de todas estas substituições. Definimos então o conjunto de todas as extensões possíveis de uma tupla interna $\operatorname{ext}(t, \Pi)=\{\operatorname{ext}(t, s u b) \mid s u b \in \Pi\}$.

Definimos também abreviações para as partes definidas e possíveis, respectivamente, de uma relação interna:

- $r^{d}=\{t \mid t \in r$ e $t($ Status $)=\mathrm{d}\}$

- $r^{p}=\{t \mid t \in r$ e $t($ Status $)=\mathbf{p}\}$

Finalmente, podemos definir o conceito formal de Representação: Seja $r$ uma relação interna e $\left[D_{i}, r_{m}\right]$ um modelo. Dizemos que $r$ representa $r_{m}$ se e somente se existe uma relação interna $s$ tal que $r^{d} \subseteq s \subseteq r$, e tal que para toda $t \in s$ existe um conjunto não vazio de substituições $\Pi_{t}$ tal que

$$
\cup_{t \in s} \operatorname{ext}\left(t, \Pi_{t}\right)=r_{m}
$$

A inclusão $r^{d} \subseteq s$ significa que todas as tuplas definidas representam fatos verdadeiros no modelo. A inclusão $s \subseteq r$ significa que não se pode tomar tuplas que 
não tenham ligação com a relação $r$ para representar $r_{m}$. Além disso, a expressão $\cup_{t \in s} \operatorname{ext}\left(t, \Pi_{t}\right)=r_{m}$ significa que todas as tuplas em $r_{m}$ podem ser obtidas a partir de $r$ com algum tipo de substituição de valores vazios.

$\mathrm{O}$ exemplo seguinte mostra que um modelo pode ter várias representações possíveis:

Seja $D=\{\mathrm{a}, \mathrm{b}, \mathrm{c}\}$ e $\left[D, r_{m}\right]$ como na figura:

\begin{tabular}{|c|c|}
\multicolumn{2}{c}{$r_{m}$} \\
\hline$A$ & $B$ \\
\hline \hline $\mathrm{a}$ & $\mathrm{a}$ \\
$\mathrm{a}$ & $\mathrm{b}$ \\
$\mathrm{b}$ & $\mathrm{b}$ \\
\hline
\end{tabular}

Temos as seguintes representações possíveis para $r$ :

\begin{tabular}{|c|c|c|}
\multicolumn{4}{c}{$r_{1}$} \\
\hline$A$ & $B$ & Status \\
\hline \hline $\mathrm{a}$ & $n_{A}$ & $\mathrm{~d}$ \\
$\mathrm{~b}$ & $\mathrm{~b}$ & $\mathrm{~d}$ \\
\hline
\end{tabular}

\begin{tabular}{|c|c|c|}
\multicolumn{4}{c}{$r_{2}$} \\
\hline$A$ & $B$ & Status \\
\hline \hline $\mathrm{a}$ & $\mathrm{c}$ & $\mathrm{p}$ \\
$n_{A}$ & $n_{A}$ & $\mathrm{~d}$ \\
$\mathrm{~b}$ & $\mathrm{~b}$ & $\mathrm{~d}$ \\
\hline
\end{tabular}

\begin{tabular}{|c|c|c|}
\multicolumn{3}{c}{$r_{3}$} \\
\hline$A$ & $B$ & Status \\
\hline \hline$n_{A}$ & $n_{A}$ & $\mathbf{p}$ \\
\hline
\end{tabular}

Onde temos, para as representações descritas:

- $r_{1}$ :

$$
\begin{aligned}
& -s=r_{1}, \\
& -\Pi_{\left(a, n_{A}, d\right)}=\left\{s u b_{1}, s u b_{2}\right\} \\
& -s u b_{1}(B)=\mathrm{a} \\
& -s u b_{2}(B)=\mathrm{b} \\
& -\Pi_{(a, b, d)}=\Phi
\end{aligned}
$$

- $r_{2}$ :

$$
\begin{aligned}
& -s=\left\{\left(n_{A}, n_{A}, \mathrm{~d}\right),(\mathrm{b}, \mathrm{b}, \mathrm{d})\right\} \\
& -\Pi_{\left(n_{A}, n_{A}, d\right)}=\left\{s u b_{1}, s u b_{2}\right\} \\
& -s u b_{1}(A)=\mathrm{a}, \operatorname{sub}_{1}(B)=\mathrm{a}
\end{aligned}
$$




$$
\begin{aligned}
& -s u b_{2}(A)=\mathrm{a}, s u b_{2}(B)=\mathrm{b} \\
& -\Pi_{(a, b, d)}=\Phi
\end{aligned}
$$

- $r_{3}:$

$$
\begin{aligned}
& -s=r_{3} \\
& -\Pi_{\left(n_{A}, n_{A}, p\right)}=\left\{s u b_{1}, s u b_{2}, s u b_{3}\right\} \\
& -s u b_{1}(A)=\mathrm{a}, s_{u} b_{1}(B)=\mathrm{a} \\
& -s_{u} b_{2}(A)=\mathrm{a}, \operatorname{sub}_{2}(B)=\mathrm{b} \\
& -\operatorname{sub}_{3}(A)=\mathrm{b}, \operatorname{sub}_{2}(B)=\mathrm{b}
\end{aligned}
$$

Assim como um modelo pode ter várias relações internas que o representem, uma relação interna pode representar vários modelos diferentes, de acordo com a substituição de valores vazios escolhida. Podemos então definir a quantidade de informação de uma relação interna $r$ :

$$
I_{r}=\left\{\left[D, r_{m}\right] \mid r \text { representa }\left[D, r_{m}\right]\right\}
$$

Quanto menor for o conjunto $I_{r}$, melhor será a informação incompleta que possuímos. No melhor caso, $I_{r}$ terá apenas um elemento, e a relação $r$ só terá tuplas definidas sem valores vazios.

Pode-se então comparar duas relações $r$ e $s$ quanto à quantidade e qualidade de informação. Dizemos que:

- $s$ é mais fraca que $r$ se $I_{r} \subseteq I_{s}$

- $s$ é equivalente a $r$ se $I_{r}=I_{s}$

- $s$ é estritamente mais fraca que $r$ se $I_{r} \subseteq I_{s}$ e $I_{r} \neq I_{s}$

Note que esta comparação entre quantidade de informação é muito similar à comparação entre quantidade de informação em relações definida por Zaniolo [Zan84].

Neste trabalho aparece um conceito muito importante quando se trabalha com relações com valores vazios, e que merece ser destacado: As relações com valores vazios representam na verdade diversas possibilidades no mundo real, uma para cada possível substituição do valor vazio $n_{A}$ por elementos do domínio. Este conceito tem sido utilizado por diversos autores, conforme veremos nas seções a seguir. 


\subsection{V-tabelas e C-tabelas}

A definição de Sistemas de Representação feita por Imielinski e Lipski [IL84] determina as condições mínimas que uma extensão do modelo relacional para tratamento de valores vazios deve satisfazer. Além disso, permite comparar diferentes propostas de extensão do modelo em relação à correção semântica dos operadores relacionais.

Em [IL84], temos também a definição de duas extensões ao modelo relacional, as V-tabelas e as C-tabelas, que são comparadas com as extensões já conhecidas.

Um conceito básico a ser definido antes de se apresentar os sistemas de representação é o de "multirelação". Em [IL84] temos diversas definições de "multiobjetos" que são, basicamente, sequências de objetos. Uma multirelação no esquema $\left[R_{1}, \ldots, R_{n}\right]$ é uma sequência de relações $\left[r_{1}, \ldots, r_{n}\right]$ tal que cada $r_{i}$ segue o esquema $R_{i}$. A notação adotada é $r, s$, para relações e $\mathbf{r}, \mathbf{s}$ para multirelações. Em geral, sequências de objetos (ou multiobjetos) são representados por caracteres em negrito. O esquema, ou tipo de uma multirelação $\mathrm{r}$ é a sequência $\left[R_{1}, \ldots, R_{n}\right]$ e é representado por $\alpha(\mathbf{r})$. O conjunto de todas as multirelações do tipo $\left[R_{1}, \ldots, R_{n}\right]$ é representado por $\mathcal{R}\left[R_{1}, \ldots R_{n}\right]$. Um conjunto $\mathcal{X}$ de multirelações é homogêneo se $\alpha(\mathbf{r})=\alpha(\mathrm{s})$, para todo $\mathrm{r}, \mathrm{s}$ em $\mathcal{X}$. A classe de todos os conjuntos homogêneos de conjuntos de multirelações é representada por $\mathcal{I}$.

Uma expressão multirelacional $\Omega$ é uma sequência $\mathbf{f}=\left[f_{1}, \ldots, f_{k}\right]$ onde $\Omega$ é um subconjunto dos operadores relacionais usuais (união, diferença, projeção,...) e $f_{i} \in$ $\Omega$, para $\mathrm{i}=1, \ldots, \mathrm{k}$.

Uma multitabela é uma "multirelação generalizada", onde as tuplas podem conter valores vazios. As mesmas notações usadas para multirelações são usadas para multitabelas.

Um sistema de representação é uma tripla $(\mathcal{T}, \operatorname{Rep}, \Omega)$, onde

- $\mathcal{T}$ é um conjunto de multitabelas

- Rep é uma aplicação $\operatorname{Rep}: \mathcal{T} \rightarrow \mathcal{I}$, a ser detalhada posteriormente

- $\Omega$ é uma expressão multirelacional

O significado do mapeamento Rep é o seguinte: para cada multitabela T, $\operatorname{Re} p(\mathbf{T})$ é o conjunto das multirelações que podem ser obtidas a partir de $\mathbf{T}$ substituindo valores vazios por valores do domínio.

Uma definição natural de uma função $\mathrm{f}(\mathrm{T})$, onde $\mathrm{f}$ é uma $\Omega$-expressão (isto é, composições de operadores relacionais em $\Omega$ ) pode ser criada se imaginamos que $\mathbf{T}$ 
representa a informação incompleta que possuímos a respeito de alguma multirelação $\mathbf{r} \in \operatorname{Rep}(\mathbf{T})$. A situação ideal seria que existisse a mesma relação entre $\mathbf{f}(\mathbf{T})$ e $\mathbf{f}(\mathbf{r})$, de modo que $\mathbf{f}(\operatorname{Rep}(\mathbf{T}))=\operatorname{Rep}(\mathbf{f}(\mathbf{T}))$.

Infelizmente, na maioria das vezes a estrutura do conjunto $\mathrm{f}(\operatorname{Rep}(\mathrm{T}))$ não é representável por nenhum $\mathbf{U} \in \mathcal{I}$, isto é, não há nenhum $\mathbf{U}$ em $\mathcal{I}$ tal que $\operatorname{Rep}(\mathbf{U})=$ $\mathrm{f}(\operatorname{Rep}(\mathbf{T}))$.

O último conceito que resta a ser definido para que se possa explicar a "condição" que o mapeamento Rep deve satisfazer é o conceito de f-informação em um conjunto de multirelações $\mathcal{X}$, denotado por $\mathcal{X}^{\mathbf{f}}$. Intuitivamente, $\mathcal{X}^{\mathbf{f}}$ é a multirelação maximal $\mathrm{s}$ tal que $\mathbf{s} \subseteq \mathrm{f}\left(\mathrm{r}^{*}\right)$, para todo $\mathrm{r}^{*} \in \mathcal{X}$. A expressão

$$
\mathcal{X}^{\mathrm{f}}=\bigcap \mathrm{f}(\mathrm{X})
$$

traduz essa noção intuitiva apresentada.

Dizemos que um conjunto $\mathcal{X}, \mathcal{Y}$ de multirelações são $\Omega$-equivalentes se $\mathcal{X}^{\mathbf{f}}=\mathcal{Y}^{\mathfrak{f}}$, para todo $\mathrm{f}$ em $\Omega$ (Notação: $\mathcal{X}^{\mathbf{f}} \equiv \Omega \mathcal{Y}^{\mathbf{f}}$ ). Uma multitabela $\mathrm{T} \Omega$-representa $\mathcal{X}$ se $\operatorname{Rep}(\mathrm{T}) \equiv \Omega \mathcal{X}$.

Finalmente podemos completar a definição de sistemas de representação. Uma tripla $(\mathcal{T}, R e p, \Omega)$ é um sistema de representação se, para cada $\Omega$-expressão f e para cada multitabela $\mathbf{T}$ existe uma multitabela $\mathrm{U}$ que $\Omega$-representa $\mathrm{f}(\operatorname{Rep}(\mathrm{T}))$, isto é, $\mathbf{f}(\operatorname{Rep}(\mathbf{T}))$ pode ser definido de modo que $\operatorname{Rep}(\mathbf{f}(\mathbf{T})) \equiv_{\Omega} \operatorname{Rep}(\mathbf{U}) \equiv_{\Omega} \mathbf{f}(\operatorname{Rep}(\mathbf{T}))$.

A partir dessas definições, Imielinski e Lipski provaram que o modelo de tabelas com valores vazios de Codd com sua álgebra relacional estendida é um sistema de representação onde $\Omega=\{$ Projeção, Seleção\}, ou seja, somente esses operadores relacionais funcionam e podem ser compostos para formarem uma expressão semanticamente correta.

Em [IL84] há algumas propostas de novos modelos de dados. A primeira proposta são as V-tabelas, que possuem, ao invés de uma marca para representar valores vazios, símbolos diferentes para valores vazios diferentes. Adquirindo a habilidade de representar que dois valores vazios, apesar de desconhecidos, representam o mesmo valor do domínio, ganha-se poder suficiente para que o sistema de representação correspondente tenha $\Omega=\{$ Projeção, União, Junção e Seleção positiva $\}$, onde entendese por seleção positiva a seleção onde a condição especificada não envolve negações. $\mathrm{O}$ exemplo a seguir ilustra uma V-tabela: 


\begin{tabular}{|l|l|l|}
\hline Nome & Idade & Endereço \\
\hline \hline Carlos & 25 & São Paulo \\
Juca & 24 & $n_{A_{1}}$ \\
Joana & $n_{A_{2}}$ & Rio \\
José & 38 & $n_{A_{1}}$ \\
\hline
\end{tabular}

Uma extensão das V-tabelas, as C-tabelas, permitem um sistema de representação onde $\Omega=\{$ Projeção, União, Junção e Seleção\}, ou seja, pode-se usar qualquer tipo de expressão no operador de seleção. As C-tabelas têm os mesmos valores vazios identificados das V-tabelas, e uma coluna adicional onde se pode escrever uma condição qualquer envolvendo os valores vazios e valores do domínio. O exemplo a seguir ilustra uma C-tabela:

\begin{tabular}{|l|l|l|l|}
\hline Nome & Idade & Endereço & Condição \\
\hline \hline Carlos & 25 & São Paulo & \\
Juca & 24 & $n_{A_{1}}$ & $n_{A_{1}} \neq$ São Paulo \\
Joana & $n_{A_{2}}$ & Rio & $n_{A_{2}} \neq 20$ \\
Joseé & 38 & $n_{A_{1}}$ & $n_{A_{2}} \neq 20 \wedge n_{A_{1}} \neq$ SPaulo \\
\hline
\end{tabular}

Para cada um dos modelos, V-tabelas e C-tabela, temos em [IL84] uma extensão da álgebra relacional correspondente. $\mathrm{O}$ modelo das V-tabelas tem a grande vantagem de que a definição dos operadores relacionais é praticamente idêntica à dos operadores relacionais "usuais". Dessa forma, a nível conceitual (isto é, sem considerações de implementação), as V-tabelas são um modelo de representação simples e bem mais poderoso que as tabelas com valores vazios de Codd.

\subsection{Relações com valores lógicos armazenados}

Gessert [Ges91] propôs um método para o tratamento de informação incompleta ou inaplicável armazenando, para cada atributo de uma tupla de uma relação onde se permita valores vazios o valor lógico associado. Entende-se por valor lógico um dos valores: Verdadeiro, Falso, Possível e Inaplicável da lógica de 4 valores.

Este método se propõe a substituir o método da marca, no qual se indica que o valor armazenado é um valor vazio (aplicável, inaplicável ou desconhecido). 
A idéia básica é criar um "espelho" de cada relação, onde nos atributos que não fazem parte da chave armazena-se um dos valores (V,F,P,I). O exemplo a seguir representa um pequeno cadastro de funcionários com a tabela lógica associada, onde o atributo Cod é a chave da relação

\begin{tabular}{|l|l|l|}
\hline Cod & Nome & Cargo \\
\hline \hline 1011 & Mario & Diretor \\
205 & Lucia & \\
135 & Claudio & Analista \\
\hline
\end{tabular}

\begin{tabular}{|l|l|l|}
\hline Cod & Nome & Cargo \\
\hline \hline 1011 & $\mathrm{~V}$ & $\mathrm{~V}$ \\
205 & $\mathrm{~V}$ & $\mathrm{P}$ \\
135 & $\mathrm{~V}$ & $\mathrm{~V}$ \\
\hline
\end{tabular}

No exemplo, o atributo Cargo na tupla (205, Lucia, -) é um valor vazio aplicável, pois na tabela lógica temos o valor $\mathbf{P}$.

Quando existe um valor vazio, este é representado colocando-se um valor do domínio padrão na tabela de dados e colocando-se o valor lógico correspondente ( $\mathbf{P}$ ou I) na tabela lógica. Segundo Gessert, este método traz várias vantagens conceituais, pelo fato de não ser necessária nenhuma estrutura adicional além das tabelas no modelo relacional. Além disso, os valores lógicos podem ser manipulados normalmente através de comandos SQL, por exemplo. Pode-se ainda fazer cálculos envolvendo tabelas com valores vazios. Pelo método das marcas de Codd, isto era muito mais complicado uma vez que era necessário fazer a distinção entre valores vazios e nãovazios. Nesse novo método, os valores vazios assumem um valor padrão e a distinção é feita somente na tabela lógica.

\subsection{Informação Indefinida}

Para representar informação indefinida e incompleta, Liu e Sunderraman [LS90] introduziram uma extensão do modelo relacional que chamaram de I-tabelas. Em linhas gerais, uma I-tabela é dividida em três componentes básicos:

- a parte "com certeza", que é uma relação como no modelo relacional.

- a parte "indefinida", representada por uma relação onde cada tupla é uma disjunção de 2 ou mais tuplas.

- a parte "possível", que é também uma relação no sentido usual. 
Intuitivamente, a parte "com certeza" representa os fatos que se conhece totalmente; a parte "indefinida" representa fatos que se conhece parcialmente, isto é, sabe-se que um dos fatos representado pelas tuplas na disjunção é verdadeiro (representação de uma disjunção); e a parte "possível" representa fatos que não se conhece, mas possivelmente são verdadeiros. $\mathrm{O}$ exemplo abaixo ilustra uma I-tabela:

\begin{tabular}{|l|l|}
\hline Nome & Idade \\
\hline \hline João & 25 \\
\hline José & 30 \\
\hline Paulo & 60 \\
\hline \hline Maria & 35 \\
Maria & 36 \\
\hline \hline José & 30 \\
Marcos & 30 \\
\hline \hline Carlos & 30 \\
\hline
\end{tabular}

A I-tabela acima representa os seguintes fatos:

- João tem 25 anos, José tem 30 e Paulo tem 60 anos.

- Maria tem 35 ou 36; Marcos ou José têm 30 anos

- Carlos possivelmente tem 30 anos

Liu e Sunderraman definiram também o conceito de informação representada em uma I-tabela: basicamente uma I-tabela resume as diversas possibilidades da realidade que se conhece parcialmente. Há uma analogia com as relações com valores vazios pois estas também podem ser encaradas como representando diversas relações, uma para cada possível substituição de valores vazios por elementos do domínio. A I-tabela resume algumas dessas possibilidades, ou melhor, representa restrições sobre possíveis valores assumidos pelos vazios.

Formalmente, o esquema de uma I-tabela é um esquema $R=\left[A_{1}, \ldots, A_{n}\right]$ onde a cada nome de atributo $A_{i}$ está associado um domínio $D_{i}$. Uma tabela é uma tripla $T=\left[T_{D}, T_{I}, T_{P}\right]$ onde

- $T_{D} \subseteq D_{1} \times \ldots \times D_{n}$

(definição usual de relação) 
- $T_{I} \subseteq\left\{c \mid c \in 2^{D_{1} \times \ldots \times D_{n}}\right.$ e $\left.|c|>1\right\}$

$\left(T_{I} \subseteq\right.$ conjuntos de tuplas, onde são excluídos os conjuntos unitários - neste caso não há disjunção)

- $T_{P} \subseteq D_{1} \times \ldots \times D_{n}$

(definição usual de relação)

A partir desses conceitos Liu e Sunderraman reviram todos os operadores da álgebra relacional para atuar sobre I-tabelas. Definiram também o conceito de redundância em I-tabelas e um operador para remoção dessas redundâncias.

Em [LS91], Liu e Sunderraman estenderam o conceito de I-tabelas para permitir a representação de informação indefinida sob forma de disjunções entre tuplas de relações diferentes. Para isso definiram as M-tabelas.

Numa analogia com o modelo relacional, as I-tabelas são as relações e as M-tabelas são conjuntos de relações. É necessário considerar conjuntos de relações justamente para se permitir disjunções entre as tuplas de relaçôes com atributos diferentes.

Por exemplo, considere os fatos representados pela I-tabela no exemplo anterior e mais os seguintes:

- Carlos é o filho de Paulo

- Maria é a filha de Paulo, se Maria tiver 35 anos

O segundo fato pode ser re-escrito na forma "Maria tem 36 anos ou Maria é a filha de Paulo". Estes fatos podem ser representados através da seguinte M-tabela:

Idades
\begin{tabular}{|l|l|l|l|}
\hline Nome & Idade & Nome & Filho \\
\hline \hline João & 25 & & \\
\hline José & 30 & & \\
\hline Paulo & 60 & & \\
\hline Maria & 35 & & \\
Maria & 36 & & \\
\hline José & 30 & & \\
Marcos & 30 & & \\
\hline & & Paulo & Carlos \\
\hline Maria & 36 & Paulo & Maria \\
\hline \hline Carlos & 30 & & \\
\hline
\end{tabular}


Ou seja, é como se fossem concatenadas duas (ou mais) I-tabelas, sendo que as tuplas com dados em mais de uma I-tabela são as "disjunções entre tuplas em relações distintas".

A definição formal das M-tabelas é bastante similar à das I-tabelas, com a diferença de incorporar mais de um esquema e não mais fazer distinção entre tuplas definidas e indefinidas ( $T_{D}$ e $T_{I}$ se fundem num único conjunto). A distinção é feita somente entre as tuplas "possíveis". A álgebra relacional é revista e são definidos os conceitos de redundância e o operador para sua remoção.

Um outro tipo de extensão para incorporar informação indefinida foi proposto por Grant [Gra79] que sugeriu o uso de intervalos de valores ao invés de valores atômicos nas relações. Dessa forma, por exemplo, o fato "João tem entre 30 e 40 anos" pode ser expresso em uma única tupla da relação $r$ (Nome, Idade): (João, /30,40/). Grant não discutiu profundamente a extensão completa do modelo relacional necessária para o uso de intervalos. Comenta apenas que seria necessária uma lógica de 3 valores e apresenta uma definição do operador de junção, praticamente idêntica à definição de junção natural, apenas com a diferença de permitir o uso (e portanto teste de igualdade) entre intervalos. 


\section{Capítulo 5}

\section{Representações lógicas}

A especificação formal de bancos de dados pode ser feita, basicamente, de duas formas: utilizando o formalismo lógico ou o algébrico [Cst87]. No formalismo algébrico, utilizamos os operadores da álgebra relacional para descrever o funcionamento dos bancos de dados. No formalismo lógico, a ferramenta utilizada é a lógica de primeira ordem.

A definição de lógicas multivaloradas foi o primeiro passo para se tratar expressões envolvendo valores vazios. Alguns autores preferem utilizar a lógica de primeira ordem como formalismo para descrever o comportamento dos bancos de dados relacionais ao invés de álgebra relacional e analisar sob esta ótica o problema dos valores vazios.

O trabalho de Gallaire et.al. [GMN84] mostra de forma resumida e bastante completa os vários pontos de integração entre a teoria de bancos de dados e a lógica. Em bancos de dados relacionais, por exemplo, a lógica aparece no cálculo relacional, na definição e verificação de restrições de integridade, além da contribuição já destacada no estudo de valores vazios.

Um dos trabalhos mais completos nesse sentido foi desenvolvido por Reiter [Rei84]. Nesse texto, ele destaca a formalização lógica feita por outros autores com base em teoria de modelos e propõe a teoria de prova como uma ferramenta mais poderosa para descrição da teoria de bancos de dados relacionais usando lógica. 


\subsection{Linguagens Relacionais}

Para formalizar a teoria de bancos de dados relacionais através da lógica, Reiter [Rei84] introduziu o conceito de linguagem relacional. As linguagens relacionais são um tipo particular de linguagens de primeira ordem, possuindo os recursos necessários para a descrição formal de um banco de dados relacional e de consultas submetidas ao mesmo. Uma linguagem relacional $\mathrm{R}$ pode ser definida com um par ( $\mathrm{A}, \mathrm{W})$ onde A é um conjunto de símbolos, denominado alfabeto e $\mathrm{W}$ é um conjunto de expressões sintaticamente corretas (chamadas fórmulas). No alfabeto A teremos:

- um conjunto infinito de variáveis: $x, y, z, \ldots$

- um conjunto (não vazio) finito de constantes: a, b, c, 10, Alice, Beto, ...

- um conjunto (não vazio) finito de predicados $\mathrm{P}, \mathrm{Q}, \mathrm{R}$, Funcionário, ...

A cada predicado está associado um inteiro $\mathrm{m}>0$, o grau ("aridade") do mesmo, que denota o número de argumentos do predicado. Dentre o conjunto de predicados, destacam-se alguns de uso especial:

- o predicado binário de igualdade

- um subconjunto de predicados unários, denominados tipos simples

- sinais de pontuação: $(),, \ldots$

- conectivos lógicos: $\Rightarrow$ (implica), $\wedge(\mathrm{e}), \vee(\mathrm{ou}), \neg$ (não), $\Leftrightarrow$ (equivalente).

- quantificadores: $\exists$ (existe), $\forall$ (qualquer).

Não existem símbolos funcionais no alfabeto A (como é usual na lógica de primeira ordem), pois não são necessários para a formalização do banco de dados e das consultas ao mesmo.

Um termo é uma variável ou uma constante. Se $\mathrm{P}$ é um predicado de grau $\mathrm{m}, \mathrm{e}$ $t_{1}, \ldots, t_{m}$ são termos, $\mathrm{P}\left(t_{1}, \ldots, t_{m}\right)$ é uma fórmula atômica. Dizemos que $\mathrm{P}\left(t_{1}, \ldots, t_{m}\right)$ é uma fórmula atômica básica se os termos $\left(t_{1}, \ldots, t_{m}\right)$ são todos constantes.

$\mathrm{O}$ conjunto $\mathrm{W}$ de fórmulas é definido recursivamente da forma:

1. Uma fórmula atômica é uma fórmula.

2. se $w_{1}$ e $w_{2}$ são fórmulas, então $\left(w_{1} \wedge w_{2}\right),\left(w_{1} \vee w_{2}\right),\left(w_{1} \Rightarrow w_{2}\right),\left(w_{1} \Leftrightarrow w_{2}\right),\left(\neg w_{1}\right)$ são fórmulas. 
3. Se $x$ é uma variável e $w$ é uma fórmula, então $\forall x(w)$ e $\exists x(w)$ também são fórmulas.

Os tipos de uma linguagem relacional (uma analogia com os domínios de atributos e relações) também são definidos de maneira recursiva:

1. Um tipo simples é um tipo

2. Se $T_{1}, \ldots, T_{2}$ são tipos, $\left(T_{1} \wedge T_{2}\right),\left(T_{1} \vee T_{2}\right)$ e $\left(\neg T_{1}\right)$ também são.

\subsection{Semântica de linguagens relacionais}

A semântica de uma linguagem relacional $R=(A, W)$ deve precisar o significado de cada símbolo em A e, a partir desses símbolos, atribuir um valor lógico (Verdadeiro, Falso) às fórmulas em W. Para tanto são necessárias as definições a seguir.

Uma interpretação I de uma linguagem relacional $R=(A, W)$ é uma tripla (D, $\mathrm{K}, \mathrm{E})$ onde

- D é um conjunto finito (não vazio), chamado Domínio de I, tal que as variáveis de A só podem assumir valores em $\mathrm{D}$.

- K é uma aplicação que associa a cada constante em A um elemento de D.

- E é uma aplicação que associa a cada predicado m-ário $\mathrm{P}$ em $\mathrm{A}$, um conjunto de m-tuplas em $D^{m}\left(\mathrm{E}(\mathrm{P}) \subseteq D^{m}\right)$.

Dada uma interpretação $I=(D, K, E)$ de uma linguagem relacional $R=(A, W)$, uma atribuição de valores $\rho$ é uma aplicação de variáveis em $\mathrm{A}$ sobre os elementos do domínio $\mathrm{D}$, isto é, para cada variável $x$ em $\mathrm{A}, \rho(x) \in \mathrm{D}$. A partir dessa atribuição de valores a variáveis, pode-se definir uma atribuição de valores a termos da forma:

- $\rho\left(t_{1}\right)=\rho(x)$, se $t_{1}$ é uma variável $x$

- $\rho\left(t_{1}\right)=\mathrm{K}(\mathrm{c})$, se $t_{1}$ é uma constante c

Define-se então a relação de satisfabilidade $\models_{I, \rho}$ da forma:

1. $\models_{I, \rho} \mathrm{P}\left(t_{1}, \ldots, t_{m}\right)$ se e somente se a m-tupla $\left(\rho\left(t_{1}\right), \ldots, \rho\left(t_{m}\right)\right) \in \mathrm{E}(\mathrm{P})$, para cada fórmula atômica $\mathrm{P}\left(t_{1}, \ldots, t_{m}\right)$ em $\mathrm{W}$ 
2. $\models_{I, \rho} w_{1} \wedge w_{2}$ se e somente se $\models_{I, \rho} w_{1} \mathrm{e} \models_{I, \rho} w_{2}$

3. $\models_{I, \rho} w_{1} \vee w_{2}$ se e somente se $\models_{I, \rho} w_{1}$ ou $\models_{I, \rho} w_{2}$

4. $\models_{I, \rho} \neg w_{1}$ se e somente se não ocorre $\models_{I, \rho} w_{1}$.

5. $\models_{I, \rho} w_{1} \Rightarrow w_{2}$ se e somente se $\models_{I, \rho} \neg w_{1}$ ou $\models_{I, \rho} w_{2}$

$6 . \models_{I, \rho} w_{1} \Leftrightarrow w_{2}$ se e somente se $\models_{I, \rho} w_{1} \Rightarrow w_{2}$ e $\models_{I, \rho} w_{2} \Rightarrow w_{1}$

7. $\models_{I, \rho} \forall x\left(w_{1}\right)$ se e somente se, para todo $\mathrm{d} \in \mathrm{D}, \models_{I, \rho[x / d]} w_{1}$, onde $\rho[x / d]$ é uma atribuição de valores idêntica a $\rho$ com a diferença que atribui à variável $x$ o valor $d$.

8. $\models_{I, \rho} \exists x\left(w_{1}\right)$ se e somente se $\models_{I, \rho} \neg \forall x \neg w_{1}$

A partir da definição de $\models_{I, \rho}$, definimos o valor lógico de uma fórmula em uma interpretação. Dizemos que $\models_{I} w$ se e somente se $\models_{I, \rho} w$, para toda atribuição de valores $\rho$. Nesse caso, $w$ é dita Verdadeira na interpretação I. Uma interpretação I é um modelo da fórmula $w$ se $w$ é verdadeira em I. Dizemos que I é um modelo de um conjunto $W$ de fórmulas se $w$ é verdadeira para toda fórmula $w \in W$.

O exemplo "acadêmico" a seguir ilustra os conceitos apresentados. Seja $R=(A$, W) a linguagem relacional onde

- predicados de R: Professor $(x)$, Disciplina $(x), \operatorname{Aluno}(x), \operatorname{Leciona}(x, y)$, Matriculado $(x, y) \mathrm{e}=(x, y)$ (pode-se usar a notação " $(x=y)$ ")

- tipos simples em R: Professor $(x)$, Disciplina $(x), \operatorname{Aluno}(x)$

- constantes em A: \{Prof.Alberto, Prof.Betina, Prof.Claudio, Alice, Beto, Carlos, Diana, Geometria I, Geometria II, Biologia I, Biologia II\}

São fórmulas dessa linguagem, por exemplo:

- $\forall x$ Professor $(x) \Rightarrow \exists y$ Leciona $(x, y)$

- $\forall x \operatorname{Aluno}(x) \Rightarrow \exists y$ Matriculado $(x, y)$

São tipos nessa linguagem, por exemplo: Professor $(x), \operatorname{Professor}(x) \vee \operatorname{Aluno}(x)$, Aluno $(x), \operatorname{Disciplina}(x)$.

Uma possível interpretação I para essa linguagem é: 
- $\mathrm{D}=\{$ Prof.Alberto, Prof.Betina, Prof.Claudio, Alice, Beto, Carlos, Diana, Geometria I, Geometria II, Biologia I, Biologia II $\}$

- K associa uma constante em A com um elemento do domínio D de mesmo nome.

- As tuplas em E são representadas em forma tabular, como segue:

\begin{tabular}{|l|}
\multicolumn{1}{c|}{ Professor } \\
\hline \hline Prof.Alberto \\
Prof.Betina \\
Prof.Claudio \\
\hline
\end{tabular}

\begin{tabular}{|l|}
\multicolumn{1}{c|}{ Disciplina } \\
\hline \hline Geometria I \\
Geometria II \\
Biologia I \\
Biologia II \\
\hline
\end{tabular}

\begin{tabular}{|l|}
\multicolumn{1}{|c|}{ Aluno } \\
\hline \hline Alice \\
Beto \\
Carlos \\
Diana \\
\hline
\end{tabular}

Leciona

\begin{tabular}{|l|l|}
\hline \hline Prof.Alberto & Geometria I \\
Prof.Alberto & Geometria II \\
Prof.Betina & Biologia I \\
Prof.Claudio & Biologia I \\
Prof.Claudio & Biologia II \\
\hline
\end{tabular}

\begin{tabular}{|l|l|}
\multicolumn{1}{c}{ Matriculado } \\
\hline \hline Alice & Geometria I \\
Alice & Biologia I \\
Beto & Geometria I \\
Carlos & Biologia I \\
Diana & Geometria II \\
Diana & Biologia II \\
\hline
\end{tabular}

\begin{tabular}{|l|l|}
\multicolumn{1}{c|}{$=$} \\
\hline \hline Prof.Alberto & Prof.Alberto \\
Prof.Betina & Prof.Betina \\
Prof.Claudio & Prof.Claudio \\
Alice & Alice \\
Beto & Beto \\
Carlos & Carlos \\
Diana & Diana \\
Geometria I & Geometria I \\
Geometria II & Geometria II \\
Biologia I & Biologia I \\
Biologia II & Biologia II \\
\hline
\end{tabular}

Essa interpretação é um modelo para, por exemplo, as seguintes fórmulas:

- $\forall x \forall y$ (Leciona $(x, y) \Rightarrow \operatorname{Professor}(x) \wedge$ Aluno $(y))$ 
- $\forall x \forall y$ (Matriculado $(x, y) \Rightarrow$ Aluno $(x) \wedge$ Disciplina $(y))$

- $\forall x \exists y(\operatorname{Disciplina}(x) \wedge \operatorname{Professor}(y) \Rightarrow$ Leciona $(x, y))$

- $\forall x \exists y(\operatorname{Disciplina}(y) \wedge \operatorname{Professor}(x) \Rightarrow$ Leciona $(x, y))$

A definição das linguagens relacionais e suas interpretações são úteis para deixar clara a distinção entre símbolos sintáticos (objetos no alfabeto) e objetos do domínio. $\mathrm{Na}$ área de bancos de dados é comum assumir que as tabelas definem uma série de proposições verdadeiras. No exemplo anterior, temos as proposições Professor(Prof.Alberto), ..., Matriculado(Diana, Biologia II), etc. As proposições não definidas (isto é, que não constam nas tabelas) são assumidas como falsas (CWA).

Uma interpretação $I=(D, K, E)$ de uma linguagem relacional $R=(A, W)$ é uma interpretação relacional se:

1. O mapeamento $\mathrm{K}: \mathrm{A} \rightarrow \mathrm{D}$ é uma função injetora e sobrejetora

2. $\mathrm{E}(=)=\{(\mathrm{d}, \mathrm{d}) \mid \mathrm{d} \in \mathrm{D}\}$

Um banco de dados relacional é uma tripla (R,I,IC) onde

1. $\mathrm{R}$ é uma linguagem relacional

2. I é uma interpretação relacional de $\mathrm{R}$

3. IC é um conjunto de fórmulas em $\mathrm{R}$, chamadas de restrições de integridade. Em particular, para todo predicado $\mathrm{m}$-ário $\mathrm{P}$, a menos da igualdade e de tipos simples deve existir em IC uma fórmula do tipo $\forall x_{1} \ldots \forall x_{m} \mathrm{P}\left(x_{1}, \ldots, x_{m}\right) \Rightarrow$ $T_{1}\left(x_{1}\right) \wedge \ldots \wedge T_{m}\left(x_{m}\right)$, onde $T_{i}$ são tipos.

Para cada predicado $\mathrm{P}$, à exceção dos tipos simples, o mapeamento $\mathrm{E}(\mathrm{P})$ é chamado de relação. Quando não houver confusão, pode-se escrever a relação $\mathrm{P}$ para denotar o mapeamento $\mathrm{E}(\mathrm{P})$. As restriçôes de integridade em IC são ditas satisfeitas se I é um modelo para IC.

Podemos notar vários pontos de correspondência entre essa abordagem para a especificação de bancos de dados e as abordagens analisadas no capítulo 4 :

- Os esquemas das relações na álgebra relacional correspondem à aridade dos predicados (a menos dos destacados como tipos ou como igualdade) no formalismo lógico. 
- Os atributos na álgebra relacional correspondem aos predicados destacados como tipos das linguagens relacionais no formalismo lógico.

- Os domínios dos atributos na álgebra relacional correspondem aos elementos associados pelas aplicações E (da interpretação das linguagens relacionais) aos tipos no formalismo lógico.

- As relações na álgebra relacional correspondem à aplicação E associada aos predicados que não são destacados como tipos nem como igualdade. Essa aplicação associa a esses predicados o conjunto de tuplas que é a "relação" no formalismo lógico.

Reiter [Rei84] apontou uma série de problemas da representação através da teoria de modelos. Em particular, o problema dos valores vazios foi analisado (na verdade apenas o valor vazio $n_{A}$ ). Como já foi visto anteriormente, permitir o uso de valores vazios nas relações traz a necessidade de uma lógica multivalorada para avaliar expressões que envolvem valores vazios. Com isso, é necessário reavaliar também os operadores da álgebra relacional.

Segundo Reiter, o uso de lógicas multivaloradas é uma consequência direta e natural da representação através de teoria de modelos dos bancos de dados relacionais, pois os modelos estão intimamente ligados ao conceito lógico de Verdade. Uma vez que dois valores lógicos são suficientes para avaliação de consultas em bancos de dados sem valores vazios, é natural introduzir novos valores lógicos para consultas em interpretações onde é permitido o uso de valores vazios.

\subsection{Teoria de prova}

Uma teoria relacional de uma linguagem relacional $R=(A, W)$ é um subconjunto de fórmulas $\mathrm{T} \subseteq \mathrm{W}$ que satisfazem as seguintes propriedades:

1. Se $c_{1}, \ldots, c_{n}$ são as constantes do alfabeto $\mathrm{A}, \mathrm{T}$ deve conter as fórmulas:

(a) $\forall x\left(x=c_{1} \vee \ldots \vee x=c_{n}\right)$. Esta fórmula, denominada axioma do domínio fechado, traduz o fato de que as constantes do alfabeto são os únicos indivíduos na teoria. Dessa forma, estamos assumindo a CWA.

(b) Uma série de fórmulas do tipo $\neg\left(c_{i}=c_{j}\right)$, para $\mathrm{i}, \mathrm{j}=1 \ldots \mathrm{n}, \mathrm{i}<\mathrm{j}$. Estas fórmulas, denominadas axiomas dos nomes únicos, traduzem o fato de que constantes com nomes distintos representam indivíduos distintos. 
2. $\mathrm{T}$ deve conter os axiomas da igualdade:

(a) Reflexividade: $\forall x(x=x)$

(b) Comutatividade: $\forall x \forall y(x=y \Rightarrow y=x)$

(c) Transitividade: $\forall x \forall y \forall z(x=y \wedge y=z \Rightarrow x=z)$

(d) Princípio da substituição de termos iguais (Leibnitz): Para cada predicado m-ário $\mathrm{P}$ de $\mathrm{A}, \forall x_{1} \ldots \forall x_{m}\left[\mathrm{P}\left(x_{1}, \ldots, x_{m}\right) \wedge x_{1}=y_{1} \wedge \ldots \wedge x_{m}=y_{m} \Rightarrow\right.$ $\left.\mathrm{P}\left(y_{1}, \ldots, y_{m}\right)\right]$.

3. Para algum subconjunto $\Delta \subseteq \mathrm{W}$ de fórmulas atômicas básicas, (sendo que nenhuma das fórmulas em $\Delta$ contém a igualdade), $\Delta \subseteq T$. Estas fórmulas traduzem os fatos especificados pelas relações no banco de dados. Para cada predicado m-ário (a menos da igualdade), definimos um conjunto $C_{P}$ de m-tuplas de contantes $C_{P}=\left\{\left(c_{1}, \ldots, c_{m}\right) \mid \mathrm{P}\left(c_{1}, \ldots, c_{m}\right) \in \Delta\right\}$. O conjunto $\left\{\mathrm{P}\left(c_{1}, \ldots, c_{m}\right)\right.$ $\left.\mid\left(c_{1}, \ldots, c_{m}\right) \in C_{P}\right\}$ é chamado a extensão da relação $\mathrm{P}$ em T. Seja $C_{P}=$ $\left\{\left(c_{1}^{1}, \ldots, c_{m}^{1}\right),\left(c_{1}^{2}, \ldots, c_{m}^{2}\right), \ldots,\left(c_{1}^{s}, \ldots, c_{m}^{s}\right)\right\}$. Então, além das fórmulas em $\Delta$, $\mathrm{T}$ deve conter a seguinte fórmula, chamada axioma do completamento:

$$
\begin{aligned}
\forall x_{1} \ldots \forall x_{m} P\left(x_{1}, \ldots, x_{m}\right) \Rightarrow \quad & \left(x_{1}=c_{1}^{1} \wedge \ldots \wedge x_{m}=c_{m}^{1}\right) \vee \\
& \left(x_{1}=c_{1}^{2} \wedge \ldots \wedge x_{m}=c_{m}^{2}\right) \vee \ldots \vee \\
& \left(x_{1}=c_{1}^{s} \wedge \ldots \wedge x_{m}=c_{m}^{s}\right)
\end{aligned}
$$

Se $C_{P}=\{\}$, então a extensão de $\mathrm{P}$ em T é vazia, e o axioma do completamento fica da forma $\forall x_{1} \ldots \forall x_{m} \neg P\left(x_{1}, \ldots, x_{m}\right) O$ axioma do completamento traduz o fato de que somente as tuplas pertencentes à relação $\mathrm{P}$ são fatos verdadeiros, isto é, a fórmula $\neg P\left(d_{1}, \ldots, d_{m}\right)$ deve ser verdadeira, se a tupla $\left(d_{1}, \ldots, d_{m}\right)$ não pertence à relação $\mathrm{P}$. Novamente estamos assumindo a hipótese do mundo fechado (CWA).

4. Somente as fórmulas especificadas nos itens anteriores estão em T, isto é, T não contém mais nenhuma fórmula.

No exemplo "acadêmico", a teoria T deve conter os axiomas:

1. domínio fechado: $\forall x(x=$ Prof.Alberto $) \vee(x=$ Prof.Betina $) \vee \ldots \vee$ $(x=$ Biologia I $) \vee(x=$ Biologia II $)$

2. nomes únicos: $\neg$ (Prof.Alberto=Prof.Betina $), \neg($ Biologia $I=$ Alice $), \ldots$, $\neg($ Diana $=$ Biologia II $)$

3. axiomas da igualdade 
4. fatos atômicos em $\Delta$ : Professor(Prof.Alberto), .., Disciplina(Biologia I), .., Matriculado(Diana,Biologia II)

5. axiomas de completamento:

- $\forall x$ (Professor $(x) \Rightarrow(x=$ Prof.Alberto $) \vee(x=$ Prof.Betina $) \vee$ ( $x=$ Prof.Claudio)

- $\forall x(\operatorname{Disciplina}(x) \Rightarrow(x=$ Geometria I $) \vee(x=$ Geometria II $) \vee$ $(x=$ Biologia I $) \vee(x=$ Biologia II $)$

- $\forall x(\operatorname{Aluno}(x) \Rightarrow(x=$ Alice $) \vee(x=$ Beto $) \vee(x=$ Carlos $) \vee(x=$ Diana $))$

- $\forall x \forall y$ (Leciona $(x, y) \Rightarrow(x=$ Prof.Alberto $) \wedge y=$ Geometria I) $\vee$ $(x=$ Prof.Alberto $\wedge y=$ Geometria II $) \vee$ $(x=$ Prof.Betina $\wedge y=$ Biologia I $) \vee$ $(x=$ Prof.Claudio $\wedge y=$ Biologia I $) \vee$ $(x=$ Prof.Claudio $\wedge y=$ Biologia II $))$

- $\forall x \forall y$ (Matriculado $(x, y) \Rightarrow(x=$ Alice $\wedge y=$ Geometria I) $\vee$ $(x=$ Alice $\wedge y=$ Biologia I $) \vee$ $(x=$ Beto $\wedge y=$ Geometria I $) \vee$ $(x=$ Carlos $\wedge y=$ Biologia I $) \vee$ $(x=$ Diana $\wedge y=$ Geometria II $) \vee$ $(x=$ Diana $\wedge y=$ Biologia II $))$

Reiter provou a equivalência entre interpretações e teorias relacionais mostrando que cada teoria relacional possui uma única interpretação relacional e vice-versa, isto é, para cada interpretação relacional pode-se contruir uma teoria relacional correspondente. Dessa forma, pode-se definir um banco de dados relacional através de teorias relacionais.

Um banco de dados relacional é uma tripla $(\mathrm{R}, \mathrm{T}, \mathrm{IC})$ onde $\mathrm{R}$ e IC são como anteriormente e T é uma teoria relacional. As restrições de integridade em IC são ditas satisfeitas se para cada $w \in \mathrm{IC}, \mathrm{T} \vdash w$, ou seja, pode-se construir uma prova de $w$ a partir dos axiomas em $\mathrm{T}$.

Reiter apresentou também extensões da teoria relacional para representar fatos disjuntivos e valores vazios. Neste texto consideramos apenas a extensão para valores vazios. Basicamente, deve-se considerar um conjunto extra de constantes (valores vazios) $\mathrm{N}=\left\{n_{A_{1}}, \ldots, n_{A_{r}}\right\}$. As constantes do alfabeto A são separadas em dois grupos disjuntos: vazios e não vazios. São necessários vários símbolos $n_{A_{i}}$ pois, para cada fato 
(tupla) envolvendo valores vazios, deve ser usado um símbolo diferente, caso contrário a teoria de prova entende todos os valores vazios como uma única constante, e podese obter resultados indesejáveis. Por exemplo, podemos incluir no banco de dados os fatos:

- Alice está matriculada em mais uma disciplina, mas não se sabe qual

- Beto está matriculado em um curso lecionado pelo Prof.Claudio

correspondentes às fórmulas:

- Matriculado(Alice, $\left.n_{A}\right)$.

- Matriculado(Beto, $\left.n_{A}\right) \wedge$ Leciona(Prof.Claudio, $\left.n_{A}\right)$.

Se incluimos essas fórmulas, através da teoria de prova construída conclui-se que Alice está matriculada no mesmo curso que Beto, e que este é lecionado pelo Prof.Claudio, mas não era essa a intenção. A solução é identificar cada valor vazio como uma nova constante (ou seja, utilizamos o mesmo conceito de valores vazios identificados descrito no capítulo 3 ), sempre que se insere um novo fato independente. No caso, devemos incluir as fórmulas:

- Matriculado(Alice, $n_{A_{1}}$ )

- Matriculado(Beto, $\left.n_{A_{2}}\right) \wedge$ Leciona(Prof.Claudio, $\left.n_{A_{2}}\right)$

Os valores vazios não devem constar nos axiomas de nome únicos, por razões óbvias. Como não se sabe o valor real que um valor vazio $n_{A}$ representa, não se deve afirmar $n_{A} \neq \mathrm{c}$, para nenhuma constante (não vazia) c. Em casos particulares, no entanto, pode-se incluir fórmulas desse tipo. Por exemplo, se temos a informação: "Beto está matriculado em mais um curso, não se sabe qual, mas não pode ser Biologia II", incluimos as fórmulas Matriculado(Beto, $\left.n_{A_{1}}\right)$ e $\neg\left(n_{A_{1}}=\right.$ Biologia II).

Uma teoria relacional com valores vazios sobre uma linguagem relacional $R=(A, W)$, onde $\mathrm{A}=\mathrm{C} \cup \mathrm{N}$, é um conjunto de fórmulas que satisfazem as propriedades:

1. $\mathrm{T}$ contém os axiomas de domínio fechado: $\forall x\left(x=c_{1} \vee \ldots \vee x=c_{n} \vee x=\right.$ $\left.n_{A_{1}} \vee \ldots \vee x=n_{A_{r}}\right)$.

T contém os axiomas de nome único: $\neg\left(c_{i}=c_{j}\right)$, para $\mathrm{i}, \mathrm{j}=1, \ldots, \mathrm{n}, \mathrm{i}<\mathrm{j}$.

$\mathrm{T}$ pode conter uma ou mais desigualdades da forma: 
- $\neg\left(n_{A_{\mathrm{i}}}=c_{j}\right)$, para $1 \leq \mathrm{i} \leq \mathrm{r}, 1 \leq \mathrm{j} \leq \mathrm{n}$.

- $\neg\left(n_{A_{i}}=n_{A_{j}}\right)$, para $\mathrm{i}, \mathrm{j}=1, \ldots, \mathrm{r}, \mathrm{i}<\mathrm{j}$.

2. $\mathrm{T}$ contém os axiomas da igualdade usuais.

3. Para algum subconjunto $\Delta \subseteq \mathrm{W}$ de fórmulas atômicas básicas, $\Delta \subseteq T$. Para cada predicado m-ário $\mathrm{P}$ (a menos da igualdade), definimos um conjunto $K_{P}$ de m-tuplas de contantes de $\mathrm{C} \cup \mathrm{N}$ da forma $K_{P}=\left\{\left(k_{1}, \ldots, k_{m}\right) \mid \mathrm{P}\left(k_{1}, \ldots, k_{m}\right)\right.$ $\in \Delta\}\left(k_{i}\right.$ pode ser $c_{j}$ ou $\left.n_{A_{j}}\right)$. Seja $K_{P}=\left\{\left(k_{1}^{1}, \ldots, k_{m}^{1}\right), \ldots,\left(k_{1}^{s}, \ldots, k_{m}^{s}\right)\right\}$. Então, além das fórmulas em $\Delta, \mathrm{T}$ deve conter a seguinte fórmula, chamada axioma do completamento:

$$
\begin{aligned}
\forall x_{1} \ldots \forall x_{m} P\left(x_{1}, \ldots, x_{m}\right) \Rightarrow \quad & \left(x_{1}=k_{1}^{1} \wedge \ldots \wedge x_{m}=k_{m}^{1}\right) \vee \\
& \left(x_{1}=k_{1}^{2} \wedge \ldots \wedge x_{m}=k_{m}^{2}\right) \vee \ldots \vee \\
& \left(x_{1}=k_{1}^{s} \wedge \ldots \wedge x_{m}=k_{m}^{s}\right)
\end{aligned}
$$

Se $K_{P}=\{\}$, então o axioma do completamento fica da forma $\forall x_{1} \ldots \forall x_{m} \neg P\left(x_{1}, \ldots, x_{m}\right)$

4. Somente as fórmulas especificadas nos itens anteriores estão em T.

A definição de bancos de dados relacionais com valores vazios é idêntica à de bancos de dados relacionais usando uma teoria relacional com valores vazios ao invés de teoria relacional. A distinção entre constantes não vazias e valores vazios é feita através da omissão dos axiomas de nomes únicos para os valores vazios, justamente para exprimir o fato de que não se possui nenhuma informação sobre a identidade dos mesmos.

\subsection{Semântica de partição}

Utilizando teoria de modelos para representação de bancos de dados relacionais, Laurent e Spyratos [LS88] desenvolveram um algoritmo para cálculo de consultas em bancos de dados com informações incompletas e dependências funcionais. Entende-se por informação incompleta, nesse caso, fatos disjuntivos.

Reiter [Rei84] já havia notado que a teoria de modelos poderia ser uma forma elegante de se formalizar e tratar este problema. No exemplo do banco de dados "acadêmico", o problema aparece se tentamos representar "Beto está matriculado 
em Biologia I ou Biologia II, mas não se sabe qual". Através da teoria de modelos, representam-se esses fatos considerando duas interpretações, uma onde temos Matriculado(Beto, Biologia I) e outra onde temos Matriculado(Beto, Biologia II).

Com a formalização desse conceito intuitivo apresentado por Reiter, Laurent e Spyratos definiram dois tipos de tuplas em um banco de dados relacional lógico:

- $t$ é dita certa se $t$ é verdadeira em todas as interpretações consideradas

- $t$ é dita possível se $t$ é verdadeira em alguma das interpretações consideradas

No exemplo apresentado a tupla Matriculado(Alice, Geometria I) seria certa, e a tupla Matriculado(Beto, Biologia I) seria possível

A partir dessa definição, os autores desenvolveram um algoritmo para avaliação de consultas que, basicamente, constrói um modelo especial para o banco de dados, que contém as informações necessárias para que se calculem as tuplas certas e possíveis como resposta a uma consulta.

É interessante destacar que a lógica é um formalismo elegante para se tratar o problema de valores vazios, seja através da teoria de modelos ou da teoria de prova. No entanto, as representações lógicas e algébricas são equivalentes, e se confrontam com o mesmo tipo de problema decorrente da utilização de valores vazios: enquanto na representação algébrica a preocupação é com o efeito dos operadores relacionais sobre as diversas possibilidades representadas por um banco de dados com valores vazios, na teoria de modelos procura-se verificar a validade de uma fórmula nos diversos modelos de um banco de dados com valores vazios.

A representação através de teoria de prova parece ser mais vantajosa nesse sentido, mas esta vantagem é um pouco ilusória [HPRV 89], pois sugere-se que a avaliação de consultas seja feita como prova de teoremas. Um provador de teoremas que utiliza um método genérico (p.ex. resolução) não possui a eficiência necessária para ser utilizado em um banco de dados de proporções razoáveis. Consequentemente é necessário desenvolver algoritmos específicos para consultas nessa representação (no capítulo seguinte veremos alguns). Estes algoritmos nem sempre devolvem todas as respostas a um dada consulta.

Além disso, o tratamento de valores vazios através da lógica não leva em consideração os diversos tipos diferentes de valor vazio. $O$ valor vazio $n_{I}$ tem uma interpretação bem diferente de $n_{A}$, e portanto, a rigor a teoria deveria ser revista para tratar adequadamente os demais tipos de valores vazios. 


\section{Capítulo 6}

\section{Consultas}

$\mathrm{O}$ acesso a bancos de dados relacionais para obtenção de informação pode ser descrito através dos operadores da álgebra relacional. Portanto, a descrição de consultas nesses bancos está intimamente ligada com o funcionamento desses operadores.

No capítulo 4 apresentamos diversas extensões do modelo relacional. No presente capítulo procuramos resumir os problemas encontrados nos operadores da álgebra relacional em várias dessas extensões e analisar o motivo de surgirem tais problemas. Apresentamos ainda algumas idéias adicionais sobre a semântica desses operadores.

No capítulo 5 vimos representações lógicas de bancos de dados relacionais e observamos a necessidade de algoritmos eficientes para avaliação de consultas quando via teoria de prova. Apresentamos também no presente capítulo alguns algoritmos para consultas via teoria de prova.

\subsection{Seleção e Junção}

Os operadores de Seleção e Junção são os que mais sofrem modificações com a introdução de valores vazios nas relações. A seleção é modificada porque utiliza expressões de comparação entre elementos do domínio (que agora pode ter valores vazios). A junção, por ser construída a partir da seleção, também precisa ser modificada. Alguns autores notaram que com o uso de valores vazios pode-se definir um novo tipo de junção, mais abrangente, que Codd [Codd79] chamou de junção externa.

A modificação na seleção constitui-se basicamente em acrescentar mais um parâmetro à operação: além de especificar a expressão de comparação, deve-se especificar também o valor lógico da expressão. Esta idéia foi usada por Codd - que definiu as seleções 
verdadeira e a possível (Cap.4); por Zaniolo, que considerou apenas a versão verdadeira (Cap.4); e por Gessert, que ao estender os comandos de SQL, acrescentou à cláusula where uma sintaxe para especificar valores lógicos (Cap.3).

A junção ganhou sua versão "externa" a partir da idéia proposta em [LP76], onde Lacroix e Pirotte propõem uma junção "generalizada" cujo objetivo é possibilitar a recuperação dos operandos a partir do resultado final da junção. No capítulo 4 foram descritas as adaptações de Codd e Zaniolo para essa idéia. Em [LP76] temos ainda a definição de operadores de junção não simétricos, onde apenas um dos operandos pode ser extraído do resultado final. A definição desses operadores é a seguinte : sejam $r(\mathrm{R}), s(\mathrm{~S})$ duas relações e $R_{1}=\mathrm{R}-(\mathrm{R} \cap \mathrm{S}), R_{2}=\mathrm{S}-(\mathrm{R} \cap \mathrm{S})$. Então:

$$
\begin{aligned}
& r+\bowtie s=r \bowtie s \cup\left(\left(r-\Pi_{R}(r \bowtie s)\right) \times\left\{S_{1}: n_{A}\right\}\right) \\
& \text { - } r \bowtie_{+} s=r \bowtie s \cup\left(\left(s-\Pi_{S}(r \bowtie s)\right) \times\left\{R_{1}: n_{A}\right\}\right)
\end{aligned}
$$

onde $\left\{X: n_{A}\right\}$ representa uma relação de esquema $X$ onde só existe uma tupla, com o valor vazio $n_{A}$ em todos os seus atributos. Intuitivamente, $+\bowtie$ seleciona todos os valores dos atributos do primeiro operando, se um desses valores não ocorre no segundo operando, e acrescenta o valor vazio nos atributos que não são comuns aos dois operandos. O exemplo a seguir ilustra essas definições:

\begin{tabular}{|l|l|}
\multicolumn{1}{c}{$\mathrm{r}$} \\
\hline$A$ & $B$ \\
\hline \hline$a_{1}$ & $b_{1}$ \\
$a_{1}$ & $b_{2}$ \\
$a_{2}$ & $b_{4}$ \\
\hline
\end{tabular}

\begin{tabular}{|l|l|}
\multicolumn{1}{c}{$\mathrm{s}$} \\
\hline$B$ & $C$ \\
\hline \hline$b_{1}$ & $c_{1}$ \\
$b_{2}$ & $c_{2}$ \\
$b_{3}$ & $c_{3}$ \\
\hline
\end{tabular}

$r+\bowtie s$
\begin{tabular}{|l|l|l|}
\hline$A$ & $B$ & $C$ \\
\hline \hline$a_{1}$ & $b_{1}$ & $c_{1}$ \\
$a_{1}$ & $b_{2}$ & $c_{2}$ \\
$a_{2}$ & $b_{4}$ & $n_{A}$ \\
\hline
\end{tabular}

$r \bowtie_{+} s$
\begin{tabular}{|l|l|l|}
\hline$A$ & $B$ & $C$ \\
\hline \hline$a_{1}$ & $b_{1}$ & $c_{1}$ \\
$a_{1}$ & $b_{2}$ & $c_{2}$ \\
$n_{A}$ & $b_{3}$ & $c_{3}$ \\
\hline
\end{tabular}

\subsection{Propriedades de extensões da álgebra relaci- onal}

Um banco de dados com valores vazios pode ser entendido como representante de um conjunto de bancos de dados sem valores vazios, onde cada elemento neste conjunto 
pode ser obtido a partir de alguma substituição de valores vazios por elementos do domínio no banco inicial.

Esta idéia básica foi formalizada e trabalhada de diversas maneiras diferentes: Biskup [Bis83] chamou de "relações internas" as relações com valores vazios, que representam o conhecimento incompleto de um modelo real, representado por sua vez por relações sem valores vazios. Imielinski e Lipski [IL84] chamaram de $\operatorname{Rep}(r)$ o conjunto de relações (sem vazios) representados por uma relação $r$ com valores vazios. Liu e Sunderraman [LS90] chamaram de informação representada esse mesmo conjunto. Maier [Mai83] denotou $\operatorname{POSS}(r)$ o conjunto de relações sem vazios obtidos a partir de $r$ (com vazios), isto é, as possíveis relações representadas por $r$. A idéia de mundos possíveis, de Zimányi e Pirotte [ZP92] também corresponde ao mesmo conceito. Vamos usar aqui a terminologia $\operatorname{Rep}(r)$.

A generalização dos operadores relacionais para relações com valores vazios deve ser fiel à semântica definida pela função Rep. Para tal, os operadores devem possuir algumas propriedades básicas.

Em [IL84], temos a definição da propriedade "ideal" de tais extensões: o efeito de aplicar um operador $f$ sobre uma relação $r$ com valores vazios deveria ser o mesmo de aplicá-lo sobre todas as relações sem valores vazios em $\operatorname{Rep}(r)$, ou seja, $\operatorname{Rep}(f(r))=$ $f(\operatorname{Rep}(r))=\{f(r) \mid r \in \operatorname{Rep}(r)\}$. Como apontado em [IL84], esta propriedade é muito difícil de ser satisfeita. Por isso foram definidas propriedades mais fracas, que pudessem ser satisfeitas mais facilmente.

Em [Mai83], temos a definição da propriedade adequação de um operador. Um operador $f^{*}$ é uma extensão adequada do operador relacional $f$ se

$$
\operatorname{Rep}\left(f^{*}(r)\right) \supseteq f(\operatorname{Rep}(r))
$$

Intuitivamente, aplicando-se o operador $f^{*}$ à relação $r$ obtém-se uma nova relação $f^{*}(r)$, que representa um conjunto de relações sem valores vazios. Neste conjunto devem estar contidas todas as relações em $f(\operatorname{Rep}(r))$, ou seja, as relações obtidas aplicando o operador $f$ original às relações representadas pela relação original $r$.

Além disso, pode-se exigir que $f^{*}$ inclua o mínimo possível de informação adjcional, isto é, que não exista uma relação "intermediária" $r^{\prime}$ tal que $\operatorname{Rep}\left(f^{*}(r)\right) \supset$ $\operatorname{Rep}\left(r^{\prime}\right) \supseteq f(\operatorname{Rep}(r))$.

Em [ZP92] temos uma outra forma de abordar a questão: ao invés de se definir a semântica de um operador $f(r)$ através de seu efeito em todo o conjunto $\operatorname{Rep}(f(r))$, pode-se considerar apenas a informação que aparece em todas as relações em $\operatorname{Rep}(r)$. $\mathrm{O}$ operador estendido $f^{*}$ deve satisfazer então às propriedades: 
1. $\bigcap \operatorname{Rep}\left(f^{*}(r)\right)=\bigcap f(\operatorname{Rep}(r))$, ou seja, $f^{*}$ deve preservar as informações que aparecem em todas as representaçốes possíveis;

2. $f^{*}\left(g^{*}(r)\right)=f^{*} g^{*}(r)$, ou seja, os resultados intermediários no cálculo de uma subexpressão podem ser usados no cálculo da expressão completa.

A segunda condição evita problemas nas extensões dos operadores, decorrentes do fato de não considerar as iterações entre eles. Esta foi uma das motivações da definição de sistemas de representação em [IL84].

\subsection{Semântica de consultas com valores vazios}

Uma consulta a um banco de dados com valores vazios pode ser entendida de duas formas, dependendo da intenção de quem a formulou. Pode-se questionar o banco a respeito das informações concretas que ele possui ou a respeito das diversas possibilidades que ele representa. Por exemplo, considere o seguinte banco de dados:

\begin{tabular}{|l|l|l|}
\hline Nome & Cargo & Filial \\
\hline \hline Alice & Vendedor & Rio \\
Beto & Gerente & $n_{A}$ \\
\hline
\end{tabular}

onde o domínio do atributo Filial é $\{$ SPaulo, Rio, Vitoria $\}$. As seguintes relações representam as possibilidades:

\begin{tabular}{|l|l|l|}
\hline Nome & Cargo & Filial \\
\hline \hline Alice & Vendedor & Rio \\
Beto & Gerente & SPaulo \\
\hline
\end{tabular}

\begin{tabular}{|l|l|l|}
\hline Nome & Cargo & Filial \\
\hline \hline Alice & Vendedor & Rio \\
Beto & Gerente & Rio \\
\hline
\end{tabular}

\begin{tabular}{|l|l|l|}
\hline Nome & Cargo & Filial \\
\hline \hline Alice & Vendedor & Rio \\
Beto & Gerente & Vitoria \\
\hline
\end{tabular}

Considere também a consulta : "liste os nomes de funcionários da filial SPaulo".

Se interpretamos esta consulta da forma mais natural a resposta é "pode ser Beto". Se, por outro lado, a entendemos como "liste os possíveis funcionários da filial 
SPaulo" a resposta é "Beto". Se ainda, a interpretamos como "liste os funcionários que certamente são da filial SPaulo", a resposta é "não há".

A primeira interpretação corresponde à interpretação natural de um usuário que ignora se o banco de dados tem ou não valores vazios. Já a terceira pressupõe a não ocorrência de valores vazios, por exemplo. Para bancos de dados com informação incompleta, Lipski [Lip79] chamou estas interpretações de "externa" e "interna", respectivamente. Especificou também dois limites para a interpretação externa de uma consulta Q:

1. $\|Q\|_{*}$, o conjunto de objetos que se pode concluir, com certeza, que satisfazem as condições especificadas em Q;

2. $\|Q\|^{*}$, o conjunto de objetos que podem ou não satisfazer as condições especificadas em $Q$ (mas não se pode concluir que não satisfazem).

No exemplo:

- \| funcionários em SPaulo $\|_{*}=\Phi$

- $\|$ funcionários em SPaulo $\|^{*}=\{$ Beto $\}$

É interessante notar que o cálculo dos conjuntos $\|\mathrm{Q}\|_{*}$ e $\|\mathrm{Q}\|^{*}$ é relativamente simples de ser feito, quando se questiona sobre apenas um atributo. Quando a consulta envolve expressões booleanas mais complexas, o cálculo pode ficar bastante complicado, pois, em geral, não se pode decompor a expressão em subexpressões mais simples sem perdas. Por exemplo, nem sempre é verdade que $\left\|Q_{1} \vee Q_{2}\right\|_{*}=$ $\left\|Q_{1}\right\|_{*} \cup\left\|Q_{2}\right\|_{*}$. A seguinte situação ilustra um desses casos. Consideremos as relações Lidera(Líder) e Funcionários(Funcionário) a seguir.

\begin{tabular}{|c|c|}
\hline Lidera & Funcionários \\
\hline Lider & Funcionário \\
\hline $\begin{array}{l}\text { Alice } \\
n_{A}\end{array}$ & $\begin{array}{l}\text { Alice } \\
\text { Beto }\end{array}$ \\
\hline
\end{tabular}

Suponhamos que o domínio dos atributos Líder e Funcionários seja $\mathrm{D}=\{$ Alice, Beto\} e considere a consulta: \| Funcionários que são ou não líderes $\|_{*}$. A resposta é $\{$ Alice, Beto\}. Se, no entanto, decompomos a consulta, temos

- \| Funcionários que são líderes $\|_{*}=\{$ Alice $\}$ 
- $\|$ Funcionários que não são líderes $\|_{*}=\Phi$

Essa situação decorre do uso do valor vazio na relação Lidera. Nesse caso, não se pode afirmar que Beto é ou não um líder.

\subsection{Otimização de consultas com valores vazios}

A extensão do modelo relacional com vazios feita por Codd [Codd79] apresentava problemas na avaliação de consultas, decorrentes da utilização da lógica de 3 valores. Por exemplo, expressões do tipo (Idade $\geq 50) \vee($ Idade $\leq 50$ ) recebem o valor lógico $\mathbf{P}$ (possível) se a tupla no atributo Idade contém o valor vazio $n_{A}$. Este fato foi apontado pelo próprio Codd. Uma tupla nessas condições pode não aparecer no resultado final da consulta.

Muitos autores notaram essa característica e propuseram algoritmos para avaliação de consultas com expressões com valores vazios baseados em estudos de caso, isto é, análise do domínio do atributo e verificação de tautologias, como no exemplo anterior. Esta foi a proposta de Grant [Gra77]. Siklóssy [Sik81] propõe um algoritmo bastante eficiente para consultas simples, que descrevemos a seguir.

$\mathrm{O}$ algoritmo inicialmente transforma a consulta em uma expressão em lógica sentencial. O exemplo seguinte ilustra essa transformação:

\begin{tabular}{|l|l|l|l|l|}
\multicolumn{7}{|c}{ Funcionários } \\
\hline Cod & Nome & Depto & Idade & Est.Civil \\
\hline \hline C1 & Diana & Contabilidade & $n_{A}$ & Casado \\
C2 & Mario & $n_{A}$ & $n_{A}$ & Casado \\
\hline
\end{tabular}

Consultas sobre a relação Funcionários:

1. $($ Idade $\geq 50) \wedge($ Depto $=$ Contabilidade $) \vee$ $($ Idade $<50) \wedge($ Est. Civil $=$ Casado $)$

2. (Idade $\geq 50) \vee($ Idade $\leq 60)$

Sejam $\mathrm{P} 1=($ Idade $\geq 50), \mathrm{P} 2=($ Depto $=$ Contabilidade $), \mathrm{P} 3=($ Est.Civil $=$ Casado $)$ e $\mathrm{P} 4=($ Idade $\leq 60)$. As consultas transformadas são:

1. $\mathrm{P} 1 \wedge \mathrm{P} 2 \vee \neg \mathrm{P} 1 \wedge \mathrm{P} 3$ 


\section{2. $\mathrm{P} 1 \vee \mathrm{P} 4$}

As consultas tratadas por esse algoritmo são as formadas por combinações booleanas de termos primitivos na forma "nome do atributo op constante", onde op pode ser $=, \neq, \geq$, etc.

Cada termo primitivo é avaliado segundo a lógica de 3 valores, podendo assumir então os valores $\mathbf{V}, \mathbf{F}$ ou $\mathbf{P}$. Se o valor do termo é $\mathbf{V}$ ou $\mathbf{F}$, substitui-se o mesmo por seu valor na consulta transformada. Se o valor é $P$, não é feita nenhuma substituição. O resultado é uma expressão em cálculo proposicional, que pode ser calculada eficientemente, obtendo-se um dos valores V, F, P. Nesse caso, $\mathbf{P}$ indica que a expressão pode ser satisfeita.

No exemplo, o algoritmo faria as seguintes substituições:

1. $\mathrm{P} 1 \wedge \mathrm{P} 2 \vee \neg \mathrm{P} 1 \wedge \mathrm{P} 3$

na primeira tupla: $\mathrm{P} 1 \wedge \mathrm{V} \vee \neg \mathrm{P} 1 \wedge \mathrm{V}=\mathrm{P} 1 \vee \neg \mathrm{P} 1=\mathrm{V}$

na segunda tupla: $\mathrm{P} 1 \wedge \mathrm{P} 2 \vee \neg \mathrm{P} 1 \wedge \mathrm{V}=\mathrm{P} 1 \wedge \mathrm{P} 2 \vee \neg \mathrm{P} 1=\mathbf{P}$

2. $\mathrm{P} 1 \vee \mathrm{P} 4$

nas duas tuplas: $\mathrm{P} 1 \vee \mathrm{P} 4=\mathrm{P}$

O resultado da segunda consulta no exemplo poderia ser $\mathbf{V}$, pois a mesma representa uma tautologia para números inteiros. Para que o algoritmo a avalie corretamente, o banco de dados precisa incluir axiomas sobre os números inteiros.

Em linhas gerais, este é o algoritmo proposto em [Sik81]. O autor comenta que as consultas tratadas pelo algoritmo não são gerais, mas na prática são aceitáveis.

\subsection{Consultas em representação lógica}

Ao apresentar o formalismo lógico para a descrição de bancos de dados relacionais, Reiter [Rei84] também definiu uma notação especial para consultas a bancos de dados. Uma consulta em uma linguagem relacional $R=(A, W)$ é uma expressão da forma:

$$
\{\mathrm{x} / \mathrm{T} \mid \mathrm{W}(\mathrm{x})\}
$$

onde $\mathrm{x} / \mathrm{T}$ denota uma sequência $x_{1} / T_{1}, \ldots, x_{n} / T_{n}$, onde cada $x_{i}$ é uma variável distinta no alfabeto A e cada $T_{i}$ é um tipo simples em A. W(x) é uma fórmula cujas 
variáveis livres estão entre $x_{1}, \ldots, x_{n}$ e cujas variáveis quantificadas devem estar associadas a tipos. Se $n=0$ temos uma consulta tipo SIM/NÃO da forma $\{W(x)\}$, onde W não tem variáveis livres.

Intuitivamente, a resposta a uma consulta $\{x / T \mid W(x)\}$ é um conjunto de $n$ tuplas $\mathrm{x}=\left(x_{1}, \ldots, x_{n}\right)$ tal que cada $x_{i}$ é do tipo $T_{i}$ (ou seja, satisfaz o predicado $T_{i}$ ) e o banco de dados satisfaz a fórmula $W(x)$. A seguir temos alguns exemplos de consultas utilizando o seguinte banco de dados, representado na forma tabular:

\begin{tabular}{|l|}
\multicolumn{1}{l}{ Disciplinas } \\
\hline Disciplina \\
\hline \hline Geometria \\
Biologia \\
\hline
\end{tabular}

\begin{tabular}{|l|} 
Alunos \\
\hline Aluno \\
\hline \hline Alice \\
Beto \\
\hline
\end{tabular}

\begin{tabular}{|l|l|}
\multicolumn{2}{c}{ Matriculado } \\
\hline Aluno & Disciplina \\
\hline \hline Alice & Geometria \\
Beto & Biologia \\
Beto & Geometria \\
\hline
\end{tabular}

ou, de maneira equivalente, representado pelos axiomas:

- $\forall x \operatorname{Disciplina}(x) \Rightarrow(x=$ Geometria $) \vee(x=$ Biologia $)$

- $\forall x \operatorname{Aluno}(x) \Rightarrow(x=$ Geometria $) \vee(x=$ Biologia $)$

- $\forall x \forall y$ Matriculado $(x, y) \Rightarrow(x=$ Alice $\wedge y=$ Geometria $) \vee$

$(x=$ Beto $\wedge y=$ Biologia $) \vee$

( $x=$ Beto $\wedge y=$ Geometria)

As consultas podem então ser escritas na forma:

- Alunos matriculados em mais de uma disciplina:

$\{x /$ Aluno $\mid \exists y /$ Disciplina $\exists z /$ Disciplina $[\neg(y=z) \wedge \operatorname{Matriculado}(x, y) \wedge$ Matriculado $(x, z)]\}$

- Alunos matriculados em todas os disciplinas: $\{x /$ Aluno $\mid \forall y /$ Disciplina [Matriculado $(x, y)]\}$

- Pares de alunos matriculados na mesma disciplina: $\{x /$ Aluno, $y /$ Aluno $\mid \exists z /$ Disciplina [Matriculado $(x, z) \wedge \operatorname{Matriculado}(y, z)]\}$ 
Formalmente, uma n-tupla $\mathrm{c}=\left(c_{1}, \ldots, c_{n}\right)$ de constantes em A é uma resposta a uma consulta $\{\mathrm{x} / \mathrm{T} \mid \mathrm{W}(\mathrm{x})\}$ em relação ao banco de dados $\mathrm{BD}$ (onde $\mathrm{BD}$ é uma teoria relacional com valores vazios) se e somente se as fórmulas $T_{i}\left(c_{i}\right), \mathrm{i}=1, \ldots \mathrm{n}$ e $\mathrm{W}(\mathrm{c})$ podem ser provadas usando axiomas em $\mathrm{BD}$ e a lógica de primeira ordem com igualdade, isto é, se valem as condições:

1. $\mathrm{BD} \vdash T_{i}\left(c_{i}\right), \mathrm{i}=1,2, \ldots, \mathrm{n}$

2. $\mathrm{BD} \vdash \mathrm{W}(\mathrm{c})$

Note que, no caso de consultas tipo SIM/NÃO, o resultado pode ser um conjunto sem nenhuma tupla (denotado por \{\} ), significando a resposta NÃO, ou um conjunto com uma tupla vazia (denotado por $\{()\}$ ), significando a resposta SIM.

O conjunto de todas as respostas a uma dada consulta é denotado por $\| \mathrm{x} / \mathrm{T}$ | $\mathrm{W}(\mathrm{x}) \|_{B D}$. Quando for claro, pelo contexto, o banco de dados BD em uso pode-se escrever somente $\|\mathrm{x} / \mathrm{T} \mid \mathrm{W}(\mathrm{x})\|$.

\subsection{Algoritmos de consulta em representação lógica}

Utilizando uma representação lógica de bancos de dados (Cap.5), Reiter [Rei86] desenvolveu um algoritmo para avaliação de consultas em bancos de dados com valores vazios. Este algoritmo foi posteriormente estendido por Yuan e Chiang [YC88] para tratar informações disjuntivas. A seguir apresentamos o algoritmo proposto e sua extensão. A nomenclatura e notação utilizadas nessa seção são as mesmas do capítulo 5 .

\subsection{Algoritmo I}

O algoritmo consiste em decompor consultas seguidamente até alcançar subconsultas simples e facilmente calculáveis. Nesse processo de decomposição pode haver perda de informação e por este motivo o algoritmo pode não calcular todas as respostas a uma dada consulta. O primeiro tipo de decomposição é feito a partir dos resultados:

1. $\left\|\mathrm{x} / \mathrm{T}\left|W_{1}(\mathbf{x}) \wedge W_{2}(\mathbf{x})\|=\| \mathrm{x} / \mathrm{T}\right| W_{1}(\mathrm{x})\right\| \cap\left\|\mathrm{x} / \mathrm{T} \mid W_{2}(\mathbf{x})\right\|$

2. $\left\|\mathbf{x} / \mathbf{T}\left|W_{1}(\mathbf{x}) \vee W_{2}(\mathbf{x})\|\supseteq\| \mathbf{x} / \mathrm{T}\right| W_{1}(\mathrm{x})\right\| \cup\left\|\mathrm{x} / \mathrm{T} \mid W_{2}(\mathbf{x})\right\|$ 
A inclusão no sentido inverso (que levaria à igualdade) não é válida. O exemplo a seguir mostra uma dessas situações:

- $\mathrm{BD}=\{\forall x \mathrm{P}(x) \Rightarrow x=\mathrm{a}$, $\forall x \mathrm{~T}(x) \Rightarrow x=\mathrm{a} \vee x=\mathrm{b}\}$

ou, de forma equivalente, na notação tabular:

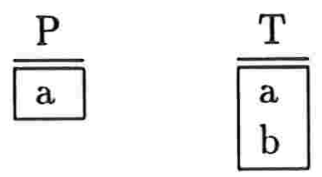

- $\|x / \mathrm{T} \mid \mathrm{P}(x) \vee \neg \mathrm{P}(x)\| \equiv$

Elementos do alfabeto que satisfazem o predicado $\mathrm{T}$ e a fórmula $\mathrm{P}(x) \vee \neg \mathrm{P}(x)$ $\equiv$ Elementos do alfabeto que satisfazem o predicado $\mathrm{T}$ (pois a fórmula indicada é uma tautologia $)=\{\mathrm{a}, \mathrm{b}\}$

- $\|x / \mathrm{T} \mid \mathrm{P}(x)\| \equiv$

Elementos que satisfazem os predicados $\mathrm{T}$ e $\mathrm{P}=\{\mathrm{a}\}$

- $\|x / \mathrm{T} \mid \neg \mathrm{P}(x)\| \equiv$

Elementos que satisfazem o predicado $\mathrm{T}$ e não satisfazem $\mathrm{P}=\{\}$

No caso, não é possível provar $\neg \mathrm{P}(\mathrm{b})$, pois não existe o axioma $\neg(\mathrm{a}=\mathrm{b})$

Notação: | T | é o conjunto de tuplas que aparece no axioma da extensão do predicado T. Se o axioma é da forma

$$
\begin{aligned}
\forall x_{1}, \ldots, \forall x_{m} \mathrm{~T}\left(x_{1}, \ldots, x_{m}\right) \Rightarrow \quad & x_{1}=c_{11} \wedge \ldots \wedge x_{m}=c_{1 m} \vee \\
& x_{1}=c_{21} \wedge \ldots \wedge x_{m}=c_{2 m} \vee \ldots \vee \\
& x_{1}=c_{k 1} \wedge \ldots \wedge x_{m}=c_{k m}
\end{aligned}
$$

então $|\mathrm{T}|=\left\{\left(c_{11}, \ldots, c_{1 m}\right), \ldots,\left(c_{k 1}, \ldots, c_{k m}\right)\right\}$

O próximo tipo de decomposição tem o objetivo de remover os quantificadores (universal e existencial). Para tanto, são utilizados os operadores relacionais de Divisão e Projeção.

Seja $\mathrm{S}$ um conjunto de $(\mathrm{n}+1)$-tuplas de constantes, BD uma teoria relacional e $\mathrm{T}$ um tipo simples. Então a divisão de $\mathrm{S}$ por $\mathrm{T}$ (em relação a $\mathrm{BD}$ ) é

$$
\mathrm{S} \div \mathrm{T}=\left\{\left(c_{1}, \ldots, c_{n}\right) \mid\left(c_{1}, \ldots, c_{n}, b\right) \in \mathrm{S}, \text { para todo } b \in|T|\right\}
$$


Esta definição só é válida quando existe pelo menos uma constante $b$ em $|\mathrm{T}|$.

Seja $\mathrm{S}$ um conjunto de $\mathrm{n}$-tuplas de constantes $(\mathrm{n} \geq 1), i_{1}, \ldots, i_{k}$ no intervalo $[1, \mathrm{n}]$. A projeção de $\mathrm{S}$ nos componentes $i_{1}, \ldots, i_{k}$ é

$$
\Pi_{i_{1}, \ldots, i_{k}}=\left\{\left(a_{1}, \ldots, a_{k}\right) \mid \exists\left(b_{1}, \ldots, b_{n}\right) \in \mathrm{S}, a_{j}=b_{i_{j}}, \mathrm{j}=1,2, \ldots \mathrm{k}\right\}
$$

A partir dessas definições podemos apresentar as decomposições que eliminam os quantificadores das consultas: - $\left\|\mathrm{x} / \mathbf{T}_{1} \mid \forall y / T_{2} W(\mathbf{x}, y)\right\|=\begin{aligned} \| & \mathbf{x} / \mathbf{T}_{1}, y / T_{2} \mid W(\mathrm{x}, y) \| \div T_{2}, \text { se }\left|T_{2}\right| \neq\{\}, \text { ou } \\ & T_{2} \mid, \text { caso contrario }\end{aligned}$

- $\left\|\mathbf{x} / \mathbf{T}_{1}\left|\exists y / T_{2} W(\mathbf{x}, y)\left\|\supseteq \Pi_{1,2, \ldots, n-1}\right\| \mathbf{x} / \mathbf{T}_{1}, y / T_{2}\right| W(\mathbf{x}, y)\right\|$ onde $\mathrm{x}=x_{1}, \ldots, x_{n}$

Assim como na decomposição de expressões envolvendo o conectivo $V$, as expressões que envolvem o quantificador $\exists$ também não podem ser decompostas sem perdas. Este fato é razoável, uma vez que o quantificador $\exists$ pode ser escrito como uma série de " $\mathrm{V}$ " encadeados (pois trabalhamos com um universo finito de constantes):

$$
\exists x W(x) \text { é equivalente a } W\left(a_{1}\right) \vee \ldots \vee W\left(a_{n}\right)
$$

onde $a_{1}, \ldots a_{n}$ são as constantes existentes.

O exemplo a seguir ilustra uma situação onde há perdas na decomposição:

- $\mathrm{BD}=\left\{\forall x D(x) \Rightarrow x=n_{A}\right.$ $\forall x C(x) \Rightarrow x=a \vee x=b \vee x=c$$$
\left.\forall x \forall y P(x, y) \Rightarrow\left(x=n_{A} \wedge y=a\right) \vee\left(x=n_{A} \wedge y=b\right)\right\}
$$

- Axioma de nome único: $\neg\left(n_{A}=c\right)$

O resultado da consulta

$$
\mathrm{Q}=\{x / D(x) \mid \exists y / C(y) P(x, y) \wedge \neg(y=c)\}
$$

é $n_{A}$, pois 


$$
\begin{array}{ll}
\mathrm{BD} \vdash & D\left(n_{A}\right) \wedge C(a) \wedge P\left(n_{A}, a\right) \wedge \neg\left(n_{A}=a\right) \vee \\
& D\left(n_{A}\right) \wedge C(b) \wedge P\left(n_{A}, b\right) \wedge \neg\left(n_{A}=b\right)
\end{array}
$$

pois as variáveis devem assumir um (e somente um) valor entre as constantes do alfabeto e, como sabemos que $n_{A} \neq c$, a fórmula $x \neq a \vee x \neq b$, quando $x$ assume o valor $n_{A}$, pode ser provada. Entretanto, não é possível provar separadamente:

$$
\begin{aligned}
& \mathrm{BD} \vdash D\left(n_{A}\right) \wedge C(a) \wedge P\left(n_{A}, a\right) \wedge \neg\left(n_{A}=a\right) \mathrm{e} \\
& \mathrm{BD} \vdash D\left(n_{A}\right) \wedge C(b) \wedge P\left(n_{A}, b\right) \wedge \neg\left(n_{A}=b\right)
\end{aligned}
$$

Por outro lado o algoritmo vai transformar a consulta em

$$
\mathrm{Q}=\{x / D(x) y / C(y) \mid P(x, y) \wedge \neg(y=c)\}
$$

cujo resultado é \{\} , ou seja, não vai encontrar o elemento $n_{A}$ que era a resposta da consulta original. Por este motivo o algoritmo devolve um conjunto de respostas (no caso, vazio) que está contido no conjunto de respostas correto (no caso, $\left\{n_{A}\right\}$ ). Este é um dos motivos que tornam o algoritmo incompleto.

A decomposição a seguir é usada em consultas onde é especificada, para a resposta, uma variável que não ocorre livre na fórmula, isto é, uma consulta do tipo $\| \mathrm{x} / T_{1}$, $y / T_{2}, \mathbf{z} / T_{3} \mid \mathrm{W}(\mathbf{x}, \mathbf{z}) \|$. Nesse caso, a variável $y$ não influencia no cálculo da consulta, mas a resposta deve conter todas as constantes do tipo $T_{2}$, na posição especificada. Assim, temos:

$$
\begin{gathered}
\left\|\mathrm{x} / T_{1}, \mathrm{y} / T_{2}, \mathrm{z} / T_{3} \mid \mathrm{W}(\mathrm{x}, \mathrm{z})\right\|= \\
\prod_{2, \ldots, n+1,1, n+2, \ldots, n+k}\left(\left|T_{2}\right| \times\left\|\mathrm{x} / T_{1}, \mathrm{z} / T_{3} \mid \mathrm{W}(\mathrm{x}, \mathrm{z})\right\|\right)
\end{gathered}
$$

onde $\mathbf{x}=\left(x_{1}, \ldots, x_{n}\right), \mathbf{z}=\left(z_{1}, \ldots, z_{k}\right), \mathrm{e} \times$ denota o produto cartesiano entre tuplas.

Após aplicar adequada e repetidamente as decomposições descritas, o resultado é uma série de sub-consultas "básicas" dos tipos $\|\mathrm{x} / \mathrm{T}|\mathrm{P}(\mathrm{r})\|\mathrm{e}\| \mathrm{x} / \mathrm{T}| \neg \mathrm{P}(\mathrm{r})\|$. Para detalhar o cálculo dessas sub-expressões definimos alguns conceitos auxiliares.

Seja $\mathbf{r}$ uma n-tupla $\left(r_{1}, \ldots, r_{n}\right)$ de variáveis ou constantes, e $\mathrm{S}$ um conjunto de $\mathrm{n}$-tuplas de constantes da forma $\left(t_{1}, \ldots, t_{n}\right)$. Intuitivamente, $\mathrm{S}$ pode ser entendida como uma relação (um conjunto de tuplas). Então

$$
\begin{aligned}
\Sigma_{\mathbf{r}}(\mathrm{S})=\{\mathrm{t} \in \mathrm{S} \mid & \text { para } \mathrm{i}=1, \ldots \mathrm{n}, \text { se } r_{i} \text { é constante, então } \\
& t_{i}=r_{i}, \text { e se } r_{i} \text { é uma variável } x, \mathrm{e} \\
& r_{i_{1}}, \ldots, r_{i_{k}} \text { são os componentes de } \mathrm{r} \\
& \text { tais que } r_{i_{1}}=\ldots=r_{i_{k}}=x, \text { então } \\
& \left.t_{i_{1}}=\ldots=t_{i_{k}}\right\}
\end{aligned}
$$


$\Sigma_{\mathbf{r}}$ corresponde, de certa forma, ao operador de seleção. Por exemplo, se consideramos $\mathrm{r}=(x, y, a, x, z, y)$, e um conjunto $\mathrm{S}$ de tuplas do tipo $\left(t_{1}, \ldots, t_{6}\right)$, ou seja, vemos $\mathrm{S}$ como uma relação em um esquema $R=\left\{A_{1}, A_{2}, A_{3}, A_{4}, A_{5}, A_{6}\right\}$, então $\Sigma_{x, y, a, x, z, y}$ pode ser entendido como $\sigma\left(A_{1}=A_{4} \wedge A_{2}=A_{6} \wedge A_{3}=a\right)$. Dessa forma, todas as restrições descritas pelo conjunto $\mathrm{r}$ são satisfeitas. Note que a variável $z$ não induz nenhuma restrição.

O cálculo de uma consulta básica (sem negação) é feito da forma:

$$
\left\|\mathrm{x} / \mathrm{T}|\mathrm{P}(\mathrm{r}) \|=| \mathrm{T} \mid \cap \prod_{i_{1}, \ldots, i_{n}} \Sigma_{\mathbf{r}}(|P|)\right.
$$

onde $\mathbf{x}=x_{1}, \ldots, x_{n}, \mathbf{r}=r_{1}, \ldots, r_{m}, r_{i}$ pode ser constante ou variável, e $r_{i j}$ é a primeira ocorrência de $r$.

Intuitivamente, estamos retirando do predicado $\mathrm{P}$ as tuplas desejadas e reposicionando suas colunas para que obedeçam à ordenação solicitada.

No caso especial onde o predicado $\mathrm{P}$ é a igualdade, o cálculo fica mais fácil:

- $\|a=b\|=\left\{\begin{array}{l}\{()\}(\mathrm{SIM}) \text { se } a \text { e } b \text { são constantes idênticas } \\ \{\}(\mathrm{NA} O) \text { se } a \text { e } b \text { são constantes diferentes }\end{array}\right.$

- $\|x / T|x=x \|=| T \mid$

- $\|x / T|x=a\|=\| x / T| a=x\|=\left\{\begin{array}{l}\{(a)\} \text { se } a \in|\mathrm{T}| \\ \{\} \text { se } a \notin|\mathrm{T}|\end{array}\right.$

- $\left\|x / T_{1}, y / T_{2} \mid x=y\right\|=\left\{(c, c)|c \in| T_{1} \mid\right.$ e $\left.c \in\left|T_{2}\right|\right\}$

Para o cálculo de expressões do tipo $\|\mathrm{x} / \mathrm{T} \mid \neg \mathrm{P}(\mathrm{r})\|$ são necessárias mais definições auxiliares:

- Seja $\mathbf{r}=r_{1}, \ldots, r_{n}, \mathbf{x}=x_{1}, \ldots, x_{m}, \mathrm{e} \mathbf{c}=c_{1}, \ldots, c_{m}$, onde cada $r_{i}$ pode ser variável ou constante, $x_{i}$ são variáveis e $c_{i}$ são constantes. A substituição de $\mathrm{x}$ por $\mathrm{c}$ em $\mathbf{r}$, denotada por $\mathbf{r}_{\mathbf{c} \mid \mathbf{x}}$ é a $\mathbf{n}$-tupla obtida a partir de $\mathbf{r}$ substituindo cada ocorrência de $x_{i}$ por $c_{i}$, para $\mathrm{i}=1,2 \ldots, \mathrm{n}$. Por exemplo, $(x, y, a, x, z, y)_{(b, c, d) \mid(y, x, z)}$ $=(\mathrm{c}, \mathrm{b}, \mathrm{a}, \mathrm{c}, \mathrm{d}, \mathrm{b})$.

- Seja BD uma teoria relacional com valores vazios. Duas tuplas de constantes a e b discordam na teoria $\mathrm{BD}$ se e somente se $\mathrm{BD} \vdash \neg(\mathrm{a}=\mathrm{b})$.

- Seja S um conjunto de m-tuplas de constantes; $\mathrm{x}$ uma tupla com $\mathrm{m}$ variáveis distintas; $r$ uma $n$-tupla $(n \geq m)$ de constantes ou variáveis, onde as variáveis de $\mathbf{r}$ são as mesmas de $\mathbf{x}$; $\mathrm{T}$ um conjunto de $\mathrm{n}$-tuplas de constantes. Definimos o operador $D_{x, r}(\mathrm{~S}, \mathrm{~T})=\left\{\mathrm{c} \in \mathrm{S} \mid \mathrm{r}_{\mathbf{c} \mid \mathbf{x}}\right.$ discorda de toda tupla $\left.\mathrm{t} \in \mathrm{T}\right\}$. 
O cálculo de consultas da forma $\|\mathbf{x} / \mathbf{T} \mid \neg \mathrm{P}(\mathbf{r})\|$, onde $\mathrm{P}$ não é o predicado de igualdade, fica:

$$
\|\mathbf{x} / \mathbf{T} \mid \neg \mathrm{P}(\mathbf{r})\|=D_{\mathbf{x}, \mathbf{r}}(|T|,|P|)
$$

Para o caso especial onde $\mathrm{P}$ é a igualdade, temos : (onde $\mathrm{U}$ é o conjunto de axiomas de nome único da teoria em questão, e $a, b$ e $c$ são constantes)

- $\|\neg(\mathrm{a}=\mathrm{b})\|=\left\{\begin{array}{l}\{()\}(\mathrm{SIM}), \text { se } \neg(a=b) \text { ou } \neg(b=a) \in \mathrm{U} \\ \{\}(\mathrm{NA} \mathrm{O}), \text { caso contrário }\end{array}\right.$

- $\|x / T \mid \neg(x=x)\|=\{\}(\mathrm{N} \tilde{\mathrm{O}})$

- $\|x / T|\neg(x=a)\|=\| x / T| \neg(a=x)\|$ $=\{(c)|c \in| T \mid, \mathrm{e} \neg(c=a) \in \mathrm{U}$ ou $\neg(a=c) \in \mathrm{U}\}$

- $\left\|\mathrm{x} / T_{1}, \mathrm{y} / T_{2} \mid \neg(x=y)\right\|=\left\{(\mathrm{a}, \mathrm{b})|\mathrm{a} \in| T_{1}|, \mathrm{~b} \in| T_{2} \mid \mathrm{e}\right.$ $\neg(\mathrm{a}=\mathrm{b}) \in \mathrm{U}$ ou $\neg(\mathrm{b}=\mathrm{a}) \in \mathrm{U}\}$

Só resta agora especificar como calcular $D_{x, r}(\mathrm{~S}, \mathrm{~T})$, isto é, como determinar se duas tuplas a e b discordam. De modo equivalente, pode-se determinar quando a e b não podem ser iguais, ou seja, em que casos, ao igualarmos $a_{1}, \ldots, a_{n}$ e $b_{1}, \ldots, b_{n}$ obtemos uma teoria inconsistente. Isso pode ser feito da seguinte forma:

- Determinar as classes de equivalência sob o operador de igualdade, no conjunto $\left\{a_{1}, \ldots, a_{n}, b_{1}, \ldots, b_{n}\right\}$

- Se alguma das classes obtidas contém um par (c,c') e existe um axioma de nome único $\neg\left(c=c^{\prime}\right)$, então a teoria obtida é inconsistente, e portanto a discorda de b

O algoritmo descrito calcula corretamente a resposta de uma dada consulta, no sentido de que não fornece tuplas que não satisfazem as condições especificadas. Entretanto, em alguns casos a decomposição "perde" tuplas, como nos casos das decomposições envolvendo o conectivo $\vee$ ou o quantificador $\exists$. Reiter mostrou, no entanto, que em alguns casos o algoritmo é também completo, fornecendo todas as tuplas que satisfazem as condições. São eles:

- consultas que só envolvem o quantificador universal $\forall$ e o conectivo $\wedge$ (pois neste caso as decomposições não têm perdas) 
- consultas positivas (não aparece o conectivo $\neg$ ) (também não há perdas)

- consultas a bancos de dados sem valores vazios (neste caso temos todos os axiomas de nomes únicos presentes)

Para exemplificar o uso do algoritmo, vamos calcular a primeira consulta descrita nos exemplos de consultas da seção anterior.

Alunos matriculados em mais de uma disciplina: $\| x /$ Aluno $\mid \exists y /$ Disciplina $\exists z /$ Disciplina $[\neg(y=z) \wedge \operatorname{Matriculado}(x, y) \wedge \operatorname{Matriculado}(x, z)] \|=$

$\Pi_{1}(\| x /$ Aluno, $y /$ Disciplina $\mid \exists z /$ Disciplina $[\neg(y=z) \wedge \operatorname{Matriculado}(x, y) \wedge$ Matriculado $(x, z)] \|)=$

$\Pi_{1} \Pi_{1}(\| x /$ Aluno, $y /$ Disciplina, $z /$ Disciplina $\mid[\neg(y=z) \wedge$ Matriculado $(x, y) \wedge$ Matriculado $(x, z)] \|)=$

A expressão entre parênteses, quando decomposta pela regra do conectivo " $\wedge$ ", origina as três subconsultas a seguir:

1. $\| x /$ Aluno, $y /$ Disciplina, $z /$ Disciplina $\mid \neg(y=z) \| \cap$

2. $\| x /$ Aluno, $y /$ Disciplina, $z /$ Disciplina $\mid \operatorname{Matriculado}(x, y) \| \cap$

3. $\| x /$ Aluno, $y /$ Disciplina, $z /$ Disciplina $\mid \operatorname{Matriculado}(x, z) \|$

Calculando separadamente as subconsultas:

Primeira:

|| $x$ /Aluno, $y /$ Disciplina, $z /$ Disciplina $\mid \neg(y=z) \|=$ | Aluno | $\times \| y /$ Disciplina, $z /$ Disciplina $\mid \neg(y=z) \|=$ $\{$ Alice, Beto $\} \times\{($ Geometria, Biologia $),($ Biologia, Geometria $)\}=$ \{(Alice, Geometria, Biologia), (Alice, Biologia, Geometria), (Beto, Geometria, Biologia), (Beto, Biologia, Geometria)\}

Segunda:

$\| x /$ Aluno, $y /$ Disciplina $z /$ Disciplina $\mid$ Matriculado $(x, y) \|=$ $\Pi_{2,3,1}(\mid$ Disciplina $\mid \times \| x /$ Aluno, $y /$ Disciplina $\mid$ Matriculado $(x, y) \|=$ $\Pi_{2,3,1}(\{$ Geometria, Biologia $\} \times\{($ Alice, Geometria $),($ Beto, Biologia $)$, $($ Beto, Geometria $)\})=$ $\Pi_{2,3,1}(\{($ Geometria, Alice, Geometria), (Geometria, Beto, Biologia), (Geometria, Beto, Geometria), (Biologia, Alice, Geometria), (Biologia, Beto, Biologia), (Biologia, Beto, Geometria) $\})=$ 
$\{($ Alice, Geometria, Geometria), (Beto, Biologia, Geometria), (Beto, Geometria, Geometria), (Alice, Geometria, Biologia), (Beto, Biologia, Biologia), (Beto, Geometria, Biologia)\}

Terceira:

$\| x /$ Aluno, $y /$ Disciplina $z /$ Disciplina $\mid$ Matriculado $(x, z) \|=$

$\Pi_{2,1,3}(\mid$ Disciplina $\mid \times \| x /$ Aluno, $z /$ Disciplina $\mid \operatorname{Matriculado}(x, z) \|=$

$\Pi_{2,1,3}(\{$ Geometria, Biologia $\} \times\{($ Alice, Geometria $),($ Beto, Biologia $)$,

(Beto, Geometria) $\})=$

$\Pi_{2,1,3}$ (\{(Geometria, Alice, Geometria), (Geometria, Beto, Biologia), (Geometria, Beto, Geometria), (Biologia, Alice, Geometria), (Biologia, Beto, Biologia), (Biologia, Beto, Geometria) $\})=$

(\{(Alice, Geometria, Geometria), (Beto, Geometria, Biologia), (Beto, Geometria, Geometria), (Alice, Biologia, Geometria), (Beto, Biologia, Biologia), (Beto, Biologia, Geometria)\})

A intersecção das três subconsultas resulta em:

\{(Beto, Biologia, Geometria), (Beto, Geometria, Biologia)\}

Portanto, a resposta final à consulta, calculadas as duas projeções sobre o primeiro elemento das tuplas é $\{$ Beto\}. Este é um dos exemplos onde o algoritmo calcula todas as respostas a uma dada consulta.

\subsection{Algoritmo II}

O algoritmo da seção 6.7 é incompleto, isto é, não necessariamente calcula todas as respostas possíveis para uma dada consulta. Yuan e Chiang [YC88] notaram que um dos motivos dessa incompletude é ignorar informação indefinida (disjunções) em bancos de dados relacionais, como ilustrado no exemplo a seguir.

Seja $R=\{A, B\}$ um esquema de banco de dados relacional, $\operatorname{Dom}(A)=\{\alpha\}$, $\operatorname{Dom}(B)=\left\{a, b, n_{A}\right\}$. Sabe-se que $a \neq b$, mas $n_{A}$ pode ser igual a $a$ ou $b$. Seja $r(R)=\{(\alpha, a),(\alpha, b)\}$ e a consulta $\mathrm{Q}=\{\mathrm{x} \in \operatorname{Dom}(A) \mid \exists \mathrm{y} \in \operatorname{Dom}(B)[(\mathrm{x}, \mathrm{y}) \in r \wedge \mathrm{y}$ $\left.\left.\neq n_{A}\right]\right\}$. A resposta à questão $\mathrm{Q}$ (notação $\|\mathrm{Q}\|$ ) é $\|\mathrm{Q}\|=\{\alpha\}$, pois $a \neq n_{A} \vee b \neq n_{A}$.

Yuan e Chiang estenderam o conceito de resposta a uma consulta e modificaram o algoritmo de Reiter para devolver respostas definidas e indefinidas. Assim, conseguiram um algoritmo completo, mas perderam em complexidade, pois o algoritmo ficou computacionalmente mais complexo. A seguir apresentamos as idéias básicas em [YC88] e seus principais resultados.

Seja $R=(A, W)$ uma linguagem relacional, $B D \subseteq W$ uma teoria relacional que 
permite o uso de valores nulos, $\mathrm{Q}=\{\mathrm{x} / \mathrm{T} \mid \mathrm{W}(\mathrm{x})\}$ uma consulta a $\mathrm{BD}, \mathrm{t}=\left\{t_{1}, \ldots, t_{m}\right\}$ onde $\mathrm{m} \geq 1, t_{i}=\left(c_{i_{1}}, \ldots, c_{i_{n}}\right)$ uma n-tupla de constantes em A. $\mathrm{t}$ é uma resposta à consulta $\mathrm{Q}$ em relação a $\mathrm{BD}$ se e somente se:

1. $\mathrm{BD} \vdash T_{i}\left(c_{j_{\mathrm{i}}}\right), \mathrm{i}=1, \ldots, \mathrm{n}, \mathrm{j}=1, \ldots, \mathrm{m}$

2. $\mathrm{BD} \vdash \mathrm{W}\left(t_{1}\right) \vee \ldots \vee \mathrm{W}\left(t_{m}\right)$

3. $\mathrm{BD} \forall \mathrm{W}\left(t_{1}\right) \vee \ldots \vee \mathrm{W}\left(t_{i-1}\right) \vee \mathrm{W}\left(t_{i+1}\right) \vee \ldots \vee \mathrm{W}\left(t_{m}\right), \mathrm{i}=1,2, \ldots, \mathrm{m}$

A terceira condição garante que, a partir dos fatos no banco de dados, só conseguimos provar a disjunção, pois se retiramos qualquer um dos elementos da mesma, já não se consegue provar a fórmula.

Dessa forma, permitimos que a resposta a uma consulta seja indefinida. Quando $\mathrm{m}=1$, essa definição é equivalente à definição anterior de resposta. A seguinte notação é usada para distinguir respostas definidas/indefinidas:

- $\|\mathrm{Q}\|_{D}=\{\mathbf{t} \in\|\mathrm{Q}\||| \mathbf{t} \mid=1\}$

- $\|\mathrm{Q}\|_{I}=\{\mathrm{t} \in\|\mathrm{Q}\||| \mathrm{t} \mid>1\}$

Uma relação estendida $r(R)$ é uma relação que permite a representação de tuplas indefinidas (disjunções), isto é:

$$
r_{x}=\left\{t_{x}=\left\{t_{1}, \ldots, t_{m}\right\}, t_{i} \in \operatorname{Dom}\left(A_{1}\right) \times \ldots \times \operatorname{Dom}\left(A_{n}\right)\right\},
$$

onde $A_{i}$ são os atributos em $\mathrm{R}$. Quando $\mathrm{m}=1$, temos a definição usual de relação.

Os operadores relacionais são estendidos da seguinte forma (onde $r_{x 1}$ e $r_{x 2}$ são relações estendidas) :

- $r_{x 1} \cup r_{x 2}=\left\{t_{x} \mid t_{x} \in r_{x 1}\right.$ ou $\left.t_{x} \in r_{x 2}\right\}$

- $r_{x 1} \cap r_{x 2}=\left\{t_{x} \mid t_{x} \in r_{x 1}\right.$ e para cada $\mathrm{t} \in t_{x}, \mathrm{t} \in t_{x 2}$, para algum $t_{x 2} \in r_{x 2}$, ou $t_{x} \in r_{x 2}$ e para cada $\mathrm{t} \in t_{x}, \mathrm{t} \in t_{x 1}$, para algum $\left.t_{x 1} \in r_{x 1}\right\}$

- $\sigma_{F}\left(r_{x 1}\right)=\left\{t_{x} \mid t_{x} \in r_{x 1}\right.$ e $\mathrm{F}(t)$ é verdadeiro, para todo $\left.t \in t_{x}\right\}$

- $\Pi_{X}\left(r_{x 1}\right)=\left\{t_{x} \mid \exists s \in r_{x 1}\right.$ tal que $\left.t_{x}=\Pi_{X}(s)\right\}$

- $r_{x 1} \times r_{x 2}=\left\{t_{x} \mid \exists t_{x 1} \in r_{x 1}, \exists t_{x 2} \in r_{x 2}\right.$ tal que $\left.t_{x}=t_{x 1} \times t_{x 2}\right\}$ 
- $r_{x 1} \div r=\left\{t_{x} \mid t_{x}=\left\{t_{1}, \ldots, t_{n}\right\}\right.$ e para todo $\left.t \in r,\left\{t_{1} t, \ldots, t_{n} t\right\} \in r_{x 1}\right\}$

(Observação : a divisão só é definida quando o dividendo é uma relação estendida e o divisor é uma relação usual)

O algoritmo proposto também consiste em decompor as consultas. Essas decomposições precisam ser re-estudadas, pois agora as respostas às consultas podem ser indefinidas ou definidas ( $\|\mathrm{Q}\|_{D}$ ou $\|\mathrm{Q}\|_{I}$ ). Muitas dessas decomposições são análogas às anteriores. Outras são os resultados que garantem a completude do algoritmo:

- $\left\|\mathbf{x} / \mathbf{T}\left|W_{1}(\mathbf{x}) \wedge W_{2}(\mathbf{x})\left\|_{D}=\right\| \mathbf{x} / \mathbf{T}\right| W_{1}(\mathbf{x})\right\|_{D} \cap\left\|\mathbf{x} / \mathbf{T} \mid W_{2}(\mathbf{x})\right\|_{D}$

- $\left\|\mathbf{x} / \mathbf{T}\left|W_{1}(\mathbf{x}) \vee W_{2}(\mathbf{x})\left\|_{I}=\right\| \mathbf{x} / \mathbf{T}\right| W_{1}(\mathbf{x})\right\|_{I} \cup\left\|\mathbf{x} / \mathbf{T} \mid W_{2}(\mathbf{x})\right\|_{I}$

- $\left\|\mathbf{x} / \mathbf{T}_{1} \mid \exists \mathbf{y} / \mathbf{T}_{\mathbf{2}} W(\mathbf{x}, \mathbf{y})\right\|=\Pi_{x}\left(\|\left(\mathbf{x} / \mathbf{T}_{1}, \mathbf{y} / \mathbf{T}_{\mathbf{2}} \mid W(\mathbf{x}, \mathbf{y}) \|\right)\right.$

No caso particular onde $W_{1}$ e $W_{2}$ são consultas positivas (isto é, não envolvem o conectivo $\neg$ ), temos ainda:

- $\left\|\mathbf{x} / \mathbf{T}\left|W_{1}(\mathbf{x}) \wedge W_{2}(\mathbf{x})\|=\| \mathbf{x} / \mathbf{T}\right| W_{1}(\mathbf{x})\right\| \cap\left\|\mathbf{x} / \mathbf{T} \mid W_{2}(\mathbf{x})\right\|$

- $\left\|\mathbf{x} / \mathbf{T}\left|W_{1}(\mathbf{x}) \vee W_{2}(\mathbf{x})\left\|_{D}=\right\| \mathbf{x} / \mathbf{T}\right| W_{1}(\mathbf{x})\right\|_{D} \cup\left\|\mathbf{x} / \mathbf{T} \mid W_{2}(\mathbf{x})\right\|_{D}$

A idéia do algoritmo correto e completo é usar os resultados acima para as decomposições e computar separadamente as componentes $\|\mathrm{Q}\|_{D}$ e $\|\mathrm{Q}\|_{I}$ de uma dada consulta $\|\mathrm{Q}\|$. Na verdade, $Q$ é transformada em uma consulta $Q$ ' em um formato chamado "pseudo forma normal", e o trabalho é feito sobre esta consulta.

O custo computacional desse algoritmo é bem maior. Esse é o preço para se obter a completude. É interessante notar que nos casos em que o algoritmo já era completo anteriormente, a complexidade computacional não aumenta . 


\section{Capítulo 7}

\section{Atualização}

A atualização de bancos de dados relacionais, mesmo sem considerar o problema dos valores nulos, tem sido objeto de estudo de vários autores, principalmente porque requer uma série de verificações para se manter a integridade física e lógica do banco de dados. Casanova e Bernstein [CB80], por exemplo, apresentam um estudo desse problema através da lógica.

Em bancos de dados com valores nulos, devem ser considerados alguns aspectos adicionais em relação à atualização :

- qual a semântica das operações de atualização (inserção, alteração, remoção)?

- existem outras operações de atualização definidas, além das usuais?

- os problemas de atualização em bancos de dados sem valores nulos têm correspondentes em bancos com valores nulos?

A atualização em bancos de dados está fortemente relacionada com a verificação de restrições de integridade. Por isso, iniciamos a discussão sobre atualização discorrendo sobre restrições de integridade em bancos de dados com valores nulos. Em seguida apresentamos alguns resultados na área de atualização propriamente dita.

\subsection{Restrições de Integridade}

Restrições de integridade são sentenças lógicas que traduzem propriedades que o banco de dados deve satisfazer. Dizemos que uma relação $r$ satisfaz uma restrição $\delta$ se os modelos de $r$ são modelos de $\delta$, no sentido da lógica matemática. Uma relação 
$r$ satisfaz um conjunto de restrições $\Sigma$ se $r$ satisfaz cada $\delta \in \Sigma$. Dado um conjunto de restrições $\Sigma$ definidos sobre um esquema R, $\operatorname{Sat}(\Sigma)$ é o conjunto de relações em R que satisfazem $\Sigma$.

As dependências são um tipo muito importante de restrições, que englobam as dependências funcionais, multivaloradas ou de junção. Nesta seção apresentamos a noção de completamento, e seu significado para bancos de dados com valores nulos. Estes conceitos são apresentados como em [ZP92].

O completamento de uma relação $r$ com respeito a um conjunto de dependências $\Sigma$, denotado por $\operatorname{comp}_{\Sigma}(r)$ é uma relação $s$ que satisfaz:

- $r \subseteq s$

- $s \subseteq \operatorname{Sat}(\Sigma)$

- para toda $s^{\prime}$ que satisfaz os itens anteriores, $s \subseteq s^{\prime}$

Intuitivamente $s$ é uma relação obtida de $r$ onde foram incluídas as tuplas necessárias para que as dependências em $\Sigma$ sejam satisfeitas. Em particular, se $r$ já satisfaz as dependências em $\Sigma, s=r$. A primeira condição garante que a informação em $r$ não é perdida; a segunda garante que o completamento satisfaz as dependências em $\Sigma$, e a terceira condição garante a minimalidade do completamento, no sentido em que se inclua o mínimo possível de tuplas. O completamento, se existir, é único, e se não existir, é porque o banco original era inconsistente.

No caso de bancos de dados com valores nulos, para definir o conceito de completamento deve-se considerar as diversas possibilidades que o banco representa. Considere o exemplo a seguir [ZP92], onde temos algumas das possibilidades em $\operatorname{Rep}(r)$, para uma relação hipotética $r$ :

\begin{tabular}{|l|l|l|}
\hline Professor & Discip & Depto \\
\hline \hline Paulo & Cálculo & Zoologia \\
Paulo & Álgebra & Botânica \\
\hline
\end{tabular}

\begin{tabular}{|l|l|l|}
\hline Professor & Discip & Depto \\
\hline \hline Tomas & Cálculo & Zoologia \\
Ana & Cálculo & Botânica \\
\hline
\end{tabular}

\begin{tabular}{|l|l|l|}
\hline Professor & Discip & Depto \\
\hline \hline Ana & Cálculo & Zoologia \\
Ana & Cálculo & Botânica \\
Ana & Álgebra & Zoologia \\
Ana & Álgebra & Botânica \\
\hline
\end{tabular}


Seja também o conjunto de dependências $\Sigma=\left\{\delta_{1}=\right.$ Professor $\rightarrow$ Discip, $\delta_{2}=$ Discip $\rightarrow$ Professor $\}$, onde $\rightarrow \mathrm{e} \rightarrow$ representam, respectivamente, dependências multivaloradas e dependências funcionais (Cap.2). Como cada relação em $\operatorname{Rep}(r)$ não possui valores nulos, pode-se generalizar trivialmente a noção de satisfabilidade de dependências exigindo $\operatorname{Rep}(r) \subseteq \operatorname{Sat}(\Sigma)$. Para satisfazer $\delta_{1}$, as tuplas (Paulo, Cálculo, Botânica) e (Paulo, Álgebra, Zoologia) devem ser adicionadas à primeira relação em $\operatorname{Rep}(r)$. A segunda relação não satisfaz $\delta_{2}$, e deve então ser retirada do completamento. A terceira relação já satisfaz $\delta_{1}$ e $\delta_{2}$.

Assim, o completamento de $\operatorname{Rep}(r)$ em relação a $\Sigma$ é obtido tomando o completamento de cada relação em $\operatorname{Rep}(r)$, se existir:

$$
\operatorname{Comp}_{\Sigma}(\operatorname{Rep}(r))=\left\{\operatorname{comp}_{\Sigma}(s) \mid s \in \operatorname{Rep}(r)\right\}
$$

Do ponto de vista sintático, é necessário definir uma função $\overline{C o m p}$ para transformar $r$ de forma que sua representação $\operatorname{Rep}(r)$ satisfaça as dependências em $\Sigma$, ou seja, $\operatorname{Rep}(r) \subseteq \operatorname{Sat}(\Sigma)$. Em outras palavras, são retiradas de $\operatorname{Rep}(r)$ as possibilidades que não satisfazem $\Sigma$, considerando apenas $\operatorname{Rep}(r) \cap \operatorname{Sat}(\Sigma)$. Além disso, $\overline{\operatorname{Comp}}$ deve ser definida de modo que as relaçôes obtidas sejam as mesmas que seriam obtidas se completássemos cada relação separadamente, como indica a figura a seguir:

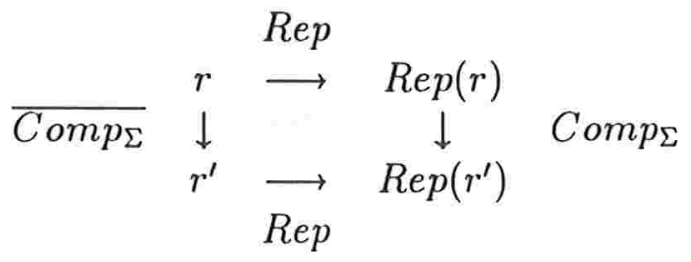

Para definir tal função com valores vazios, é necessário usar estruturas mais complexas que relações com valores nulos. Por exemplo, considere a seguinte relação [ZP92]:

\begin{tabular}{|l|l|l|}
\multicolumn{3}{c}{ Leciona } \\
\hline Professor & Discip & Depto \\
\hline \hline Ana & Cálculo & Zoologia \\
$n_{A}$ & Álgebra & Botânica \\
\hline
\end{tabular}

e a dependência $\delta_{1}=$ Professor $\rightarrow$ Discip. Para representar $\overline{C o m p_{\delta_{1}}}$ (Leciona) é preciso expressar o fato que $n_{A} \neq$ Ana. Considere agora a mesma relação e a dependência multivalorada $\delta_{2}=$ Professor $\rightarrow$ Discip. Uma relação em $C o m p_{\delta_{2}}(R e p($ Leciona $))$ deve conter duas ou quatro tuplas, dependendo do valor de $n_{A}$. Portanto, para definir $\overline{\mathrm{Comp}_{\delta_{2}}}$ é necessária uma C-tabela (Cap.4) do tipo: 


\begin{tabular}{|c|c|c|c|}
\hline Professor & Discip & Depto & Condição \\
\hline Ana & Cálculo & Zoologia & \\
$n_{A}$ & Álgebra & Botânica & \\
$n_{A}$ & Cálculo & Botânica & $n_{A}=$ Ana \\
$n_{A}$ & Álgebra & Zoologia & $n_{A}=$ Ana \\
\hline
\end{tabular}

ou seja, as duas últimas tuplas só pertencem à relação se a condição $n_{A}=$ Ana é válida.

\subsection{Atualização}

Uma atualização em banco de dados com valores nulos está semanticamente correta se a representação da relação atualizada $r^{\prime}$ é o mesmo conjunto de relações obtido se atualizamos cada uma das relações originais [ZP92]. Por exemplo, a inserção de uma tupla $t$ em uma relação $r$ é equivalente a inserir $t$ em cada $r_{j} \in \operatorname{Rep}(r)$. A figura seguinte ilustra essa situação :

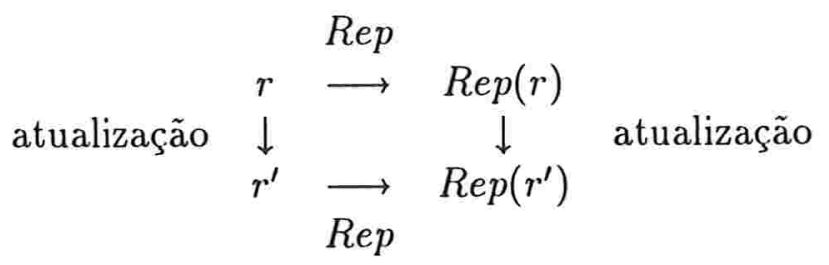

Atualizações em bancos de dados com valores nulos têm ainda uma propriedade adicional: podem ser especificadas com valores nulos, representando uma atualização "incompleta". Por exemplo, considere a relação Lecional a seguir:

Leciona1
\begin{tabular}{|c|c|}
\hline Professor & Discip \\
\hline \hline Ana & Álgebra \\
$n_{A}$ & Geometria \\
\hline
\end{tabular}

onde o domínio do atributo Professor é $\{$ Ana, Carlos $\}$ e o domínio do atributo Discip é $\{$ Álgebra, Geometria, Cálculo $\}$. Seja a atualização At= "Carlos leciona uma disciplina, mas não se sabe qual". Temos então a seguinte situação:

- $\operatorname{Rep}($ Leciona 1$)=r_{L 1}, r_{L 2}$ 
- $\operatorname{Rep}(\mathrm{At})=r_{A t 1}, r_{A t 2}, r_{A t 3}$

onde:

\begin{tabular}{|c|c|}
\multicolumn{2}{c}{$r_{L 1}$} \\
\hline Professor & Discip \\
\hline \hline Ana & Álgebra \\
Ana & Geometria \\
\hline
\end{tabular}

\begin{tabular}{|c|c|}
\multicolumn{2}{c}{$r_{L 2}$} \\
\hline Professor & Discip \\
\hline \hline Ana & Álgebra \\
Carlos & Geometria \\
\hline
\end{tabular}

\begin{tabular}{|c|c|}
\multicolumn{2}{c}{$r_{\text {At1 }}$} \\
\hline Professor & Discip \\
\hline \hline Carlos & Álgebra \\
\hline
\end{tabular}

\begin{tabular}{|c|c|}
\multicolumn{2}{c}{$r_{\text {At2 }}$} \\
\hline Professor & Discip \\
\hline \hline Carlos & Geometria \\
\hline
\end{tabular}

$r_{A t 3}$

\begin{tabular}{|c|c|}
\hline Professor & Discip \\
\hline \hline Carlos & Cálculo \\
\hline
\end{tabular}

ou seja, uma atualização com valores nulos também representa uma série de possibilidades. O resultado final da atualização é calculado através de operações entre conjuntos de possibilidades. No exemplo, a relação atualizada fica:

Leciona2

\begin{tabular}{|c|c|}
\hline Professor & Discip \\
\hline \hline Ana & Álgebra \\
$n_{A}$ & Geometria \\
Carlos & $n_{A}$ \\
\hline
\end{tabular}

Com as seguintes possibilidades em Rep(Leciona2):

\begin{tabular}{|c|c|}
\hline Professor & Discip \\
\hline \hline Ana & Álgebra \\
Ana & Geometria \\
Carlos & Álgebra \\
\hline
\end{tabular}

\begin{tabular}{|c|c|}
\hline Professor & Discip \\
\hline \hline Ana & Álgebra \\
Ana & Geometria \\
Carlos & Geometria \\
\hline
\end{tabular}

\begin{tabular}{|c|c|}
\hline Professor & Discip \\
\hline \hline Ana & Álgebra \\
Ana & Geometria \\
Carlos & Cálculo \\
\hline
\end{tabular}

\begin{tabular}{|c|c|}
\hline Professor & Discip \\
\hline \hline Ana & Álgebra \\
Carlos & Geometria \\
Carlos & Cálculo \\
\hline
\end{tabular}




\begin{tabular}{|c|c|}
\hline Professor & Discip \\
\hline \hline Ana & Álgebra \\
Carlos & Geometria \\
Carlos & Álgebra \\
\hline
\end{tabular}

\begin{tabular}{|c|c|}
\hline Professor & Discip \\
\hline \hline Ana & Álgebra \\
Carlos & Geometria \\
\hline
\end{tabular}

As operações usuais de atualização (inserção, alteração e remoção) não são, portanto, suficientes para expressar atualizações de modo geral em bancos de dados com valores nulos. Abiteboul e Grahne [AG85] descrevem diversos tipos de atualização nesses bancos, e a definição de seu significado em termos das possibilidades que o banco representa. Apresentamos a seguir estas idéias.

\subsubsection{Inserção}

O resultado de uma operação de inserção generalizada, isto é, onde a inserção pode não ser totalmente especificada, é obtido inserindo-se em cada uma das possibilidades, representadas pela relação inicial, cada uma das possibilidades representadas pela inserção. O exemplo de atualização anterior, que transforma a relação Lecional em Leciona2, é uma inserção generalizada. A definição dessa operação é feita através do conceito de união em pares:

$$
\operatorname{Rep}(r)(\cup) \operatorname{Rep}(s)=\left\{r_{i} \cup s_{j} \mid r_{i} \in \operatorname{Rep}(r) \text { e } s_{j} \in \operatorname{Rep}(s)\right\}
$$

Uma inserção Ins em uma relação $r$ pode ser descrita da forma $\operatorname{Rep}(r)(\mathrm{U}) \operatorname{Rep}(\operatorname{Ins})$.

\subsubsection{Remoção}

O conceito de remoção generalizada é análogo ao da inserção generalizada, pois as possibilidades representadas pela remoção devem ser retiradas de cada uma das possibilidades da relação inicial. A definição desse operador é feita através do conceito de diferença em pares:

$$
\operatorname{Rep}(r)(-) \operatorname{Rep}(s)=\left\{r_{i}-r_{j} \mid r_{i} \in \operatorname{Rep}(r) \text { e } s_{j} \in \operatorname{Rep}(s)\right\}
$$

Por exemplo, o resultado de "remover todos os fatos a respeito de Carlos" na relação Leciona2 é obtido calculando $X(-) Y$, onde $X=\operatorname{Rep}$ (Leciona2) e $Y=$ $\operatorname{Rep}\left(\left(\operatorname{Carlos}, n_{A}\right)\right)$, como ilustrado na figura a seguir: 


\begin{tabular}{|c|c|}
\hline Professor & Discip \\
\hline \hline Ana & Álgebra \\
Ana & Geometria \\
\hline
\end{tabular}

\begin{tabular}{|c|c|}
\hline Professor & Discip \\
\hline \hline Ana & Álgebra \\
\hline
\end{tabular}

\subsubsection{Integração}

O conhecimento representado por dois bancos de dados incompletos $B_{1}$ e $B_{2}$ pode ser integrado tomando-se as tuplas comuns às possibilidades representadas por $\operatorname{Rep}\left(B_{1}\right)$ e $\operatorname{Rep}\left(B_{2}\right)$. A definição do operador de integração é feita através do conceito de interseç̧ão aos pares:

$$
\operatorname{Rep}(r)(\cap) \operatorname{Rep}(s)=\left\{r_{i} \cap s_{j} \mid r_{i} \in \operatorname{Rep}(r) \text { e } s_{j} \in \operatorname{Rep}(s)\right\}
$$

Por exemplo, se integramos à relação Lecional a seguinte relação:

\begin{tabular}{|c|c|}
\multicolumn{2}{c}{ Leciona3 } \\
\hline Professor & Discip \\
\hline \hline Ana & Álgebra \\
Ana & Geometria \\
$n_{A}$ & Geometria \\
\hline
\end{tabular}

devemos calcular Rep(Leciona1) (ח) Rep(Leciona3), cujo resultado é:

\begin{tabular}{|c|c|}
\hline Professor & Discip \\
\hline \hline Ana & Álgebra \\
Ana & Geometria \\
\hline
\end{tabular}$\quad$\begin{tabular}{|c|c|} 
Professor & Discip \\
\hline \hline Ana & Álgebra \\
Carlos & Geometria \\
\hline
\end{tabular}

\begin{tabular}{|c|c|}
\hline Professor & Discip \\
\hline \hline Ana & Álgebra \\
\hline
\end{tabular}

\subsubsection{Sujeição}

O conhecimento representado por uma relação $r$ pode tornar-se mais preciso, no sentido em que, obtendo mais informações a respeito do mundo real, temos menos 
possibilidades em $\operatorname{Rep}(r)$. Por exemplo, considere a relação Leciona2, e suponha que se saiba que Ana leciona no máximo uma disciplina. Nesse caso, as possibilidades em Rep(Leciona2) ficam apenas:

\begin{tabular}{|c|c|}
\hline Professor & Discip \\
\hline \hline Ana & Álgebra \\
Carlos & Geometria \\
Carlos & Álgebra \\
\hline
\end{tabular}

\begin{tabular}{|c|c|}
\hline Professor & Discip \\
\hline \hline Ana & Álgebra \\
Carlos & Geometria \\
Carlos & Cálculo \\
\hline
\end{tabular}

\begin{tabular}{|c|c|}
\hline Professor & Discip \\
\hline \hline Ana & Álgebra \\
Carlos & Geometria \\
\hline
\end{tabular}

Se tomamos como $Y$ o conjunto de todas as instâncias possíveis onde Ana leciona apenas uma disciplina, podemos definir a atualização acima como $\operatorname{Rep}($ Leciona2) $\cap$ $Y$.

Esta operação de atualização é chamada sujeição. Intuitivamente, estamos tomando a relação inicial e sujeitando-a a uma série de restrições adicionais.

\subsubsection{Sujeição negativa}

Analogamente à sujeição pode se incorporar conhecimentos negativos. Por exemplo, considere a relação Leciona2 e suponha que se saiba que Carlos não leciona Álgebra. Nesse caso, as possibilidades em $\operatorname{Rep}($ Leciona2) ficam:

\begin{tabular}{|c|c|}
\hline Professor & Discip \\
\hline \hline Ana & Álgebra \\
Ana & Geometria \\
Carlos & Geometria \\
\hline
\end{tabular}

\begin{tabular}{|c|c|}
\hline Professor & Discip \\
\hline \hline Ana & Álgebra \\
Carlos & Geometria \\
\hline
\end{tabular}

\begin{tabular}{|c|c|}
\hline Professor & Discip \\
\hline \hline Ana & Álgebra \\
Ana & Geometria \\
Carlos & Cálculo \\
\hline
\end{tabular}

\begin{tabular}{|c|c|}
\hline Professor & Discip \\
\hline \hline Ana & Álgebra \\
Carlos & Geometria \\
Carlos & Cálculo \\
\hline
\end{tabular}


Nesse caso, a nova relação Leciona2' deve ser tal que Rep(Leciona2') $=$ Rep (Leciona2)-Y, onde Y é o conjunto de todas as instâncias onde Carlos leciona álgebra. Em outras palavras, retiramos de Rep(Leciona2) as relações onde existia a tupla (Carlos, Álgebra).

\subsubsection{Adição}

A adição de um banco de dados $B_{1}$ a outro banco $B_{2}$ é definido por $\operatorname{Rep}\left(B_{1}\right) \cup \operatorname{Rep}\left(B_{2}\right)$. $\mathrm{O}$ significado intuitivo da operação é que com o conhecimento em $B_{1}$, considerávamos possível cada $r \in R e p\left(B_{1}\right)$, e agora, com a adição de $B_{2}$, consideramos possíveis também as relações em $\operatorname{Rep}\left(B_{2}\right)$.

\subsubsection{Modificação}

A modificação é uma generalização do operador de alteração de uma tupla. Para "modificar" uma relação com valores nulos $r$ a modificação deve ser feita em cada uma das possibilidades em $\operatorname{Rep}(r)$. Formalmente, a modificação é uma função $f$ de relações em relações. Quando aplicado à representação $\operatorname{Rep}(r), f$ a transforma em $f(\operatorname{Rep}(r))$ definida por $f(\operatorname{Rep}(r))=\left\{f\left(r_{i}\right) \mid r_{i} \in \operatorname{Rep}(r)\right\}$. Considere a representação da relação Lecional e a seguinte modificação $f$ : "mudar a disciplina que Carlos leciona para Álgebra". O resultado $f(\operatorname{Rep}($ Leciona1)) seria:

\begin{tabular}{|c|c|}
\hline Professor & Discip \\
\hline \hline Ana & Álgebra \\
Ana & Geometria \\
\hline
\end{tabular}

\begin{tabular}{|c|c|}
\hline Professor & Discip \\
\hline \hline Ana & Álgebra \\
Carlos & Álgebra \\
\hline
\end{tabular}

Note que, assim como outras atualizações, a modificação pode ser parcialmente especificada. Por exemplo, pode-se mudar a disciplina que Carlos leciona para Álgebra ou Cálculo. Isto é feito através de uma modificação $F$ que é o conjunto de 2 modificações: uma idêntica à do exemplo anterior e outra que modifica a disciplina de Carlos para Cálculo. O resultado de uma modificação como essa é definido por $F(\operatorname{Rep}(r))=\left\{f\left(r_{i}\right) \mid f \in F \wedge r_{i} \in \operatorname{Rep}(r)\right\}$. No exemplo, a modificação é obtida adicionando-se a seguinte relação às possibilidades do exemplo anterior:

\begin{tabular}{|c|c|}
\hline Professor & Discip \\
\hline \hline Ana & Álgebra \\
Carlos & Cálculo \\
\hline
\end{tabular}


A descrição desses operadores de atualização foi feita em termos de operações sobre as possibilidades que o banco de dados representa. É necessário desenvolver uma forma sintática de atualizar os bancos de dados com valores nulos de forma a manter a mesma semântica das possibilidades repreentadas. Em [ZP92], são apresentadas algumas dificuldades que aparecem ao se tentar definir atualizações em bancos de dados incompletos. Por exemplo, considere as relações Leciona e Matriculado a seguir:

Leciona

\begin{tabular}{|c|c|}
\hline Professor & Discip \\
\hline \hline Ana & Cálculo \\
\hline
\end{tabular}

Matriculado

\begin{tabular}{|c|c|c|}
\hline Aluno & Discip & Depto \\
\hline \hline Juca & Cálculo & Química \\
Marcos & $n_{A}$ & Botânica \\
Pedro & $n_{A}$ & Física \\
\hline
\end{tabular}

Suponha que se deseja alterar o banco de dados de forma que todos os alunos matriculados em Cálculo sejam do departamento de Botânica. A tupla (Juca, Cálculo, Botânica) certamente pertence à relação atualizada, mas para a tupla (Pedro, $n_{A}$, Física) o problema é que o valor do departamento vai depender do valor que assumir o valor nulo $n_{A}$. Esta situação pode ser representada através da seguinte C-tabela:

\begin{tabular}{|c|c|c|c|}
\multicolumn{4}{c|}{ Matriculado } \\
\hline Aluno & Discip & Depto & Condição \\
\hline \hline Juca & Cálculo & Botânica & \\
Marcos & $n_{A_{1}}$ & Botânica & \\
Pedro & $n_{A_{2}}$ & Física & $n_{A_{2}} \neq$ Cálculo \\
Pedro & $n_{A_{2}}$ & Botânica & $n_{A_{2}}=$ Cálculo \\
\hline
\end{tabular}

Considere agora uma atualização onde ou Marcos ou Pedro é removido da relação Matriculado (apenas um deles é removido). Intuitivamente, isto significa que, em cada possibilidade em $\operatorname{Rep}$ (Matriculado), uma das tuplas (e somente uma) é removida. A relação atualizada pode ser representada pela seguinte C-tabela:

\begin{tabular}{|c|c|c|c|}
\multicolumn{4}{c|}{ Matriculado } \\
\hline Aluno & Discip & Depto & Condição \\
\hline Juca & Cálculo & Botânica & \\
Marcos & $n_{A_{1}}$ & Botânica & $n_{A_{3}} \neq$ Marcos \\
Pedro & $n_{A_{2}}$ & Física & $n_{A_{3}} \neq$ Pedro \\
\hline
\end{tabular}

com uma condição global garantindo que $\left(n_{A_{3}}=\right.$ Marcos $\vee n_{A_{3}}=$ Pedro), ou seja, o valor vazio $n_{A_{3}}$ assume um (e somente um) dos valores Marcos ou Pedro. 
Os exemplos apresentados ilustram o resultado obtido em [AG85], que afirmam que através de C-tabelas com condições globais é possível representar todos os resultados das operações de atualização introduzidos. Usando somente relações com valores nulos, isto não é possível.

Em [Var86], é analisada a complexidade computacional da verificação de integridade em bancos de dados incompletos. O resultado interessante é que, de modo geral, as operações de atualização com verificação de integridade têm um custo maior quando se assume a hipótese do mundo fechado (CWA). Na verdade, enquanto sob a hipótese do mundo aberto (OWA) este problema é resolvido em tempo polinomial, sob a CWA o problema pertence à classe NP. Outra análise interessante é que a situação se inverte se consideramos operações de consulta: sob a CWA, a complexidade dessas operações é a mesma de consultas em bancos de dados convencionais, mas sob a OWA, o problema é intratável. Mais detalhes sobre a OWA e a CWA são apresentados no capítulo 8 .

\subsection{Atualizações em representação lógica}

As operações de atualização descritas na seção anterior são consideradas mapeamentos de bancos de dados em bancos de dados. Outra abordagem para descrever essas operações é através da representação lógica de bancos de dados, via Teoria de Prova (Cap.5). O trabalho em [Win88] é feito nesse sentido e apresentamos aqui as principais idéias e resultados encontrados.

Conforme vimos no capítulo 5, na representação lógica o banco de dados com valores nulos é representado através de uma série de fórmulas em lógica de primeira ordem chamadas de teoria relacional estendida. Para cada banco de dados existem um ou mais modelos. Em uma analogia com a representação via álgebra relacional, a teoria é como a representação sintática do banco de dados - através de relações com valores nulos, C-tabelas ou outra representação tabular - e os modelos são as diversas possibilidades representadas. Nesse sentido, o significado de atualização correta não se altera - toda atualização feita na teoria deve refletir-se adequadamente nos modelos.

Uma atualização na representação lógica é algo da forma:

$$
\text { insert (nova-fórmula) where (antiga-fórmula) }
$$

Os demais operadores de atualização podem ser escritos nessa mesma forma, como ilustram os exemplos a seguir, considerando os predicados Funcionário(Nome, Depto) 
e Gerente(Nome,Depto):

- insert Funcionário(Prado, MAC) $\vee$ Funcionário(Prado, MAT)

where Gerente (Nilson, MAT)

Nos modelos onde Nilson é gerente do departamento MAT, inclua o fato que Prado é funcionário do departamento MAT, ou do MAC, ou de ambos.

- insert $\neg$ Funcionário(Prado, MAC)

where $(\neg$ Gerente(Nilson, MAC) $\wedge$ Funcionário(Prado, MAC))

Nos modelos onde Nilson não é gerente do MAC , remova o fato que Prado é funcionário do MAC.

- insert $\mathbf{F}$ (falso)

where $\neg$ Funcionário(Prado, MAT)

Elimine todos os modelos onde Prado não é funcionário do MAT. No caso, ao inserirmos uma fórmula sempre falsa, o resultado é que o antigo modelo passa a não ser mais modelo de nenhum conjunto de fórmulas.

- insert $\neg$ Funcionário(Prado,MAC) $\wedge$ Funcionário(Prado,MAT)

where Funcionário(Prado,MAC)

Nos modelos onde Prado é funcionário do MAC, mude seu departamento para MAT.

Note que a remoção é feita através da inclusão do fato negado. Isto é equivalente a remover o fato, pois um provador de teoremas, utilizando a hipótese do mundo fechado, tem duas formas de concluir um fato negado $\neg \mathrm{P}$ :

- quando o fato $\neg \mathrm{P}$ é um teorema da teoria, isto é, $\vdash \neg \mathrm{P}$, ou

- quando o fato $\mathrm{P}$ não é um teorema da teoria, isto é, $\forall \mathrm{P}$.

A partir da definição da sintaxe e semântica de atualizações, é desenvolvido um algoritmo para efetuar essas atualizações na teoria. A idéia básica do algoritmo é a seguinte: seja $A=$ (insert novo-f where antigo-f) uma atualização em um banco de dados representado pela teoria $\mathrm{T}$.

1. Para cada fórmula atômica $f_{a}$ em $\mathrm{T}$ que aparece em novo-f, trocar todas as ocorrências de $f_{a}$ por uma fórmula do tipo Histórico $\left(f_{a}, \mathrm{~A}\right)$, indicando que $f_{a}$ estava na teoria $\mathrm{T}$ antes da atualização $\mathrm{A}$ ser efetuada. 
2. Adicionar uma nova fórmula a $\mathrm{T}$ que define o valor correto de $f_{a}$ (F ou $\mathrm{V}$ ) quando a fórmula antigo-f é falsa.

3. Adicionar uma nova fórmula a $\mathrm{T}$ que define o valor correto de $f_{a}(\mathrm{~F}$ ou $\mathrm{V})$ quando a fórmula antigo-f é verdadeira.

O algoritmo apresentado é correto em relação à semântica especificada. 


\section{Capítulo 8}

\section{Negação}

A terminologia "hipótese do mundo fechado" (CWA, de "Closed World Assumption") foi introduzida por Reiter [Rei78], para se referir à suposição de que toda informação não representada explicitamente no banco de dados é falsa. Por exemplo, considere a relação Funcionários a seguir:

\begin{tabular}{|l|l|}
\hline Funcionário & Depto \\
\hline \hline Mario & Pessoal \\
Paulo & Pessoal \\
Lucia & Contabilidade \\
\hline
\end{tabular}

Se assumimos a hipótese do mundo fechado, a resposta à consulta "Mario trabalha no departamento de contabilidade" é não. Essa hipótese estabelece também que não existem outros funcionários além de Mario, Paulo e Lucia, e que não existem outros departamentos além de Pessoal e Contabilidade

Outra forma de se interpretar a informação contida no banco de dados é assumir que este representa uma parte (conhecida) das informações existentes, e que podem existir outras informações além das descritas explicitamente. Intuitivamente, é esta idéia da hipótese do mundo aberto (OWA, de "Open World Assumption"). No exemplo anterior, se assumimos essa hipótese, a resposta à consulta é não sei, e não se sabe se existem outros funcionários além de Mario, Paulo e Lucia ou outros departamentos além de Pessoal e Contabilidade.

Quando se adota a OWA, os fatos negativos devem ser explicitamente representados no banco de dados. Por exemplo, para representar o fato "Ana não é funcionária 
do departamento Pessoal", temos que separar a relação Funcionários em duas componentes: Positiva e Negativa, como descrito em [ZP92]. A relação fica na forma:

Funcionários

\begin{tabular}{|c|c|c|}
\hline Funcionário & Depto & \\
\hline Mario & Pessoal & \\
\hline Paulo & Pessoal & parte positiva \\
\hline Lucia & Contabilidade & \\
\hline Ana & Pessoal & parte negativa \\
\hline
\end{tabular}

Utilizando representação em lógica de primeira ordem, a teoria deve conter os axiomas Funcionário(Mario, Pessoal), Funcionário(Paulo, Pessoal), Funcionário(Lucia, Contabilidade) e $\neg$ Funcionário(Ana, Pessoal), além dos axiomas que traduzem os conceitos de nomes únicos. $\mathrm{O}$ axioma do completamento para a relação Funcionários é invertido para traduzir o fato de que podem haver mais tuplas na relação:

$$
\begin{aligned}
\forall x \forall y[\quad & (x=\text { Mario } \wedge y=\text { Pessoal }) \vee \\
& (x=\text { Paulo } \wedge y=\text { Pessoal }) \vee \\
& (x=\text { Lucia } \wedge y=\text { Contabilidade }) \Rightarrow \text { Funcionário }(x, y)]
\end{aligned}
$$

É interessante notar que o mesmo efeito é obtido se permitimos o uso de valores nulos e tuplas onde todos os atributos são valores nulos, isto é, se temos o axioma:

$$
\begin{aligned}
\forall x \forall y \text { Funcionário }(x, y) \Rightarrow \quad & (x=\text { Mario } \wedge y=\text { Pessoal }) \vee \\
& (x=\text { Paulo } \wedge y=\text { Pessoal }) \vee \\
& (x=\text { Lucia } \wedge y=\text { Contabilidade }) \vee \\
& \left(x=n_{A_{1}} \wedge y=n_{A_{2}}\right)
\end{aligned}
$$

onde $n_{A_{1}}$ e $n_{A_{2}}$ são valores nulos.

Para se decidir qual dessas hipóteses (OWA ou CWA) será adotada deve ser considerado o tipo de aplicação que utiliza o banco de dados. A OWA representa mais fielmente a realidade, no sentido de que o banco de dados não contém todas as informações e este fato é levado em consideração. No entanto, para muitas aplicações práticas, a CWA é mais adequada. O cadastro de funcionários de uma empresa é um bom exemplo. Obviamente, é mais razoável assumir que as pessoas que não constam no cadastro não são funcionários do que armazenar componentes negativas de todas as outras pessoas do mundo. 
A regra de inferência de negação por falha finita, analisada em [Cla78] e que a linguagem Prolog utiliza para tratar negação também adota a hipótese do mundo fechado, no sentido em que se permite concluir $\neg \mathrm{P}$ quando não se consegue uma prova para $\mathrm{P}$.

\subsection{Hipótese do mundo fechado estendida}

Em bancos de dados com valores nulos, a hipótese do mundo fechado pode ocasionar inconsistências. Por exemplo, considere a seguinte relação:

Funcionários

\begin{tabular}{|l|l|}
\hline Funcionário & Depto \\
\hline \hline Mario & Pessoal \\
Paulo & Contabilidade \\
Ana & $n_{A}$ \\
\hline
\end{tabular}

Se assumimos a CWA, só existem os departamentos Pessoal e Contabilidade. Supomos também que Ana não pertence ao departamento Pessoal nem ao departamento de Contabilidade, pois estes fatos não estão explicitamente representados na relação. Chegamos então a uma contradição, pois o valor $n_{A}$ na tupla (Ana, $n_{A}$ ) não pode assumir nenhum dos (únicos) valores possíveis no domínio do atributo Departamento.

Minker [Min82] notou essa deficiência na CWA e propôs uma versão estendida que chamou de hipótese do mundo fechado generalizada (GCWA, de "Generalized Closed World Assumption"). A definição da GCWA é feita a partir da representação lógica de bancos de dados onde o valor nulo $n_{A}$ pode ser visto como uma disjunção de elementos no domínio do atributo. No exemplo, a tupla (Ana, $n_{A}$ ) é representada por Funcionário(Ana, Pessoal) $\vee$ Funcionário(Ana, Contabilidade). A idéia básica da GCWA é assumir falsas somente as tuplas que não aparecem em nenhuma das possibilidades representadas pelo banco de dados.

A GCWA também assume, assim como a CWA, que os elementos do domínio dos atributos são apenas aqueles que aparecem explicitamente na relação. Dessa forma, o método para se identificar os fatos assumidos falsos pela GCWA em uma dada relação $r$ é o seguinte:

1. Construir uma relação $H(r)$, de todas as tuplas possíveis na relação original, considerando o domínio de cada atributo como sendo formado apenas por elementos que aparecem explicitamente na relação (a menos de valores nulos), e substituindo os valores nulos por elementos desse domínio fechado. 
2. Construir o conjunto das relações em $\operatorname{Rep}(r)$.

3. Construir o conjunto $\operatorname{RepMin}(r)$, removendo de $\operatorname{Rep}(r)$ as relações contidas em outras relações, isto é, $\operatorname{Rep} \operatorname{Min}(r)=\operatorname{Rep}(r)-\left\{r_{1} \in \operatorname{Rep}(r) \mid \exists r_{2} \in \operatorname{Rep}(r)\left[r_{1} \subseteq\right.\right.$ $\left.\left.r_{2}\right]\right\}$

4. As tuplas assumidas falsas são as que aparecem no conjunto $\overline{\operatorname{Rep}}(r)=\bigcap_{i}(H(r)-$ $\left.r_{i}\right)$, onde $r_{i} \in \operatorname{RepMin}(r)$

No exemplo anterior, para a relação Funcionários, temos:

\begin{tabular}{|l|l|}
\multicolumn{2}{c}{$\mathrm{H}$ (Funcionários) } \\
\hline Funcionário & Depto \\
\hline \hline Mario & Pessoal \\
Mario & Contabilidade \\
Paulo & Pessoal \\
Paulo & Contabilidade \\
Ana & Pessoal \\
Ana & Contabilidade \\
\hline
\end{tabular}

$\operatorname{RepMin}($ Funcionários $)=\operatorname{Rep}($ Funcionários $)=\left\{r_{1}, r_{2}\right\}$, onde:

\begin{tabular}{|l|l|}
\multicolumn{2}{c}{} \\
\hline Funcionário & Depto \\
\hline \hline Mario & Pessoal \\
Paulo & Contabilidade \\
Ana & Pessoal \\
\hline
\end{tabular}

\begin{tabular}{|l|l|}
\multicolumn{2}{c}{$r_{2}$} \\
\hline \hline Funcionário & Depto \\
\hline \hline Mario & Pessoal \\
Paulo & Contabilidade \\
Ana & Contabilidade \\
\hline
\end{tabular}

$\overline{R e p}($ Funcionários $)=\left(\mathrm{H}(\right.$ Funcionários $\left.)-r_{1}\right) \cap\left(\mathrm{H}(\right.$ Funcionários $\left.)-r_{2}\right)$, ou seja:

\begin{tabular}{|l|l|}
\multicolumn{1}{c|}{$\overline{R e p}($ Funcionários $)$} \\
\hline Funcionário & Depto \\
\hline \hline Mario & Contabilidade \\
Paulo & Pessoal \\
\hline
\end{tabular}

Dessa forma, as tuplas (Ana,Pessoal) e (Ana, Contabilidade) não são assumidas falsas, e não existe a contradição.

Intuitivamente, o conjunto $\overline{R e p}(r)$ contém todas as afirmações que não aparecem em nenhuma das possibilidades em $\operatorname{Re} p(r)$, de forma que, ao assumirmos essas 
afirmações como falsas, não temos nenhuma contradição com as possibilidades representadas pela relação original $r$.

A versão da CWA adotada por Biskup [Bis83] segue esta mesma linha de raciocínio, mas é apresentada de uma forma diferente. Seja $r(\mathrm{R})$ uma uma relação com valores nulos. Se $\bar{t}$ é uma tupla (sem valores nulos) que não pode ser obtida a partir de uma tupla $t$ (com valores nulos) na relação $r$, então assumimos que $\bar{t}$ não é um elemento da relação $r$, e portanto o fato correspondente é falso no mundo real. Biskup também salienta que, ao armazenarmos uma tupla composta apenas de valores nulos, não podemos assumir mais nenhuma tupla $\bar{t}$, e portanto estamos adotando a OWA.

\subsection{Valores universais e valores padrão}

Os valores universais e padrão são categorias de valores nulos necessárias para expressar certos fatos quando assumimos a OWA. Apresentamos a seguir estes conceitos, como em [ZP92]. Suponha que se deseja representar os seguintes fatos, na relação Funcionários abaixo:

Funcionários

\begin{tabular}{|l|l|l|}
\hline Funcionário & Depto & Função \\
\hline \hline Mario & Pessoal & Gerente \\
Paulo & Pessoal & Contador \\
\hline
\end{tabular}

- Ana é telefonista de todos os departamentos

- João não exerce nenhuma função no departamento Pessoal

Se a relação for interpretada segundo a CWA, e os departamentos que aparecem no banco de dados são $\left\{d e p_{1}, \ldots, d e p_{n}\right\}$, para representar o primeiro fato podemos introduzir na relação $n$ tuplas da forma (Ana, dep $p_{i}$,telefonista). O segundo fato já está automaticamente armazenado, pois todos os fatos não explicitamente representados são falsos, a menos que exista uma tupla que entre em contradição, como por exemplo (João,Pessoal,Datilógrafo).

A situação é completamente diferente se assumimos a OWA. Como não conhecemos previamente todos os departamentos, não é possível introduzir as $n$ tuplas como anteriormente. O segundo fato não está mais representado, pois sob a OWA fatos negativos devem ser explicitamente representados. Assim, é necessário usar um valor universal, denotado por $n_{\forall}$, da seguinte forma: 
Funcionários

\begin{tabular}{|c|c|c|c|}
\hline Funcionário & Depto & Função & \multirow[b]{3}{*}{ parte positiva } \\
\hline Mario & Pessoal & Gerente & \\
\hline Paulo & Contabilidade & Contador & \\
\hline Ana & $n_{\forall}$ & Telefonista & \multirow[b]{2}{*}{ parte negativa } \\
\hline João & Pessoal & $n_{\forall}$ & \\
\hline
\end{tabular}

Em lógica de primeira ordem, esta relação é representada pelas seguintes fórmulas:

- $(x=$ Mario $\wedge y=$ Pessoal $\wedge z=$ Gerente $) \vee$

( $x=$ Paulo $\wedge y=$ Contabilidade $\wedge z=$ Contador $) \vee$

$(x=$ Ana $\wedge z=$ Telefonista $) \Rightarrow$ Funcionario $(x, y, z)$

- $\forall z \neg$ Funcionário(João,Pessoal,z)

Os valores padrão representam outra categoria de valores nulos necessária quando se adota a OWA. Suponha que a relação Salários a seguir represente a média anual de salários dos gerentes, e se deseja representar o fato que a média anual de todo gerente, quando não representada explicitamente, é 100000, para o ano de 1993:

\begin{tabular}{|l|l|l|}
\hline \multicolumn{3}{|c}{ Salários } \\
\hline \hline Ano & Funcionário & Salário \\
\hline \hline 1992 & Mario & 100000 \\
1993 & Mario & 110000 \\
1992 & Francisco & 120000 \\
1993 & Francisco & 132000 \\
\hline
\end{tabular}

Se adotamos a CWA, este fato é representado armazenando-se uma tupla para cada gerente distinto de Mario e Francisco. Adotando a OWA, como os gerentes não são todos conhecidos, é necessário um valor nulo padrão, denotado por $n_{*}$. A relação fica então da forma:

\begin{tabular}{|l|l|l|}
\multicolumn{3}{|c}{ Salários } \\
\hline Ano & Funcionário & Salário \\
\hline \hline 1992 & Mario & 100000 \\
1993 & Mario & 110000 \\
1992 & Francisco & 120000 \\
1993 & Francisco & 132000 \\
1993 & $n_{*}$ & 100000 \\
\hline
\end{tabular}


Em lógica de primeira ordem, esta relação é representada pelas seguintes fórmulas:

- $(x=1992 \wedge y=$ Mario $\wedge z=100000) \vee$

$(x=1993 \wedge y=$ Mario $\wedge z=110000) \vee$

$(x=1992 \wedge y=$ Francisco $\wedge z=120000) \vee$

$(x=1993 \wedge y=$ Francisco $\wedge z=132000) \vee$

$(x=1993 \wedge y \neq$ Francisco $\wedge y \neq$ Mario $\wedge z=100000)$

$\Rightarrow \operatorname{Salarios}(x, y, z)$

- $\forall x \forall y \forall z \operatorname{Salários}(x, y, z) \wedge x=1993 \wedge y \neq$ Francisco $\wedge y \neq$ Mario $\Rightarrow z=100000$

O uso de informação padrão é muito importante. Muitas aplicações utilizam diversos parâmetros, nem sempre conhecidos, e muitas vezes são necessárias estimativas sobre as quais são tomadas decisões. É o caso, por exemplo, de aplicações que trabalham no mercado financeiro. A informação padrão é um recurso para tratamento de incerteza, porque define valores "médios" ou "comuns", que, na ausência de informação contrária podem ser assumidos como verdadeiros.

\subsection{Valores abertos}

Até agora estudamos o resultado de se adotar uma dentre duas hipóteses: interpretar o banco de dados de forma totalmente aberta ou totalmente fechada. Uma outra possibilidade é utilizar uma interpretação mista, adotando ora a OWA ora a CWA de acordo com a conveniência. Este método traz mais um tipo de valor nulo, o valor nulo aberto, $n_{\Omega}$, descrito a seguir. As idéias aqui apresentadas estão em [ZP92].

Uma forma de se misturar as duas hipóteses é permitir que cada relação do banco seja interpretada sob a CWA ou a OWA. Esta é a idéia da hipótese de fechamento sensivel (CCA, de "Careful Closure Assumption"). A hipótese do mundo aberto localmente (LOWA, de "Locally Open World Assumption") permite que cada atributo de cada relação no banco de dados possa ser interpretado de maneira diferente. A intepretação padrão das relações é a CWA, mas é possível indicar que um ou mais atributos da mesma sejam intepretados sob a OWA. Para tanto utiliza-se o valor nulo $n_{\Omega}$, como na relação Funcionários1 a seguir:

Funcionários1

\begin{tabular}{|l|l|l|}
\hline Funcionário & Depto & Funçấo \\
\hline \hline Hélio & Pessoal & Gerente \\
$n_{\Omega}$ & Contabilidade & Contador \\
\hline
\end{tabular}


Esta relação representa os seguintes fatos:

- Os únicos departamentos existentes são Pessoal e Contabilidade

- As únicas funções são Gerente e Contador

- No departamento de Contabilidade podem existir zero, um ou mais contadores, que podem ser funcionários que ainda não apareceram explicitamente na relação, ou o próprio Hélio.

- Nenhum outro fato é verdadeiro nesta relação.

Consideremos agora a relação Funcionários2 a seguir:

Funcionários2

\begin{tabular}{|l|l|l|}
\hline Funcionário & Depto & Função \\
\hline \hline Hélio & Pessoal & Gerente \\
$n_{\Omega}$ & $n_{\Omega}$ & $n_{\Omega}$ \\
\hline
\end{tabular}

Os valores abertos indicam que a relação toda é interpretada sob a OWA. O único fato realmente conhecido é que Hélio é gerente do departamento de pessoal. Nenhum fato negativo é assumido. O valor nulo $n_{S}$ é um caso particular de $n_{\Omega}$, assumindo no máximo um valor do domínio. Assim, o valor aberto é menos informativo que os valores nulos $n_{A}$ e $n_{I}$.

A semântica dos valores abertos pode ser especificada da seguinte maneira. Considere a relação Funcionários1, e suponha que o domínio do atributo Funcionário seja $\{$ Fernanda, Hélio\}. Assim, a relação representa as seguintes possibilidades em Rep(Funcionários):

\begin{tabular}{|l|l|l|}
\hline Funcionário & Depto & Função \\
\hline \hline Hélio & Pessoal & Gerente \\
$n_{I}$ & Contabilidade & Contador \\
\hline
\end{tabular}

\begin{tabular}{|l|l|l|}
\hline Funcionário & Depto & Função \\
\hline \hline Hélio & Pessoal & Gerente \\
Hélio & Contabilidade & Contador \\
\hline
\end{tabular}




\begin{tabular}{|l|l|l|}
\hline Funcionário & Depto & Função \\
\hline \hline Hélio & Pessoal & Gerente \\
Fernanda & Contabilidade & Contador \\
\hline
\end{tabular}

\begin{tabular}{|l|l|l|}
\hline Funcionário & Depto & Função \\
\hline \hline Hélio & Pessoal & Gerente \\
Hélio & Contabilidade & Contador \\
Fernanda & Contabilidade & Contador \\
\hline
\end{tabular}




\section{Capítulo 9}

\section{Tópicos especiais}

O objetivo deste capítulo é apresentar a utilização de valores nulos em outros modelos de dados: o modelo relacional não normalizado e o modelo orientado a objetos. Estes modelos têm sido objeto de pesquisa recente, na tentativa de representar a realidade de forma mais natural. Os valores nulos aparecem aqui de diversas formas, e são essenciais para a representação de informação incompleta.

\subsection{O modelo relacional não normalizado}

Para representar informações de uma forma mais natural, muitas vezes é necessário empregar objetos mais complexos. O modelo relacional não normalizado (Codd [Codd70], em seu primeiro artigo sobre o modelo relacional, utilizava o termo "normalizado" para se referir às relações onde os atributos eram valores simples) permite que cada atributo da relação seja não apenas uma valor simples, mas conjuntos de valores ou mesmo uma relação "encaixada" em outra. A não normalização, no caso, está no fato que as relações não estão, necessariamente, na Primeira Forma Normal [Codd72]. As idéias apresentadas nesta seção estão em [RKS89] e [ZP92].

O modelo relacional não normalizado é mais adequado para diversas aplicações, pois permite representar mais facilmente certos fatos. Por exemplo, considere a relação Livros a seguir [Set89]: 
Livros

\begin{tabular}{|c|c|c|c|c|}
\hline Código & Autores & Título & Assuntos & Ano \\
\hline 100 & $\begin{array}{l}\text { Curtice, R.M. } \\
\text { Jones, P.E. }\end{array}$ & $\begin{array}{c}\text { Logical } \\
\text { DB design }\end{array}$ & $\begin{array}{l}\text { Bancos de dados } \\
\text { Mod.Conceitual }\end{array}$ & 1982 \\
\hline 200 & $\begin{array}{l}\text { Setzer, V.W. } \\
\text { Melo, I.S.H. }\end{array}$ & $\begin{array}{l}\text { A construção de } \\
\text { um compilador }\end{array}$ & $\begin{array}{l}\text { Compilação } \\
\text { An. Sintática } \\
\text { Estr. de Dados }\end{array}$ & 1985 \\
\hline
\end{tabular}

Para cada livro, armazenamos em sua tupla todos os dados a seu respeito. Nesse caso, esta forma de representação é mais natural do que utilizar três relações em Primeira Forma Normal, uma para os livros, outra para assuntos e outra para autores:

Livros

\begin{tabular}{|l|l|l|}
\hline Codigo & Título & Ano \\
\hline \hline 100 & Logical DB design & 1982 \\
200 & A construção de um compilador & 1985 \\
\hline
\end{tabular}

\begin{tabular}{|l|l|}
\multicolumn{2}{c}{ Autores } \\
\hline Código & Autor \\
\hline \hline 100 & Curtice, R.M. \\
100 & Jones, P.E. \\
200 & Setzer, V.W. \\
200 & Melo, I.S.H. \\
\hline
\end{tabular}

\begin{tabular}{|l|l|}
\multicolumn{2}{c}{ Assuntos } \\
\hline Código & Assunto \\
\hline \hline 100 & Bancos de dados \\
100 & Mod. Conceitual \\
200 & Compilação \\
200 & An. Sintática \\
200 & Estr. de dados \\
\hline
\end{tabular}

A representação não-normalizada corresponde a uma visão "esperada" pelo usuário e simplifica as consultas, tornando-as também mais eficientes.

Com o uso desses atributos mais complexos, foi criada a necessidade de rever os fundamentos da teoria de bancos de dados relacionais, para operar sobre relações não normalizadas. Além disso, foram introduzidos operadores que utilizam a estrutura não normalizada da relação.

No modelo relacional não normalizado, os atributos de uma relação não são os valores que Codd [Codd70] denominou de "atômicos". O esquema de uma relação no modelo não normalizado é uma sequência de atributos $R=\left(A_{1}, \ldots, A_{n}\right)$. A cada 
atributo $A_{i}$ está associado um Domínio, $\operatorname{Dom}\left(A_{i}\right)$. Cada elemento de $\operatorname{Dom}\left(A_{i}\right)$ pode ser, indutivamente:

- um valor atômico $c$

- um conjunto de valores $\left\{c_{1}, \ldots, c_{k}\right\}$

- uma relação $r(R) \subseteq B_{1} \times B_{2} \times \ldots \times B_{l}$ onde $\mathrm{R}=\left(B_{1}, \ldots, B_{l}\right)$ é um esquema não normalizado.

Os atributos de ordem zero em uma relação não normalizada são aqueles para as quais o domínio é um conjunto de valores atômicos. Indicamos que um atributo $A$ é de ordem maior que zero denotando-o por $A^{*}$. Dizemos que uma relação $r(R)$ não normalizada está na Forma Normal Particionada [RKS89] se :

- os atributos de ordem zero formam uma chave de $r$.

- os atributos de ordem maior que zero (as relações "encaixadas") estão na Forma Normal Particionada

O modelo relacional não normalizado possui, além dos operadores usuais da álgebra relacional, dois operadores a mais, nest $(r)$ e unnest $(r)$, introduzidos por Jaeschke e Scheck [JS82]. Intuitivamente, o operador nest transforma a relação $r$ em uma relação $r^{\prime}$ "menos" normalizada, no sentido de possuir mais atributos de ordem maior que zero. O operador unnest, ao contrário, normaliza a relação em um nível, expandindo um desses atributos. O exemplo abaixo ilustra o uso desses operadores:

Funcionários

\begin{tabular}{|c|c|c|c|c|}
\hline \multirow[b]{3}{*}{ Funcionário } & \multicolumn{4}{|c|}{ Dependentes* } \\
\hline & \multirow[b]{2}{*}{ Nome } & \multirow[b]{2}{*}{ Nascimento } & \multicolumn{2}{|c|}{ Vacinas $^{*}$} \\
\hline & & & Vacina & Data \\
\hline \multirow[t]{4}{*}{ Hélio } & Monica & $15 / 05 / 61$ & Polio I & $01 / 01 / 62$ \\
\hline & & & Polio II & $01 / 07 / 62$ \\
\hline & Fernanda & $09 / 11 / 70$ & Sarampo & $01 / 01 / 71$ \\
\hline & & & Polio I & $01 / 01 / 71$ \\
\hline \multirow[t]{3}{*}{ Mario } & Mauricio & $28 / 07 / 70$ & Sarampo & $01 / 01 / 71$ \\
\hline & & & Polio I & $01 / 01 / 71$ \\
\hline & Mariane & $26 / 07 / 63$ & Sarampo & $01 / 01 / 64$ \\
\hline
\end{tabular}

Se aplicamos o operador unnest na relação acima, em relação ao atributo Vacinas*, obtemos: 


\begin{tabular}{|l|l|l|l|l|}
\multicolumn{5}{c|}{ unnest (Funcionários) } \\
\hline \multirow{3}{*}{ Funcionário } & \multicolumn{4}{|c|}{ Dependentes } \\
\cline { 2 - 5 } & Nome & Nascimento & Vacina & Data \\
\hline \hline Hélio & Monica & $15 / 05 / 61$ & Polio I & $01 / 01 / 62$ \\
& Monica & $15 / 05 / 61$ & Polio II & $01 / 07 / 62$ \\
& Fernanda & $09 / 11 / 70$ & Sarampo & $01 / 01 / 71$ \\
& Fernanda & $09 / 11 / 70$ & Polio I & $01 / 01 / 71$ \\
& Mauricio & $28 / 07 / 70$ & Sarampo & $01 / 01 / 71$ \\
& Mauricio & $28 / 07 / 70$ & Polio I & $01 / 01 / 71$ \\
& Mariane & $26 / 07 / 63$ & Sarampo & $01 / 01 / 64$ \\
\hline
\end{tabular}

Inversamente, se aplicamos o operador nest em relação aos atributos (Vacina, Data), ou seja, Vacinas* obtemos a relação anterior.

Note que se aplicamos o operador unnest repetidas vezes (notação: unnest*), obtemos uma relação em Primeira Forma Normal. No exemplo, obtemos:

unnest* (Funcionários)

\begin{tabular}{|l|l|l|l|l|}
\hline Funcionário & Nome & Nascimento & Vacina & Data \\
\hline \hline Hélio & Monica & $15 / 05 / 61$ & Polio I & $01 / 01 / 62$ \\
Hélio & Monica & $15 / 05 / 61$ & Polio II & $01 / 07 / 62$ \\
Hélio & Fernanda & $09 / 11 / 70$ & Sarampo & $01 / 01 / 71$ \\
Hélio & Fernanda & $09 / 11 / 70$ & Polio I & $01 / 01 / 71$ \\
Mario & Mauricio & $28 / 07 / 70$ & Sarampo & $01 / 01 / 71$ \\
Mario & Mauricio & $28 / 07 / 70$ & Polio I & $01 / 01 / 71$ \\
Mario & Mariane & $26 / 07 / 63$ & Sarampo & $01 / 01 / 64$ \\
\hline
\end{tabular}

O problema de valores nulos em relações não normalizadas está ligado à representação do conjunto vazio nessas relações. Por exemplo, suponha que desejamos incluir o funcionário Antonio, sem dependentes, na relação Funcionários. Incluimos então a tupla (Antonio, $\Phi$ ). O operador unnest*, aplicado a essa relação, deve produzir uma tupla do tipo (Antonio, $n, n, n, n$ ), onde $n$ representa um valor nulo. A questão a ser analisada é que tipo de valor nulo deve ser utilizado, ou seja, qual valor nulo conserva a semântica do conjunto vazio original.

Segundo [ZP92], muitos autores afirmam que ao aplicar o operador unnest ao conjunto vazio produzimos um valor nulo indefinido ou não-natural. Alguns atribuem a mesma interpretação do nulo não aplicável $n_{I}$. Em [RKS89], o valor nulo sem informação $n_{S}$ é tido como o mais adequado, interpretando a relação sob a OWA. Zicari [Zic90] também adota o uso do valor nulo $n_{S}$ nesses casos, e afirma que o 
conjunto vazio é um tipo particular de valor nulo. A explicação intuitiva é que o conjunto vazio é um valor do domínio de atributos compostos e não há nenhum valor no domínio dos atributos das relações normalizadas correspondentes que o represente, portanto somos obrigados a incluir o valor nulo $n_{S}$.

No exemplo do funcionário Antonio, a tupla

\section{Antonio $\Phi$}

em uma relação normalizada é expandida para

\begin{tabular}{|l|l|l|l|l|}
\hline Antonio & $n_{S}$ & $n_{S}$ & $n_{S}$ & $n_{S}$ \\
\hline
\end{tabular}

Ao utilizarmos o valor nulo $n_{S}$ estamos representando o fato de que não sabemos se Antonio tem ou não dependentes. Por outro lado, se utilizarmos o valor nulo $n_{I}$, afirmamos que Antonio não possui dependentes. No primeiro caso, atribuímos ao conjunto vazio inicial a interpretação "não se possui informação sobre os dependentes de Antonio", e, no segundo, "não existem dependentes de Antonio".

Em bancos de dados normalizados, os valores nulos são utilizados para representar algum tipo de informação incompleta. Esse, porém, não é sempre o caso em modelos não normalizados. O exemplo apresentado em [RKS89] ilustra este fato: considere um banco de dados com as relações Leciona(Professor, Disciplina) e Pesquisa(Professor, Projeto). Suponha que a relação Leciona contenha várias tuplas correspondentes às disciplinas lecionadas pelo Prof. Silva, e que ele não participe de nenhum projeto de pesquisa. Esse banco de dados pode ser representado por uma relação não normalizada com atributos Professor (ordem zero), Disciplina e Projeto (ordem maior que zero). Nesse caso, a tupla correspondente ao Prof. Silva tem um conjunto vazio no atributo Projeto. Através desse exemplo vemos que o uso de valores nulos é ainda mais necessário em relações não normalizadas.

O trabalho desenvolvido em [RKS89] estende a álgebra relacional para relações não normalizadas com valores nulos. Segundo [ZP92], a base desse trabalho está em [Zan84], que define os valores nulos aplicável, inaplicável e sem informação. Entretanto, não são considerados os valores nulos identificados, que permitem a representação mais adequada de informação incompleta, especialmente para tratar dependências. 


\subsection{Bancos de dados orientados a objetos}

A orientação a objetos é uma técnica recente de programação e organização de dados que indentifica objetos e atribui a estes estrutura e comportamento específicos, além de definir o inter-relacionamento entre objetos. Dessa forma, permite uma organização mais precisa e segura dos dados e seu comportamento. Em bancos de dados, a orientação a objetos é um passo a mais em direção à solução do problema de verificação de integridade dos dados na atualização do banco.

A seguir apresentamos alguns conceitos básicos de bancos de dados orientados a objetos, e as diversas formas de informação incompleta nesses bancos. As principais idéias e resultados apresentados aqui estão em [Zic90], [ZP92].

\subsubsection{Conceitos básicos}

Em bancos de dados orientados a objetos, a informação é organizada como objetos, que incorporam os dados e seu comportamento. A manipulação dos objetos é feita através de métodos, que são procedimentos associados aos objetos. Normalmente os dados incorporados em um objeto são uma tupla ou um conjunto de objetos.

Os objetos são organizados em classes, que descrevem suas estruturas e comportamento. A parte estrutural de uma classe é a definição dos tipos de dados que compóem os objetos, e a parte de comportamentos é um conjunto de métodos que manipulam estes objetos.

As classes são relacionadas umas com outras através do conceito de herança. A herança é um mecanismo que permite definir classes de forma construtiva, detalhando a definição de uma classe em função de outras. Normalmente, na definição de uma classe é necessário conhecer completamente a estrutura da mesma. No entanto, no momento da definição da classe, (isto é, no projeto do banco de dados) muitas vezes não possuímos a informação precisa a respeito do comportamento da mesma.

É importante, então, ter mecanismos para definir classes e objetos mesmo quando a informação a respeito dos mesmos não é totalmente especificada, especialmente na fase de projeto do banco. Destacamos então dois tipos de informação incompleta em bancos de dados orientados a objetos:

- Esquemas incompletos. Este tipo diz respeito à possibilidade de definir classes onde a estrutura e métodos de manipulação são parcialmente (ou totalmente) não especificados. 
- Objetos incompletos. Este tipo diz respeito à possibilidade de expressar valores desconhecidos para atributos do objeto

\subsubsection{Esquemas incompletos}

Classes com esquemas incompletos são denominadas classes genéricas [Zic90]. Nas classes genéricas, não especificamos os atributos que nela constam, apenas citamos sua existência. Isto não impede, no entanto, a sua utilização na definição de outras classes, como ilustra o exemplo a seguir:

\section{Class Professor \\ attributes Nome: string, \\ Depto: string.}

$\begin{array}{cc}\begin{array}{c}\text { Class Aluno } \\ \text { generic; }\end{array} & \text { Class AlunoPos inherits Aluno } \\ \text { generic; }\end{array}$

A classe Aluno, apesar de não estar ainda definida, pode ser utilizada na definição da classe AlunoPos.

Associamos a cada objeto um identificador e valores. Por exemplo, pode-se inserir na classe Professor um objeto identificado por Silva, com atributos Nome= "Carlos Silva" e Depto="MAC". Os objetos de uma classe genérica não têm valor definido. Por exemplo, não há valor algum associado aos objetos das classes Aluno e AlunoPos.

A principal razão para se criar uma classe genérica é permitir a definição de outras classes que dependam desta e não atrasar a definição do banco de dados como um todo. No entanto, futuramente as classes genéricas serão definidas. Nesse caso, todos os objetos já definidos na classe genérica assumem valores nulos (possivelmente padrão) nos atributos recém especificados.

\subsubsection{Objetos incompletos}

Segundo [Zic90], existem dois tipos de objetos incompletos:

- objetos pertencentes a classes genéricas: nesse caso, o objeto possui apenas um identificador, não possuindo nenhum valor. Associamos aos valores desse objeto o valor nulo $n_{I}$. 
- objetos pertencentes a classes cuja estrutura é definida, mas onde alguns dos atributos dessa classe não foram especificados, e portanto são desconhecidos.

No exemplo da seção anterior, os objetos das classes Aluno e AlunoPos pertencem à primeira categoria. Um objeto da classe Professor onde o atributo Depto é desconhecido pertence à segunda categoria de objetos incompletos.

Como abordagem inicial, podemos atribuir aos atributos desconhecidos do segundo tipo de objetos incompletos o valor nulo aplicável $n_{A}$. Para tornar o banco de dados mais representativo, pode-se atribuir qualquer um dos valores nulos já vistos, dependendo da situação, ou seja, o valor nulo sem informação $n_{S}$, os valores nulo universal ou padrão (quando se adota a OWA) ou o valor nulo aberto $n_{\Omega}$. Uma vez que os atributos das classes podem ser conjuntos, o valor nulo correspondente ao conjunto vazio também pode ser utilizado.

$\mathrm{Na}$ verdade, o tratamento de valores nulos em bancos de dados orientados a objetos é análogo ao de bancos de dados não normalizados interpretados sob a CWA. No entanto, quando se permite o uso de valores nulos em bancos de dados orientados a objetos, a definição dos métodos que utilizam estes objetos incompletos deve ser feita com cuidado. Cada vez que o projetista define um novo método, deve ter em mente a possibilidade de que o objeto em questão seja incompleto, e que possivelmente deva ter um tratamento diferenciado. 


\section{Capítulo 10}

\section{Proposta de implementação}

A seguir apresentamos um pequeno exercício de implementação de um sistema para gerenciamento de bases de dados (SGBD) com valores vazios. Nossa proposta segue o modelo de [Codd79]. As relações com valores vazios de Codd [Codd79], para a maioria das aplicações práticas, já são suficientes. Também por ser o modelo relacional o que foi usado no maior número de SGBD comerciais atuais, optamos por adotá-lo em nossa sugestão de implementação.

Adotando este modelo de representação, resta definir quais tipos de valores vazios utilizar e como tratá-los. A escolha deve levar em consideração o poder de representação e a complexidade computacional da solução. Optamos por utilizar, em nosso exercício, os valores vazios inaplicável $\left(n_{I}\right)$ e sem informação $\left(n_{S}\right)$.

A justificativa dessa escolha é bastante simples:

- O valor vazio $n_{S}$ é de fato um valor vazio, pois não há nenhuma informação sobre sua aplicabilidade. Este valor é o que aparece com mais frequência em aplicações práticas, como, por exemplo, na expansão do esquema de uma relação. Isto justifica a necessidade de implementação do mesmo.

- O valor vazio $n_{I}$ não traduz nenhum tipo de incerteza, é uma informação conhecida relativamente comum que se deseja representar. Por este motivo também deve ser implementado.

A não utilização do valor vazio $n_{A}$, no sentido em que não será feita a distinção entre o valor vazio sabidamente aplicável $\left(n_{A}\right)$ e o valor vazio que não se sabe sobre sua aplicabilidade $\left(n_{S}\right)$ é simplesmente para garantir a simplicidade da implementação. Nesse ponto concordamos com Zaniolo [Zan84], quando afirma que a informação extra obtida utilizando este valor vazio não justifica a complexidade que o mesmo introduz. 
A quantidade de informação representada pelos valores vazios escolhidos é esquematizada abaixo:

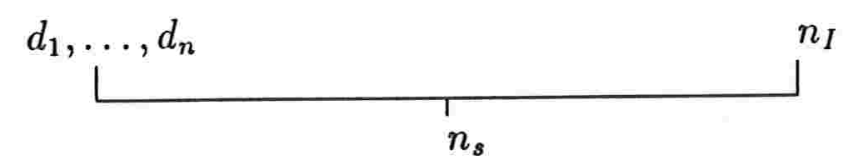

onde $d_{1}, \ldots, d_{n}$ são os valores não vazios dos domínios das relações. Pela figura notase que o valor vazio $n_{I}$ tem a mesma quantidade de informação que um elemento qualquer do domínio. Esta é mais uma razão que justifica sua inclusão em nossa implementação.

Definidos o modelo de representação e os tipos de valores vazios utilizados, partimos para as seguintes etapas do projeto:

- considerações teóricas.

- forma de implementação.

- definição de uma linguagem de acesso.

Essas definições são feitas a partir de conceitos já existentes. Sugerimos adotar a proposta de implementação da estrutura de dados de Setzer [Set89], e utilizamos a linguagem SQL como base para definição da linguagem de acesso.

\subsection{Considerações Teóricas}

Nesta seção apresentamos o tratamento teórico que adotamos para os valores vazios utilizados. Muitas das idéias aqui são inspiradas no trabalho de Zaniolo [Zan84]. No entanto, como decidimos pela utilização de dois tipos de valores vazios (Zaniolo só usa o vazio $n_{S}$ ), temos que propor algumas adaptações.

\subsubsection{Expressões de Comparação}

Ao adotar dois tipos de valores vazios, as expressões de comparação, bem como as expressões booleanas, utilizariam uma lógica de 4 valores: V, F, P, I. No entanto, resolvemos adotar as seguintes simplificações: 
- o valor vazio inaplicável $\left(n_{I}\right)$ não satisfaz nenhum tipo de comparação (a menos da igualdade entre valores inaplicáveis).

- o valor vazio sem informação satisfaz $\left(n_{S}\right)$ todos os tipos de comparação.

A justificativa para esta última é que, com a representação explícita do valor vazio $n_{I}$, há maior probabilidade de o valor vazio $n_{S}$ representar, na verdade, o valor vazio aplicável $n_{A}$. Comparações envolvendo $n_{A}$ não devem ser falsas, pois este valor representa um elemento qualquer no domínio, que pode satisfazer a condição especificada. Assim, assumimos Verdadeiras as expressões que podem envolver comparações com o valor vazio $n_{A}$, e consequentemente, o valor vazio $n_{S}$.

Dessa forma, estamos "disfarçando" a lógica multivalorada que existe por trás dos valores vazios. No entanto, é necessário fornecer ao usuário ferramentas para distinguir valores vazios de valores do domínio, quando necessário. Criamos então duas funçôes booleanas:

- $\operatorname{Vazio}(\mathrm{X})$ : Retorna $\mathrm{V}$ se o valor do atributo X é vazio (isto é, corresponde ao valor vazio $\left.n_{S}\right)$.

- Inaplicável(X): Retorma V se o valor do atributo X é inaplicável (isto é, corresponde ao valor vazio $\left.n_{I}\right)$.

As tabelas seguintes ilustram o resultado de expressôes de comparação (onde $d$ representa um valor não vazio):

\begin{tabular}{|c|c|}
\hline Termo & Valor \\
\hline$d>n_{S}$ & $\mathrm{~V}$ \\
$n_{S}>n_{S}$ & $\mathrm{~V}$ \\
$n_{S}>n_{I}$ & $\mathrm{~F}$ \\
$d>n_{I}$ & $\mathrm{~F}$ \\
$n_{I}>n_{I}$ & $\mathrm{~F}$ \\
\hline
\end{tabular}

\begin{tabular}{|c|c|}
\hline Termo & Valor \\
\hline$d=n_{S}$ & $\mathrm{~V}$ \\
$n_{S}=n_{S}$ & $\mathrm{~V}$ \\
$n_{S}=n_{I}$ & $\mathbf{F}$ \\
$d=n_{I}$ & $\mathbf{F}$ \\
$n_{I}=n_{I}$ & $\mathrm{~V}$ \\
\hline
\end{tabular}

Com estas simplificações, transferimos para o usuário a complexidade do tratamento de valores vazios. Como não utilizamos nenhum valor lógico além dos tradicionais $\mathrm{V}$ e F, a álgebra booleana não é alterada. No entanto, as expressões de comparação avaliam para $\mathbf{V}$ expressões, que, na verdade, deveriam ser $\mathbf{P}$. O usuário tem, agora, a responsabilidade de especificar se deseja obter resultados que correspondem somente ao valor lógico V. Por exemplo, considere a consulta: 


\section{"Listar funcionários com salário $\geq 3000$ "}

Quando não se deseja, no resultado da consulta, tuplas onde o salário é desconhecido, a consulta deve ser escrita na forma: "Listar funcionários com salário $\geq 3000$ e salário não é vazio", ou ampliando a sintaxe da linguagem, "Listar os funcionários que com certeza têm salário $\geq 3000$ ".

Outras expressões a serem analisada são as funções de agregação, que envolvem várias tuplas, como Média, Soma, Mínimo, Máximo. Nesse caso, o cálculo deve ser feito apenas sobre os campos não vazios, ou seja, diferentes de $n_{I}$ e $n_{S}$. Para o cálculo destas expressões, tuplas onde o atributo considerado é vazio são tratadas como se não existissem. A única exceção é a função Contador de tuplas que satisfazem uma dada expressão. Neste caso, o tratamento é semelhante ao de consultas de tuplas que satisfazem a expressão.

\subsection{2 Álgebra Relacional}

A definição dos 5 operadores básicos é suficiente para a caracterização completa da álgebra. Basicamente, os operadores não sofrem grande alteração, a menos do operador de seleção, que utiliza os conceitos apresentados para avaliação de expressões de comparação. Para os demais, a definição é idêntica àquela sem valores vazios. $\mathrm{Ou}$ seja, interpretamos $n_{S}$ e $n_{I}$ como valores quaisquer do domínio.

- $\Pi_{X}(\mathrm{r})=\{\mathrm{r}[\mathrm{X}]\}$

- $r \cup s=\{t \mid t \in r$ ou $t \in s\}$

- $\mathrm{r}-\mathrm{s}=\{\mathrm{t} \mid \mathrm{t} \in \mathrm{r}$ e $\mathrm{t} \notin \mathrm{s}\}$

- $\mathrm{r} \times \mathrm{s}=\left\{t_{1} \& t_{2} \mid t_{1} \in \mathrm{r}\right.$ e $\left.t_{2} \in \mathrm{s}\right\}$ onde " $\&$ " representa a concatenação de tuplas.

- $\sigma_{\Theta}(r)=\{t \in r \mid t$ satisfaz a expressão $\Theta\}$, onde a "satisfabilidade" da expressão é interpretada como descrita na seção anterior.

A operação de junção, apesar de não integrar o grupo das cinco básicas, merece um comentário especial. O resultado de uma operação de junção, segundo a avaliação de expressões adotada, é uma relação que engloba, além das tuplas que normalmente seriam incluídas em uma definição sem considerar valores vazios, as possíveis combinações dos valores vazios. Por exemplo, considere as relações a seguir: 


\begin{tabular}{|c|c|}
\hline$A$ & $B$ \\
\hline \hline$a_{1}$ & $b_{1}$ \\
$a_{2}$ & $b_{2}$ \\
$a_{2}$ & $n_{S}$ \\
$a_{2}$ & $n_{I}$ \\
\hline
\end{tabular}$\quad$\begin{tabular}{|c|c|}
\hline$B$ & $C$ \\
\hline \hline$b_{1}$ & $c_{1}$ \\
$n_{S}$ & $c_{2}$ \\
$b_{2}$ & $n_{S}$ \\
$b_{3}$ & $c_{3}$ \\
\hline
\end{tabular}$\quad$\begin{tabular}{|c|c|}
$B^{\prime}$ & $C$ \\
\hline \hline$b_{1}$ & $c_{1}$ \\
$n_{S}$ & $c_{2}$ \\
$b_{2}$ & $n_{S}$ \\
$b_{3}$ & $c_{3}$ \\
\hline
\end{tabular}

\begin{tabular}{|c|c|c|}
\multicolumn{3}{c}{$r \bowtie s$} \\
\hline$A$ & $B$ & $C$ \\
\hline \hline$a_{1}$ & $b_{1}$ & $c_{1}$ \\
$a_{1}$ & $b_{1}$ & $c_{2}$ \\
$a_{2}$ & $b_{2}$ & $c_{2}$ \\
$a_{2}$ & $b_{2}$ & $n_{S}$ \\
$a_{2}$ & $b_{1}$ & $c_{1}$ \\
$a_{2}$ & $n_{S}$ & $c_{2}$ \\
$a_{2}$ & $b_{3}$ & $c_{3}$ \\
\hline
\end{tabular}

\begin{tabular}{|c|c|c|c|}
\multicolumn{4}{c}{$r \bowtie_{B=B^{\prime}} s^{\prime}$} \\
\hline$A$ & $B$ & $B^{\prime}$ & $C$ \\
\hline \hline$a_{1}$ & $b_{1}$ & $b_{1}$ & $c_{1}$ \\
$a_{1}$ & $b_{1}$ & $n_{S}$ & $c_{2}$ \\
$a_{2}$ & $b_{2}$ & $n_{S}$ & $c_{2}$ \\
$a_{2}$ & $b_{2}$ & $b_{2}$ & $n_{S}$ \\
$a_{2}$ & $n_{S}$ & $b_{1}$ & $c_{1}$ \\
$a_{2}$ & $n_{S}$ & $n_{S}$ & $c_{2}$ \\
$a_{2}$ & $n_{S}$ & $b_{2}$ & $n_{S}$ \\
$a_{2}$ & $n_{S}$ & $b_{3}$ & $c_{3}$ \\
\hline
\end{tabular}

Formalmente, sejam $r(R)$ e $s(S)$ duas relações. A junçẫo natural de $r$ e $s$, denotado por $r \bowtie s$, é definida da forma:

$r \bowtie s=\Pi_{R \cup S}\left(r \bowtie_{\Theta} s\right)$, onde $\Theta=\left(\left(r\left[A_{1}\right]=s\left[A_{1}\right] \vee r\left[A_{1}\right]=n_{S} \vee s\left[A_{1}\right]=\right.\right.$ $\left.\left.n_{S}\right) \wedge \ldots \wedge\left(r\left[A_{n}\right]=s\left[A_{n}\right] \vee r\left[A_{n}\right]=n_{S} \vee s\left[A_{n}\right]=n_{S}\right)\right)$, e $R \cap S=\left\{A_{1}, \ldots, A_{n}\right\}$. Para $\mathrm{i}=1, \ldots, \mathrm{n}$, se $r\left[A_{i}\right]=n_{S}$ então, no cálculo da projeção toma-se o valor do atributo $s\left[A_{i}\right]$ para compor $r \bowtie s\left[A_{i}\right]$, e se $s\left[A_{i}\right]=n_{S}$ então, no cálculo da projeção toma-se o valor do atributo $r\left[A_{i}\right]$ para compor $r \bowtie s\left[A_{i}\right]$.

Basicamente, esta definição é bastante similar à definição de Codd [Codd79] de "junção possível". Isto se deve ao fato de considerarmos as expressões originalmente "Possíveis" como "Verdadeiras". Note que, na junção natural, quando os valores dos atributos não são iguais (por exemplo, na comparação de $n_{S}$ e $b_{1}$ ), suprimimos sempre o valor menos informativo, ou seja, o valor vazio.

Para a definição desses operadores, não utilizamos nenhum tipo de tratamento especial para valores vazios, como o princípio da substituição de vazio [Codd79] ou as relações estendidas [Zan84]. O principal problema decorrente dessa simplificação é a eventual presença de tuplas redundantes, como ilustra o exemplo a seguir: 


\begin{tabular}{|l|l|}
\hline \multicolumn{2}{c}{$r$} \\
\hline Aluno & Curso \\
\hline Alves & Cálculo \\
Bastos & $n_{S}$ \\
\hline
\end{tabular}

\begin{tabular}{|l|l|}
\hline \multicolumn{2}{c}{$s$} \\
\hline Aluno & Curso \\
\hline Bastos & Geometria \\
Alves & Cálculo \\
Cintra & Geometria \\
\hline
\end{tabular}

\begin{tabular}{|l|l|}
\multicolumn{2}{c}{$r \cup s$} \\
\hline Aluno & Curso \\
\hline Alves & Cálculo \\
Bastos & Geometria \\
Bastos & $n_{S}$ \\
Cintra & Geometria \\
\hline
\end{tabular}

No exemplo, é possível que a tupla (Bastos, $n_{S}$ ) seja redundante, isto é, Bastos seria na verdade um estudante de Geometria, e este fato já está representado na relação. No entanto, isto pode não ser verdade, ou seja, a informação (Bastos, $n_{S}$ ) pode representar que Bastos está matriculado em outro curso que não Geometria. Assim, não é tão prejudicial a presença dessas tuplas "redundantes" no resultado da operação. Adotamos esta definição dos operadores relacionais para simplificação. No entanto, nada impede que se utilize o tratamento descrito em [Zan84] para eliminação de redundâncias.

\subsubsection{Atualização}

Adotamos os operadores usuais de atualização: Inserção, Alteração e Remoção. Como apresentado no Capítulo 7, a representação utilizando relações com valores vazios não é suficiente para expressar todos os operadores de atualização em bancos de dados com valores vazios. No entanto, a complexidade computacional da representação ideal, as C-tabelas, é bem maior, justificando a opção pelo modelo mais simples.

\subsection{Forma de implementação}

Para implementar os dois tipos de valores vazios eleitos, faremos uma pequena adaptação ao modelo proposto por Setzer [Set89] para implementação da estrutura de dados de um banco de dados. Este modelo permite inclusive a representação de relações não normalizadas. Assim, nossa sugestão de implementação também terá recursos para representar os dois tipos de valores vazios em relações não normalizadas.

A solução original é baseada em registros de tamanho variável, e implementa ainda compressão de dados eliminando zeros à esquerda de campos numéricos e brancos à direita de campos alfanuméricos. Para nós, a característica mais importante dessa solução, que será vital para a representação dos valores vazios, é a supressão dos campos vazios. 
A estrutura de dados utilizados é baseada na localização dos dados de uma coluna pela ordem de sua posição, no registro correspondente, da esquerda para a direita. $\mathrm{Na}$ solução original, para implementar essa localização pela ordem, coloca-se na frente (esquerda) do campo um "byte" com a contagem de "bytes" do campo seguinte. Optamos por colocar na frente do campo um "byte" de controle com um apontador relativo para o próximo campo. Dessa forma, o percurso de campos é mais rápido, pois não é necessário somar 1 a cada vez. Por exemplo, considere a relação ExameReq a seguir, que representa a requisição de exames laboratoriais para pacientes:

\begin{tabular}{|l|l|l|l|l|}
\multicolumn{9}{c}{ ExameReq } \\
\hline Paciente & Exame & Requisição & EntregaMat & Resultado \\
\hline \hline Alves & Urina I & $01 / 09 / 93$ & $n_{S}$ & $n_{S}$ \\
Bastos & Urina I & $10 / 08 / 93$ & $12 / 08 / 93$ & $14 / 08 / 93$ \\
Cintra & Ultrassonografia & $15 / 08 / 93$ & $n_{I}$ & $n_{S}$ \\
\hline
\end{tabular}

O atributo Requisição representa a data de requisição do exame; o atributo EntregaMat indica a data de entrega do material para exame e o atributo Resultado indica a data em que o resultado do exame ficou pronto.

A implementação da segunda linha dessa relação ficaria:

\begin{tabular}{|l|l|l|l|l|l|l|l|l|l|}
7 & Bastos & 8 & Urina I & 7 & 930810 & 7 & 930812 & 7 & 930814 \\
\hline
\end{tabular}

Como em [Set89], o "bit" mais à esquerda do "byte" de controle indica com "0" que não há supressão da(s) próxima(s) coluna(s); um "1" indica supressão dos números de campos representados pelos 7 "bits" mais à direita. Dessa forma, pode-se indicar até 128 campos vazios (0 a 127). Se for necessário mais do que isso, repete-se novamente o "byte" de contagem com o "bit" mais à esquerda novamente com o valor "1". Em nosso modelo, a supressão é a representação interna do valor vazio $n_{S}$. Optamos por este valor vazio para ser suprimido pois acreditamos que é mais comum que o valor vazio $n_{I}$. A representação interna da primeira linha da mesma relação ExameReq seria:

\begin{tabular}{|l|l|l|l|l|l|l|}
6 & Alves & 8 & Urina I & 7 & 930901 & 130 \\
\hline
\end{tabular}

onde 130 é o valor decimal do byte

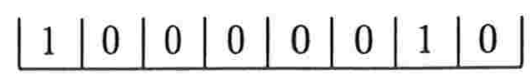


indicando que existem 2 campos vazios não informados, representando os valores vazio $n_{S}$ nos atributos EntregaMat e Resultado.

A representação interna do valor vazio $n_{I}$ é feita utilizando-se um campo de tamanho 0 , ou seja, o campo contém o valor " 1 ", indicando que o próximo campo está um "byte" à frente. Dessa forma, não se indica que o campo é suprimido, mas que não há valor a ser representado. Este método é coerente com a semântica do valor vazio $n_{I}$. Ainda no exemplo de requisição de exames, a terceira linha da relação ExameReq seria representada da forma

\begin{tabular}{|l|l|l|l|l|l|l|l|}
7 & Cintra & 17 & Ultrassonografia & 7 & 930815 & 1 & 129 \\
\hline
\end{tabular}

onde 1 e 129 representam, respectivamente:

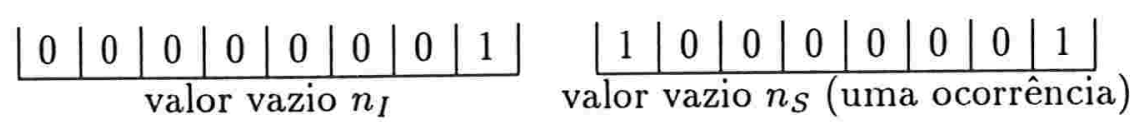

Nessa representação, não há necessidade de alterar o modelo original de implementação, apenas atribuímos um significado a certas configurações. Assim, além de mantermos a mesma estrutura de dados inicial, não precisamos desperdiçar espaço para armazenar as marcas correspondentes aos dois valores vazios distintos.

Em [Set89], temos mais detalhes sobre o modelo utilizado. Nos exemplos apresentados, os campos têm tamanho pequeno, mas o modelo permite a representação de campos de tamanho ilimitado, encadeando-se um número variável de "bytes" de controle em campos não vazios. É detalhada também a forma de representação de relações não normalizadas.

\subsection{Indexação}

Os gerenciadores de bancos de dados permitem a definição de índices para determinados atributos das relações, de modo a melhorar o tempo de acesso às tuplas, que normalmente são interrogadas por estes atributos. Por exemplo, em uma relação Funcionários(Num-Func, Nome-Func, Salário), é razoável imaginar que serão submetidas diversas consultas fornecendo o nome do funcionário, e portanto deveria existir uma estrutura de indexação sobre o atributo Nome-Func.

As estruturas de indexação (B-árvores, por exemplo) poderiam permitir também o uso dos valores vazios. Dessa forma, uma consulta do tipo "Liste os funcionários cujo 
salário é desconhecido" seria muito mais rápida se o atributo Salário fosse indexado e os valores vazios pudessem ser colocados na estrutura do índice.

Para permitir o uso de valores vazios nessas estruturas, seria necessário apenas definir dois valores especiais (correspondentes aos valores vazios $n_{S}$ e $n_{I}$ ). Dessa forma, estes valores especiais apontariam para as tuplas que contém valores vazios nos atributos indexados.

A maioria dos SGBD comerciais (pelo menos que temos conhecimento), no entanto, não permite este tipo de implementação. Conversamos com alguns usuários de alguns SGBD (ZIM, da Sterling, DMS-II, da Unisys e ADABAS, da Software A.G.) e percebemos que não é possível definir índices para valores vazios. Dessa forma, as consultas envolvendo valores vazios serão sempre ineficientes. É uma pena, pois do ponto de vista teórico não haveria praticamente nenhuma dificuldade adicional para utilizar valores vazios também nos índices.

\subsection{Linguagem de Acesso}

A linguagem sugerida é uma pequena variação do SQL que incorpora o tratamento dos valores vazios especificados. Devemos ter funções correspondentes ao Vazio e Inaplicável definidos na seção anterior. Por motivos estéticos, uma vez que as cláusulas SQL são todas escritas em inglês usaremos a sintaxe:

- $X$ is unknown : função $\operatorname{Vazio}(X)$

- X is nonapplicable : função Inaplicável(X)

O valor null, já existente na sintaxe de SQL, pode ser utilizado para denotar os dois tipos de valores vazios. Dessa forma, $\mathrm{X}$ is null é equivalente a $\mathrm{X}$ is unknown or $\mathrm{X}$ is nonapplicable em nossa linguagem.

Como já citado, as consultas retornavam as tuplas que satisfazem ou que podem satisfazer a expressão indicada. Para selecionar apenas as tuplas que satisfazem com certeza determinadas condições, deve-se utilizar a função unknown. Para conforto dos usuários, no entanto, podemos definir um operador selectC ("select with certainty"), idêntico ao operador select, que retorna apenas as tuplas que satisfazem com certeza as condições especificadas. Por exemplo, a consulta 


\author{
select Nome-Func \\ from Funcionarios \\ where Salario-Func $\geq 3000$ and \\ Salario-Func is not unknown
}

seria escrita simplesmente da forma

$\begin{array}{ll}\text { selectC } & \text { Nome-Func } \\ \text { from } & \text { Funcionarios } \\ \text { where } & \text { Salario-Func } \geq 3000\end{array}$

Não é necessário criar um operador semelhante para o tratamento do valor vazio inaplicável pois o resultado de operações envolvendo este valor vazio é sempre falso. As consultas que se pode fazer a campos inaplicáveis são escritas de maneira confortável usando os recursos já existentes. Por exemplo, suponha que se deseja listar os funcionários que não recebem salários (por exemplos, os proprietários da empresa). A consulta fica da forma:

\title{
select Nome-Func \\ from Funcionarios \\ where Salario-Func is nonapplicable
}

$\mathrm{Na}$ sintaxe dos operadores de atualização, é necessário prover meios para que o usuário possa informar que um atributo é inaplicável ou desconhecido para aquela tupla. Podemos definir as constantes nonapplicable e unknown, que podem ser utilizados nos comandos insert e update, respectivamente nas cláusulas values e set. Por exemplo, para criar uma tupla na relação Funcionarios(Nome-Func,SalarioFunc) onde não se tem informação sobre o salário, temos:

$$
\begin{array}{ll}
\text { insert into } & \begin{array}{l}
\text { Funcionarios } \\
\text { values }
\end{array} \\
\text { ("Morais", unknown) }
\end{array}
$$

Para alterar o salário desse funcionário para inaplicável, podemos escrever:

$$
\begin{array}{ll}
\text { Update } & \text { Funcionarios } \\
\text { set } & \text { Salario-Func = nonapplicable } \\
\text { where } & \text { Nome-Func = "Morais" }
\end{array}
$$


A linguagem de definição do banco de dados deve ainda, fornecer subsídios para definir que um determinado atributo em uma relação não possa assumir valores vazios. Isto seria interessante, por exemplo, para os atributos que fazem parte da chave primária da relação.

Acreditamos que estes recursos extras para tratamento de valores vazios são suficientes para resolver a maioria dos problemas práticos. A seguir apresentamos alguns exemplos de aplicações com as quais tivemos contato em nossa experiência profissional onde aparece o problema do valor vazio, e como seria possível resolvê-lo utilizando o método apresentado.

\subsection{Exemplos}

\subsubsection{Administração de Recursos Humanos}

Considere as seguintes relações, parte de um sistema para administração de recursos humanos, como foi implementado na prática:

Funcionários
\begin{tabular}{|l|l|}
\hline Num & Nome-Func \\
\hline \hline 19 & Bastos \\
27 & Cabral \\
35 & Silva \\
43 & Soares \\
\hline
\end{tabular}

Designação

\begin{tabular}{|l|l|l|}
\hline Num & Cargo & Num-Subst \\
\hline \hline 19 & Gerente & 0 \\
27 & Diretor & 0 \\
35 & Gerente & 19 \\
\hline
\end{tabular}

A relação Designação indica o cargo de chefia ocupado por um funcionário. O atributo Num-Subst indica que o funcionário não é efetivo no cargo, está apenas substituindo outro que, por exemplo, está em férias. Em um SGBD que não permite a representação de valores vazios, utilizamos 0 para para indicar que o funcionário não está substituindo ninguém. Com valores vazios, porém, este campo deveria ser preenchido com um número de funcionário ou com o valor vazio $n_{I}$, indicando, que neste caso, o atributo não se aplica.

\subsubsection{Organograma}

A relação a seguir representa os setores de uma empresa em sua organização hierárquica: 


\begin{tabular}{|l|l|}
\multicolumn{2}{c}{ Setores } \\
\hline Setor & Superior \\
\hline \hline Presidência & \\
Diretoria Administrativa & Presidência \\
Diretoria de R.H. & Presidência \\
Diretoria de Produção & Presidência \\
\hline
\end{tabular}

O setor hierarquicamente mais alto não possui superior, e este campo foi então definido como branco. A representação utilizando valores vazios permite um tratamento correto da situação, colocando no campo em questão o valor vazio $n_{I}$.

\subsubsection{Controle Acadêmico}

Considere a relação Matrícula a seguir, representando os dados de alunos matriculados em cursos:

\begin{tabular}{|l|l|l|l|l|}
\multicolumn{5}{|c}{ Matrícula } \\
\hline Aluno & Discip & AnoSem & Freq & Nota \\
\hline \hline Bastos & Álgebra & $93 / 2$ & 0 & 0 \\
Cintra & Geometria & $92 / 2$ & 100 & 9,5 \\
Dutra & Cálculo & $92 / 2$ & 0 & 0 \\
\hline
\end{tabular}

A única forma de saber se a frequência e notas de um aluno já foram lançados é através do atributo AnoSem (ano e semestre de matrícula). Quando este corresponde ao ano/semestre atual, ainda não findo, indica que o curso ainda está em andamento, e portanto a frequência e nota ainda não foram lançados. Este tratamento causa muita confusão, pois um usuário menos atento pode pensar que o aluno foi reprovado na disciplina, quando na verdade ele ainda nem foi avaliado.

Com a utilização de valores vazios, os campos frequência e nota contêm o valor $n_{S}$ antes do lançamento das notas, e no histórico escolar pode ser impresso um texto como "Vazio", "Desconhecido", ou deixado em branco, indicando que o campo ainda não foi informado, eliminando a confusão.

\subsubsection{Requisições de Exames}

Considere a relação ExameReq, da seção 9.2. O atributo EntregaMat, em certos casos, não se aplica, como no exemplo do exame de ultrassonografia. Nesses casos, 
isto pode ser representado com o uso da valor vazio $n_{I}$. Por outro lado, os atributos EntregaMat e Resultado são informados somente quando os eventos correpondentes ocorrem. Antes disso, a representação correta seria feita utilizando o valor vazio $n_{S}$. 


\section{Capítulo 11}

\section{Conclusões}

O tratamento de informações incompletas em bancos de dados é um problema que ainda merece ser objeto de muito estudo. As abordagens algébrica e lógica fornecem subsídios importantes para a compreensão teórica do problema, mas muitas questões ainda permanecem em aberto. Como a imperfeição dos dados assume as mais variadas formas, torna-se difícil encontrar uma representação que possa, ao mesmo tempo, ser fiel à realidade e com uma complexidade computacional razoável.

Em vários pontos desse texto comentamos que é muito difícil compreender a intuição por trás das definições matemáticas (é o caso, por exemplo, dos operadores da lógica de 7 valores). Não existe sequer um consenso sobre qual a melhor abordagem teórica a ser adotada.

O problema da representação fiel da realidade é bastante complexo. No entanto, por aparecer frequentemente na prática, tem sido exaustivamente estudado, mas, como comenta [ZP92], o custo para se obter resultados precisos nesta área é alto.

A sugestão apresentada de implementação e tratamento de valores vazios pretende ser um passo a mais na tentativa de representar com mais fidelidade os fatos no mundo real. A estrutura adotada não é a que possui o maior poder de representação dentre as apresentadas, mas pensamos que é a que possui a melhor relação entre custos e benefícios, pois resolve diversos problemas de aplicações práticas, e praticamente não tem nenhum custo computacional adicional.

Através desse trabalho, vimos que existem vários pontos que podem ser objetos de pesquisa futura, como por exemplo, a especificação completa de uma linguagem para implementação, a otimização de consultas com valores vazios, o estudo mais detalhado para a implementação de índices e a realização de testes práticos para eficiência. Outro aspecto interessante é o estudo da facilidade de compreensão do uso 
dos diversos tipos de valores nulos junto aos usuários finais.

A maior contribuição deste trabalho é o fato de ser uma resenha sobre uma área recente e ainda em fase de pesquisa. Encontramos apenas outra resenha sobre o assunto [ZP92] que, por abordar aspectos mais diversos de incerteza em bancos de dados, não se aprofunda tanto na descrição dos valores vazios. É importante destacar que a bibliografia consultada foi detalhadamente estudada, de forma a conseguirmos uma bibliografia comentada que por si só já pode ser útil para base de pesquisas na área.

Nossa resenha permite o uso para fins didáticos em disciplinas de pós-graduação, além de poder ser usada como base inicial para pesquisas na área. Apesar do volume relativamente pequeno, consideramos a proposta de implementação importante, pois os SGBD, quando fazem o tratamento de valores vazios, o fazem parcialmente, não fazendo distinção entre tipos e não permitindo a indexação. 


\section{Bibliografia Comentada}

[AG85] S.Abiteoul and G.Grahne. Update semantics for incomplete databases. In Proc. 11o. International Conference on Very Large Databases, Stockholm, 1985, pp.1-12.

Discorre sobre tipos possíveis de atualizações em bancos de dados incompletos, introduzindo novas operações de atualização: integração, sujeição (negativa) e adição, além de generalizar os conceitos de inserção, remoção e alteração. O significado de cada operador é definido, e são apresentados resultados sobre tipos de representação que suportam esses operadores.

[ANS75] ANSI/X3/SPARC. Study group on Data Base Management Systems Interim Report. SIGMOD FDT Bull., 7(2), 1975.

Lista 13 tipos diferentes de valores vazios.

[Bis83] J.Biskup. A foundation of Codd's relational and maybe-operations. ACM Transactions on Database Systems, 8(4), December 1983, pp.608-636.

Apresenta-se uma semântica para bancos de dados com valores vazios, com base nos modelos (da lógica de primeira ordem) representados por uma tupla com valores vazios, isto é, (a,b,c,w,e) é uma representação para $\exists x(\mathrm{a}, \mathrm{b}, \mathrm{c}, x, \mathrm{e})$. Nesse contexto, $\omega$ pode ser entendido como a constante de Skolem. São feitas considerações a respeito de fatos negativos, e é apresentada uma versão aplicável a bancos de dados com vazios da CWA que, basicamente, não permite assumir fatos negativos a respeito de uma tupla com valores vazios. Cria-se um sistema formal para representação de relações com vazios, e a álgebra relacional é estendida para este sistema. O conceito de redundância e quantidade de informação é definido sintaticamente, e são construídos operadores para remoção de redundância.

[CB80] M.A.Casanova and P.A.Bernstein. A formal system for reasoning about programs acessing a relational database. ACM Transactions on Programming Languages and Systems, 2(3), July 1980, pp.387-414. 
Introduz um sistema formal para demonstrar propriedades de programas que acessam bancos de dados relacionais, utilizando lógica dinâmica. Os problemas de preservação de consistência em atualizações são vistos sob esta ótica, permitindo demonstrar formalmente a preservação de integridade após a execução do programa.

[Cst87] J.M.Castilho. Especificações formais e sistemas de bancos de dados. Ed.prelim.Kapelusz, Buenos Aires, 1987.

Destaca o uso de formalismos para descrever bancos de dados, ou seja, o formalismo algébrico e lógico. Apresenta mais detalhadamente o formalismo lógico.

[Cla78] K.L.Clark. Negation as failure. In Logic and Databases, H.Gallaire and J.Minker, Eds., Plenum Press, New York, 1978, pp.293-322.

Mostra que a regra de negação por falha, isto é, a regra: "se não temos $\vdash$ p então temos $\vdash \neg$ p", é correta para bancos de dados. Coloca o banco de dados como um tipo especial de programa em lógica, e analisa a avaliação de consultas como prova de teoremas. Para provar fatos negativos, introduz a negação por falha finita, que utiliza, além da regra de negação por falha, o método da resolução. O método apresentado é correto, e completo em algumas situações particulares.

[Codd70] E.F.Codd. A relational model for large shared data banks. Commun. ACM 13(6), June, 1970, pp.377-387.

Introduz o modelo relacional para bancos de dados, e o conceito de relações normalizadas e não-normalizadas.

[Codd72] E.F.Codd. Further normalization of the database relational model. In Database Systems, Courant Computer Science Symposium 6, R.Rustin, Ed., PrenticeHall, Englewood Cliffs, 1971, pp.65-98.

Introduz os conceitos de dependência funcional, de Segunda e Terceira Formas Normais. Apresenta diversos exemplos que justificam este tipo de padronização, que elimina várias anomalias de atualização em bancos de dados.

[Codd79] E.F.Codd. Extending the database relational model to capture more meaning. ACM Transactions on Database Systems, 4(4), December 1979, pp.397-434.

Introduz um novo modelo, extensão do modelo relacional. Para tanto, sugere uma extensão da álgebra relacional, para incorporar o tratamento de valores vazios, introduzindo uma lógica de três valores (Verdadeiro, Falso, Desconhecido), que é usada também na extensão da álgebra relacional. São apresentados operadores específicos para bancos de dados com valores vazios: a união externa, a teta-junção externa, e a junção natural externa. 
[Codd86] E.F.Codd. Missing information (applicable and inapplicable) in relational databases. SIGMOD Record, 15(4), December 1986, pp.53-78.

Comenta aspectos gerais do tratamento de valores vazios (aplicáveis e inaplicáveis) em bancos de dados. Propóe a lógica de 3 valores para o tratamento dos dois tipos de vazios, e defende a tese de que os vazios devem ser entendidos semanticamente como marcas e não como valores especiais no domínio dos atributos. Sob esta ótica, os operadores de comparação retornam sempre "pode ser" para expressões que envolvem algum tipo de valor vazio; a ordenação é apenas simbólica, i.e, todos os símbolos I-marcas ou A-marcas vêm antes dos outros valores; o cálculo envolvendo valores vazios não é razoável; e, finalmente, as restrições de integridade e regras de normalização só devem ser avaliadas em tuplas completas (sem valores vazios).

[Codd87] E.F.Codd. More comentary on missing information in relational databases. SIGMOD Record, 16(1), March 1987, pp.42-50.

$\mathrm{O}$ objetivo do artigo é esclarecer pontos que possam ter ficados confusos no artigo anterior, dentre eles: 1)explica que, por questões de simplicidade, a integridade referencial leva em conta apenas chaves com um só atributo, os demais casos, quando necessário, podem ser incorporados em restrições de integridade geral; 2)defende a idéia de que a lógica de 4 valores (Falso, Verdadeiro, Possível, Inaplicável) pode e deve ser simplificada para 3 valores, para efeito de simplicidade de tratamento e compreensão; 3)salienta as diferenças entre operadores lógicos e funções escalares aplicadas a tuplas com marcas; 4)coloca algumas implementações necessárias para uma linguagem de acesso a bancos de dados com marcas.

[GMN84] H.Gallaire, J.Minker and J.Nicolas. Logic and Databases: A deductive approach. Computing Surveys, 16(2), June 1984, pp.153-185.

Apresenta o uso da lógica para o estudo de vários problemas na área de bancos de dados, tanto convencionais como dedutivos. É apresentada a visão de um banco de dados através da lógica, via conceitos semânticos (teoria de modelos) e sintáticos (teoria de prova). No campo de bancos de dados convencionais, mostra-se que os seguintes problemas encontram tratamento interessante em lógica: definição de linguagens de acesso, valores vazios, restrições de integridade, prova de consistência em transações, otimização de consultas e modelagem de bancos de dados. Na área de banco de dados dedutivos, a lógica faz parte da própria definição do banco, que basicamente é um bancos de dados convencional visto logicamente através de teoria de prova e dotado de regras específicas para dedução de fatos. Definem-se conceitos como a hipótese do mundo fechado (CWA) para tratar fatos negativos e a hipótese do mundo fechado generalizada (GCWA), para operacionalizar este tratamento. São apontadas as diferenças entre bancos com informação definida e 
indefinida (isto é, que contêm disjunções). Coloca-se também a semelhança com o campo de programação em lógica e questiona-se a possibilidade de se incorporar tratamento de funções.

[Ges90] G.H.Gessert. Four valued logic for relational database systems. SIGMOD Record, 19(1), March 1990, pp.29-35.

Introduz uma lógica de 4 valores para tratar o problema dos valores vazios : aplicável e falso (AF), aplicável e possível (AM), aplicável e verdadeiro (AT) e não aplicável (NA). O autor defende o uso dessa lógica, apesar de ser mais complicada que a lógica de 3 valores. Segundo ele, há casos em que se deseja informação precisa (especialmente se envolver os valores vazios inaplicáveis), o que não é possível na lógica de 3 valores. É apresentado também o uso dessa lógica em expressões de comparação em linguagens tipo SQL. É possível especificar-se, por exemplo, expressões do tipo "Status=Inaplicável".

[Ges91] G.H.Gessert. Handling missing data by using stored truth tables SIGMOD record, 20(3), September 1991, pp.30-42.

Propõe o uso de uma estrutura auxiliar para cada tabela no banco de dados, para armazenar os valores lógicos associados a cada um dos valores de cada uma das tuplas na tabela original. Assim, evitam-se problemas provenientes de se "misturar" valores (dados) com lógica - o que acontece quando se implementa o campo com um "byte" escondido para denotar a presença de valores vazios. Ganha-se em uniformidade de tratamento, e, dessa forma, pode-se, por exemplo, fazer cálculos sobre colunas que contenham valores vazios. A tabela lógica associada possui dados na lógica de 4-valores : NA, AF, AM e AT. Não é feita nenhuma consideração sobre a eficiência do método apresentado.

[Gra77] J.Grant. Null values in a relational database. Information Processing Letters, 6(5), October 1977, pp.156-157.

O autor aponta problemas na definição da lógica de 3 valores proposta por Codd. Basicamente, mostra que tautologias são avaliadas para o valor lógico Desconhecido, ao invés de Verdadeiro, como seria de se esperar. Propõe um outro método de avaliação de expressões, onde, para cada vazio, é feita a substituição por todos os elementos do domínio. Se em todas as substituições a expressão for avaliada Verdadeira, ou Falsa, a expressão é avaliada para esses valores. Senão, é avaliada para Desconhecido. É introduzido o conceito de vazio Inaplicável, que quando aparece numa expressão, torna a mesma falsa.

[Gra79] J.Grant. Partial values in a tabular model. Information Processing Letters, 9(2), August 1979, pp.97-99. 
O autor aponta a impossibilidade de se representar intervalos de valores em tabelas, que só permitem a representação de valores atômicos. É proposto então um modelo que permite a representação dos intervalos.

[HPRV 89] G.Hulin, A.Pirotte, D.Roelants and M.Vauclair. Logic and Databases. In From Modal Logic to Deductive Databases - Introducing a Logic Based Approach to Artificial Intelligence, A.Thayse, Ed., Vol.2, Wiley, 1989, pp.279-350.

Descreve a semântica de bancos de dados relacionais através da lógica de primeira ordem. Descreve também bancos de dados dedutivos, e comenta aspectos de informação incompleta em bancos de dados, entre eles o problema dos valores vazios.

[JS82] G.Jaeschke and H.J.Scheck. Remarks on the Algebra of Non-First Normal Form relations. In Proc. 1st ACM SIGACT/SIGMOD Symposium in Principles of Database Systems, Los Angeles, 1982, pp.124-138.

Introduz os operadores especiais para relações não normalizadas nest (que "normaliza" a relação em um nível) e unnest (que "não-normaliza a relação em um nível).

[IL84] T.Imielinski and W.Lipski. Incomplete information in relational databases. Journal of the ACM, 31(4), October 1984, pp.761-791.

São apresentados os conceitos de: 1)sistemas de representação, para comparar o tipo de informação que pode ser corretamente representada nos diversos modelos de bancos de dados. 2)V-tabelas, um modelo para representar bancos de dados com valores vazios onde se usam variáveis como na lógica de primeira ordem para representar os vazios. 3)C-tabelas, ou tabelas condicionais, extensões das V-tabelas onde para cada tupla existe uma coluna a mais para armazenar condições para que aquela tupla seja válida. Esses conceitos são trabalhados para comparar o poder de representação correta entre as tabelas de Codd com valores vazios, as V-tabelas e as C-tabelas.

[KS86] H.Korth and A.Silberschatz. Database system concepts. McGraw Hill, New York, 1986.

Apresenta diversos aspectos da teoria de bancos de dados, como os modelos de dados relacional, hierárquico e em rede. Aborda também conceitos de estrutura física de bancos de dados. Texto geral sobre bancos de dados. Consultado para descrever os conceitos iniciais apresentados no capítulo 2 .

[LP76] M.Lacroix and A.Pirotte. Generalized Joins. SIGMOD Record, 8(3), September 1976, pp.14-15. 
Analisa os operadores de junção estendidos para o tratamento com vazios, apresentados por Codd. Define generalização desses e apresenta novos operadores de junção não simétricos para bancos de dados com valores vazios.

[LS88] D.Laurent and N.Spyratos. Partition semantics for incomplete information in relational databases. SIGMOD Record, 17(3), pp.66-73, September 1988.

Introduz uma semântica de partições para bancos de dados com informação incompleta e apresenta um algoritmo para avaliação de consultas no banco. Basicamente, a idéia é, para um dado banco, calcular os possíveis modelos (que, no caso onde não há informação incompleta, é um só) e, para uma tupla qualquer:

- se a mesma está em todos os modelos, pode ser concluída com certeza a partir das informações do banco

- se a mesma está em algum dos modelos, pode ser concluída possível partir das informações do banco

- se a mesma não está em nenhum modelo, não pode ser concluída a partir das informações do banco

O método é incompleto no sentido em que não pode ser aplicado quando, no esquema do banco de dados, temos dois atributos com o mesmo domínio. Além disso, não é feito nenhum paralelo com a álbebra relacional nem análise da complexidade computacional do algoritmo para construção de modelos.

[LL86] N.Lerat and W.Lipski. Nonapplicable nulls. Theoretical Computer Science, 46,1986, pp.67-82.

Define o conceito de vazios não aplicável - aquele que não é desconhecido, mas sabe-se que não existe. Este tipo de valor vazio, que muitas vezes é visto como falha de projeto, é, em muitos casos, uma forma mais natural de se representar a realidade. Isto justifica o estudo desse tipo de valor vazio e a busca de um modelo formal para representá-lo. Define-se o conceito de "instância universal legal" - que é, basicamente, uma tabela especial onde é permitido o uso dos vazios inaplicáveis. É apresentado um método para transformar essa tabela em um conjunto de relações sem vazios, equivalentes, semanticamente, à mesma. São trabalhados, também, métodos para transformar e avaliar acessos a essas instâncias universais legais: através da criação de uma linguagem de acesso própria, as consultas são "traduzidas" para consultas normais. A definição dessa linguagem visa criar uma interface mais agradável para o usuário final.

[Lip79] W.Lipski. On semantic issues connected with incomplete information databases. ACM Transactions on Database Systems, 4(3), September 1979, pp.262-296. 
Propõe um modelo formal, chamado Sistemas de Informação para descrever um banco de dados com informação incompleta. São apresentados os conceitos de interpretação externa (mundo real) e interna (banco de dados) de uma consulta. Em relação a consultas externas, colocam-se dois níveis para uma dada consulta $Q$ : $\|\mathrm{Q}\|_{*}$, as informações concluídas, com certeza, a partir do banco de dados, e $\|\mathrm{Q}\|^{*}$, as informações que são possíveis de serem concluídas a partir dos dados no banco. Constrói-se um algoritmo para o cálculo desses dois níveis.

[LS90] K.C.Liu and R.Sunderraman. Indefinite and maybe information in relational databases. ACM Transactions on Database Systems, 15(1), March 1990, pp.1-39.

É introduzida uma estrutura, a I-tabela, que estende as tabelas do modelo relacional, para tratar disjunções e informações incertas. São apresentados os conceitos básicos de representação da I-tabela, em termos de possibilidades e incertezas, e redundância nessas estruturas. Define-se também o conceito de correção em termos de representações das tabelas, e os operadores da álgebra relacional são estendidos de maneira provadamente correta.

[LS91] K.C.Liu and R.Sunderraman. A generalized relational model for indefinite and maybe information. IEEE Transactions on Knowledge and Data Engineering, 1(3), March 1991, pp.65-77.

É definida uma estrutura, a M-tabela, que é, basicamente uma extensão das Itabelas definidas no artigo anterior, que permite incorporar fatos disjuntivos de relações diferentes. Os mesmos conceitos de representação, redundância e correção dos operadores são definidos para as novas estruturas, e os operadores relacionais são estendidos corretamente. Além desses, são apresentados dois novos operadores específicos para M-tabelas: a R-projeção e a Intercalação.

[Mai83] D.Maier. The theory of relational databases. Computer Science Press, Rockville, 1983.

Apresenta detalhadamente os conceitos da teoria de bancos de dados relacionais, entre eles: relações, operadores relacionais, dependências, normalização. Dedica um capítulo a valores vazios, informação parcial e semântica de bancos de dados.

[Min82] J.Minker. On indefinite databases and the Closed World Assumption. In Proceedings of the 6th Conference on Automated Deduction, D.W.Loveland, Ed., Lectures Notes in Computer Science, 138, Springer-Verlag, New York, June 1982, pp.292-308.

$\mathrm{O}$ artigo estende o conceito de CWA - hipótese do mundo fechado para a GCWA - hipótese do mundo fechado generalizado, para resolver problemas que aparecem em bancos de dados indefinidos, quando se assume a CWA - nesses casos, a CWA 
leva a inconsistência no banco. Basicamente, a GCWA só permite assumir um fato negativo quando o mesmo 1)não pode ser concluído a partir do banco de dados, como na CWA e 2)o fato concluído não leva a inconsistências no banco. A GCWA é compatível com bancos de dados com valores vazios tipo "desconhecido, mas aplicável e é um dos valores que já aparece no banco".

[MUV84] D.Maier, U.Ullman and M.Vardi. On the foundations of the Universal Relation Model. ACM Transactions on Database Systems, 9(2), June 1984, pp.283-308.

Apresenta o conceito de relação universal, e os objetivos da criação desse modelo - basicamente a facilidade de uso, liberando o usuário da tarefa de descrever as navegações entre as relações. Descreve também as principais hipóteses utilizadas na definição do modelo.

[Rei78] R.Reiter. On closed world databases. In Logic and Data bases, H.Gallaire and J.Minker, Eds., Plenum Press, New York, 1978, pp.55-76.

Introduz os conceitos formais de CWA (hipótese do mundo fechado) e OWA(hipótese do mundo aberto) para bancos de dados. Basicamente, a CWA afirma que todo fato positivo não representado pode ser assumido como falso. Isso significa que se tem o conhecimento absoluto dos fatos, e portanto, não permite a representação de fatos indefinidos. A OWA afirma o contrário, isto é, nada pode ser assumido a partir de um fato que não se conhece. É apresentado um método eficiente de avaliação de consultas sob a CWA. As consultas são decompostas até se alcançar uma subconsulta básica, cuja avaliação é equivalente na CWA e na OWA. Comenta que a CWA pode levar a inconsistências, e é apresentada uma condição suficiente para que o banco seja consistente.

[Rei84] R.Reiter. Towards a logical reconstruction of relational database theory. In On Conceptual Modeling, M.Brodie, J.Mylopoulos, and J.Schmidt, Eds. SpringerVerlag, Berlin, 1984, pp.191-238.

O texto descreve formas de representação da teoria de bancos de dados relacionais via teoria de prova em lógica de primeira ordem, abrangendo definições de relações, de restrições de integridade e de consultas. Faz uma comparação com a descrição do modelo relacional através de teoria de modelos da lógica. O objetivo é mostrar que a a teoria de prova é mais abstrata e pode incorporar mais facilmente outros aspectos como informação incompleta, valores vazios e hierarquias.

[Rei86] R.Reiter. A sound and sometimes complete query evaluation algorithm for relational databases with null values. In Journal of ACM,33(2), April 1986, pp.349370. 
Descreve, brevemente, a forma de reconstruir a teoria de bancos de dados relacionais através da teoria de prova em lógica de primeira ordem. É então apresentada uma linguagem de consulta, baseada também em lógica de primeira ordem e um algoritmo para decomposição de uma consulta expressa nessa linguagem, para avaliação da mesma. Nesse algoritmo, sacrifica-se a completude em alguns casos para diminuir o custo computacional. Nos casos de consultas conjuntivas universalmente quantificadas, consultas positivas e consultas a bancos de dados sem valores vazios, o algoritmo é completo.

[RKS89] M.A.Roth, H.F.Korth, and A.Silberschatz. Null values in nested relational databases ACTA informatica, 26, 1989, pp.615-642.

Estendem os conceitos de valores vazios para bancos de dados não normalizados. Utilizam os valores não-aplicável, aplicável e sem informação, como nos bancos normalizados. Um caso especial de valores vazios - o conjunto vazio - é analisado. São revistos também os conceitos de tuplas e relações mais informativos. Os operadores de normalização e não-normalização, bem como alguns operadores da álgebra relacional são estendidos para incorporar valores vazios. Consideram-se relações de igualdade apenas entre os valores vazios não aplicáveis, e entre valores vazios identificados. São comentados também os efeitos do uso de valores vazios nas regras de dependências no banco de dados.

[Set89] V.W.Setzer. Bancos de Dados. Edgard Blücher Ltda, São Paulo, 1989.

Descreve conceitos gerais de bancos de dados, como modelos de dados, projeto lógico e projeto físico, e gerenciadores. Propõe uma forma de implementação para um gerenciador de bancos de dados que permite o uso de relações não normalizadas.

[Sik81] L.Siklossy. Efficient query evaluation in relational data bases with missing values. In Information Processing Letters, 13(4,5), 1981, pp.160-163.

Aponta o problema da avaliação incorreta de consultas na lógica de 3 valores proposta por Codd. Apresenta uma algoritmo eficiente para consultas que possam ser expressas em lógica sentencial, e onde cada átomo é da forma (atributo=valor). $\mathrm{O}$ algoritmo sacrifica as consultas em lógica de primeira ordem em função da complexidade.

[Var86] M.Y.Vardi. On the integrity of databases with incomplete information. Proceedings of the 5o. ACM SIGACT-SIGMOD Symposium on Principles of Database systems, 1986, pp.252-266.

$\mathrm{O}$ artigo mostra a complexidade da verificação de integridade em bancos de dados com informações incompletas, isto é, verificação de satisfabilidade de dependências. A complexidade computacional da verificação de integridade é, sob a OWA, de 
tempo polinomial, e, sob a CWA, tempo exponencial. Além disso, verifica-se que tipo de lógica é necessária para axiomatizar finitamente este problema. Sob a OWA, necessita-se de lógica de pontos fixos, e, sob a CWA, é necessário lógica de segunda ordem existencial. Para a avaliação de consultas, a dificuldade computacional se inverte: sob a CWA, a complexidade é a mesma que em bancos sem informação incompleta, e, sob a OWA, o problema é intratável.

[Win88] M.W.Winslett. A model-based approach to updating databases with incomplete information. ACM Transactions on Database Systems, 13(2), 1988, pp.167196.

Aborda aspectos da atualização em bancos de dados incompletos através da formalização em teoria de prova da lógica de primeira ordem. Propõe uma linguagem para atualização, discorre sobre a semântica dessa linguagem através da teoria de modelos e desenvolve um algoritmo para interpretar essa linguagem de forma correta em relação à semântica apresentada.

[YC88] L.Y.Yuan and D.Chiang. A sound and complete query evaluation algorithm for relational databases with null values. SIGMOD Record, 17(3), September 1988, pp.74-81.

$\mathrm{O}$ artigo estende o conceito de relação para agrupar disjunções - uma tupla é agora $\mathrm{t}=t_{1} \vee t_{2} \vee \ldots \vee t_{n}$. Estende também a álgebra relacional para lidar com as relações estendidas. Desenvolve um algoritmo para avaliação de consultas, nos mesmos moldes de Reiter (1986). Porém, o algoritmo pode devolver relações estendidas, e isto permite que o mesmo seja correto e completo. Para conseguir a completude, que não havia no algoritmo de Reiter, foi necessário aumentar a complexidade do mesmo, mas em alguns casos, o algoritmo mantém a complexidade menor e mesmo assim consegue ser completo - por exemplo, em consultas com o quantificador existencial.

[Yue91] K.Yue. A more general model for handling missing information in relational databases using a 3-valued logic. SIGMOD Record, 20(3), September 1991, pp.4349.

O artigo propõe uma simplificação na lógica de 7 valores, necessária para a representação dos valores vazios: inaplicável, aplicável e sem informação (pode ser inaplicável ou aplicável). A idéia principal é igualar o valor Inaplicável ao Falso. São apresentadas funções lógicas para recuperar, posteriormente, o Inaplicável. O autor descarta o uso da lógica de 4 valores porque não há, segundo ele, uma simplificação razoável da lógica de 7 valores, que captura a generalidade dos 3 tipos de vazios, para a lógica de 4 valores. 
[Zan84] C.Zaniolo. Database relations with null values. Journal of Computer and System Sciences, 28, 1984, pp.142-166.

O texto inicia apontando algumas falhas da extensão feita por Codd para a álgebra relacional com vazios - tal extensão não preserva algumas propriedades básicas da teoria de conjuntos como $\mathrm{S}=\mathrm{S}, \mathrm{S} \cap \mathrm{T} \subseteq \mathrm{S}, \mathrm{S} \cup \mathrm{T} \supseteq \mathrm{S}$. É ressaltada a dificuldade de se trabalhar com os vazios "aplicável" e "inaplicável", e propõe-se o vazio mais geral "não há informação" Através da definição deste e da lógica de 3 valores, desenvolve-se uma teoria simples que conserva as propriedades perdidas por Codd, com base nos conceitos de "tupla mais informativa", "relações mais informativas" e "relações estendidas" (x-relações).

[Zic90] Ziccari, R. Incomplete information in Object-oriented databases. SIGMOD Record, 19(3), September 1990.

$\mathrm{O}$ artigo apresenta os tipos de informação incompleta que podem existir num banco de dados orientado a objetos: dados incompletos na definição das classes ou nos valores dos objetos. Para o primeiro caso, mostra-se uma solução tipo "classe genérica", que não tem nenhum tipo de valor associado. Assim, ao ser criado um objeto nessa classe, só é fornecido o nome do mesmo. Posteriormente, esta classe deve poder ser atualizada para se completar as informações a seu respeito. Os objetos dessa classe poderão então ser alterados, para se completarem suas informações. $\mathrm{O}$ outro caso de informação incompleta é a falta de valores em alguns atributos de um objeto. Este caso é bem parecido com o problema de valores vazios em bancos de dados não normalizados, pois permitem-se conjuntos de atributos numa mesma classe. O tratamento deve ser feito de maneira similar.

[ZP92] E.Zimányi and A.Pirotte. Imperfect Knowledge in databases. Research Report RR92-36, Université Catholique de Louvain, October 1992.

Descreve diversas formas de informação incompleta em bancos de dados, tais como: valores vazios de vários tipos, disjunções, informações possíveis, probabilidades, informação difusa (fuzzy), e exceções. 